\title{
A combined oscillation cycle involving self-excited thermo-acoustic and hydrodynamic instability mechanisms
}

D. Fredrich, ${ }^{\text {a) }}$ W. P. Jones, and A. J. Marquis

Department of Mechanical Engineering, Imperial College London, Exhibition Road, London SW7 2AZ, United Kingdom

(Dated: 12 July 2021)

The paper examines the combined effects of several interacting thermo-acoustic and hydrodynamic instability mechanisms that are known to influence self-excited combustion instabilities often encountered in the late design stages of modern lowemission gas turbine combustors. A compressible large eddy simulation approach is presented, comprising the flame burning regime independent, modelled probability density function evolution equation/stochastic fields solution method. The approach is subsequently applied to the PRECCINSTA (PREdiction and Control of Combustion INSTAbilities) model combustor and successfully captures a fully self-excited limit-cycle oscillation without external forcing. The predicted frequency and amplitude of the dominant thermo-acoustic mode and its first harmonic are found to be in excellent agreement with available experimental data. Analysis of the phaseresolved and phase-averaged fields leads to a detailed description of the superimposed mass flow rate and equivalence ratio fluctuations underlying the governing feedback loop. The prevailing thermo-acoustic cycle features regular flame lift-off and flashback events in combination with a flame angle oscillation, as well as multiple hydrodynamic phenomena, i.e. toroidal vortex shedding and a precessing vortex core. The periodic excitation and suppression of these hydrodynamic phenomena is confirmed via spectral proper orthogonal decomposition and shown to be controlled by an oscillation of the instantaneous swirl number. Their local impact on the heat release rate, which is predominantly modulated by flame-vortex roll-up and enhanced mixing of fuel and oxidiser, is further described and investigated. Finally, the temporal relationship between the flame 'surface area', flame-averaged mixture fraction and global heat release rate are found to be directly correlated.

a)Corresponding author d.fredrich15@imperial.ac.uk 


\section{INTRODUCTION}

Modern low-emission aero-engine and stationary gas turbine combustors typically operate under lean, partially premixed conditions and employ swirling flows for flame stabilisation purposes. However, despite being highly effective in the reduction of environmentally harmful pollutant emissions, such state-of-the-art combustor configurations are often prone to the onset of undesirable combustion dynamics as a result of self-excited thermo-acoustic and hydrodynamic instabilities ${ }^{1,2}$. These instabilities, more generally known as combustion instabilities, are characterised by high-amplitude oscillations of the pressure and heat release rate and arise when a resonant feedback loop between the combustion chamber acoustics and the unsteady reactive flow field is established. The time and volume integral of the pressure times the heat release rate must therefore be greater than zero in order to satisfy the Rayleigh criterion ${ }^{3}$. If this is satisfied, an increase of the oscillation amplitude will occur until the acoustic energy added to the system equals its energy losses. The ensuing state of zero growth rate, commonly referred to as limit-cycle oscillation, depends upon a variety of complex, highly non-linear physical mechanisms.

One of the key parts of the feedback loop for thermo-acoustic instabilities in partially premixed combustion systems are equivalence ratio oscillations. This particular feedback mechanism was first described by Lieuwen \& $\mathrm{Zinn}^{4}$ and stems from a fluctuating supply of fresh reactants into the main flame zone, generating unsteady heat release by modulating the reaction rate per unit flame 'area'. The resulting pressure disturbance at the flame front propagates up- and downstream in the form of an acoustic wave, where it can perturb the fuel and oxidiser supply rates. Different acoustic impedances of the respective inflow streams may then periodically vary the mixture's local equivalence ratio closing the feedback loop. A convective time delay between the point of fuel injection and the flame front can be introduced, governing the phase lag between the pressure and heat release rate fluctuations ${ }^{5}$. The exact role of equivalence ratio oscillations in sustaining thermo-acoustic instabilities has since been studied extensively, e.g. in Refs. ${ }^{6-9}$.

A second major category of physical phenomena contributing to the generation of unsteady heat release in swirling flames are hydrodynamic instabilities, which have recently been reviewed by Candel et al. ${ }^{10}$. These can be linked to two different types of periodic mechanisms: $(i)$ perturbations of the turbulent burning velocity due to flame angle oscil- 
lations induced by temporal fluctuations of the swirl number ${ }^{5,11-13}$, and (ii) modulation of the flame's 'surface area' via the interaction of large-scale coherent structures, i.e. toroidal vortex shedding ${ }^{14-17}$ or precessing vortex cores $(\mathrm{PVC})^{18-22}$, with the turbulent flame front. The latter can also play an important role in flame lift-off ${ }^{23}$ and flashback ${ }^{24}$ events, and influence the mixing process between fuel and oxidiser ${ }^{25}$.

Large eddy simulation (LES) is a powerful method potentially capable of facilitating the prevention of both thermo-acoustic and hydrodynamic instabilities in gas turbines. In LES, governing transport equations describing the reactive flow field are spatially filtered and closed through turbulence and combustion models accounting for the sub-grid scale (sgs) contributions. A detailed review on numerical prediction and control methods for combustion instabilities can be found in Dowling \& Morgans ${ }^{26}$, Huang \& Yang ${ }^{27}$ and Poinsot ${ }^{28}$. Generally, two different modelling strategies exist. In the first approach, so-called open-loop - or coupled - LES methods, conventional incompressible LES is combined with low-order modelling techniques from acoustic theory, see e.g. ${ }^{29-33}$. The frequency and amplitude of potential limit-cycle oscillations in any combustor geometry can thereby be systematically determined. For this purpose, a system's heat release response to incoming velocity fluctuations is obtained in LES by externally forcing the inflow velocity at different frequencies and amplitudes. The resulting flame transfer function (FTF) for linear analysis ${ }^{34}$ or flame describing function (FDF) for weakly non-linear analysis ${ }^{35}$ is then evaluated with a low-order acoustic network model or alternatively a Helmholtz solver providing the corresponding growth rates and frequencies for each forcing amplitude. As an alternative, a fully compressible LES formulation, see e.g. ${ }^{36}$, can be utilised to provide a description of self-excited thermo-acoustic phenomena, thus allowing the propagation of acoustic waves represented by temporal fluctuations in the pressure field. In this second approach, account is taken of the inherent coupling mechanisms between the flame, flow and acoustics inside a combustion chamber. Such unforced LES computations often prove challenging, however, as they require careful consideration of all geometrical features making up the domain as well as accurate boundary conditions including acoustic impedances at all inlets and outlets. Hence, there are only a very limited number of computational studies available in the open literature utilising compressible LES to study self-excited combustion instabilities in gas turbine combustors $^{37-47}$.

The PRECCINSTA (PREdiction and Control of Combustion INSTAbilities) model 
combustor investigated in the present work involves a partially premixed, swirl-stabilised methane-air flame and was derived from a low-emission industrial design by Turbomeca. The test case was first set-up and investigated by Meier et al. ${ }^{48}$ in an experimental measurement campaign at the German Aerospace Center (DLR). It was shown to exhibit a strong flame oscillation driven by thermo-acoustic instabilities at a global equivalence ratio of $\phi_{\text {glob }}$ $=0.7$ and thermal power of $P_{t h}=25.1 \mathrm{~kW}$ - labelled oscillating flame case (1). A number of follow-up studies at various operating conditions have since been carried out attempting to identify the underlying mechanisms involved in the flame oscillation. Among the different phenomena studied in these works are the role of mass flow rate and equivalence ratio oscillations $^{9,48}$, the dynamics of coherent vortex structures, i.e. the $\mathrm{PVC}^{49-52}$ and symmetric vortex shedding ${ }^{50}$, as well as the transition between bi-stable flame shapes ${ }^{53-57}$. It is important to note that each of these studies only focused on one specific feedback mechanism at a time. Hydrodynamic instability modes, for example, were typically examined in a perfectly premixed test case set-up, in order to prevent equivalence ratio oscillations from developing.

Previous numerical studies have mainly targeted the PRECCINSTA test case to validate specific LES combustion models based on its reference operating condition, case (2a) with $\phi_{\text {glob }}=0.83$ and $P_{t h}=30 \mathrm{~kW}$, featuring a 'stable' flame ${ }^{48}$. Only very few studies have attempted to investigate the experimentally observed oscillating flame behaviour and its underlying feedback mechanisms: Roux et al. ${ }^{38}$ computed both a non-reacting flow and a reacting flow involving a subtly pulsating flame - case $(2 \mathrm{~b})$ at $\phi_{\text {glob }}=0.75$ and $P_{t h}=27 \mathrm{~kW}$ using a compressible LES method in combination with acoustic analysis. Comparisons with experimental data revealed good agreement for the predicted acoustic and hydrodynamic modes in the cold flow as well as for the thermo-acoustic mode obtained with combustion. The oscillating flame case (1) was first simulated by Franzelli et al. ${ }^{41}$. Their compressible LES successfully reproduced self-excited oscillations at a frequency of $390 \mathrm{~Hz}$ - about $100 \mathrm{~Hz}$ above the experimentally measured value. The overestimation was argued to be the result of an inaccurately modelled acoustic impedance at the fuel injection inlets. It was furthermore concluded that resolving the fuel-air mixing, as opposed to prescribing a fully premixed mixture at the domain inlet, is crucial for the prediction of the thermo-acoustic coupling in this test case. Lourier et al. $^{43}$ later performed a scale-adaptive simulation (SAS) of the oscillating flame case as well as additional experimental measurements with adjusted acoustic boundary conditions. For this purpose, a choked orifice plate $15 \mathrm{~cm}$ upstream of 
the air plenum was equipped inside the inflow nozzle creating a well-defined acoustic inlet boundary. This however, led to a marginal decrease of the predominant thermo-acoustic frequency by about $15 \mathrm{~Hz}$. Moreover, rigid metal walls were securely mounted in between the combustion chamber corner posts to mitigate the effects of acoustic damping, which was evident in the initial measurements by Meier et al. ${ }^{48}$ due to the use of loosely-fitted quartz glass windows. Power spectral density (PSD) analysis of the air plenum and combustion chamber pressure signals accordingly provided an increase in the oscillation amplitude of around $10 \mathrm{~dB}$ with the adjusted set-up. The employed SAS model switched between an LES and an unsteady Reynolds-averaged Navier-Stokes (URANS) solution depending on the local grid resolution. Good overall agreement was obtained for the thermo-acoustic frequency and its amplitude, showing only a relatively small over-prediction of about 15$25 \mathrm{~Hz}$ and $5 \mathrm{~dB}$, respectively. It is to be noted that neither of these prior studies identified any hydrodynamic modes in the reacting flow case.

Despite considerable (mostly experimental) research efforts in the past, the aforementioned thermo-acoustic and hydrodynamic instability phenomena are still not fully understood and are typically examined individually, with the purpose of isolating the various feedback mechanisms at play. This limited understanding of the non-linear dynamics, interactions and coupling of multiple instability mechanisms presents a significant knowledge gap in the field of combustion. The objective of the current work is to build upon and contribute to the existing knowledge by: $(i)$ presenting a LES method capable of capturing several interacting non-linear thermo-acoustic and hydrodynamic feedback mechanisms, and (ii) reporting and studying a newly identified combined limit-cycle oscillation involving such mechanisms. The former may ultimately provide a means for facilitating the prevention of combustion instabilities in the early design stages of novel gas turbine combustors. The latter includes a first-of-its-kind comparison between experimentally and numerically obtained phase-averaged radial profiles, as well as the quantification of the instantaneous swirl number and a novel description of its role in the generation of coherent vortex structures.

\section{MATHEMATICAL FORMULATION AND NUMERICAL METHOD}

The LES solver employed in the present work comprises a transported probability density function $(p d f)$ approach, which is solved by the stochastic fields method to account for 
turbulence-chemistry interaction. The method does not involve any explicit assumptions of burning regime and is equally applicable to non-premixed, partially premixed and perfectly premixed turbulent flames, see e.g. ${ }^{58-60}$. A similar compressible LES- $(p d f)$ approach was also recently derived by Pant et al. ${ }^{61}$ and two previous works have applied such methods in the context of supersonic flows ${ }^{62,63}$.

\section{A. Filtered transport equations}

Application of a density-weighted LES filtering operation to the governing equations of motion and scalar transport results in:

$$
\begin{gathered}
\frac{\partial \bar{\rho}}{\partial t}+\frac{\partial \bar{\rho} \widetilde{u}_{i}}{\partial x_{i}}=0 \\
\frac{\partial \bar{\rho} \widetilde{u}_{i}}{\partial t}+\frac{\partial \bar{\rho} \widetilde{u}_{i} \widetilde{u}_{j}}{\partial x_{i}}=-\frac{\partial \bar{p}}{\partial x_{j}}+\frac{\partial}{\partial x_{i}} \mu\left[\left(\frac{\partial \bar{u}_{i}}{\partial x_{j}}+\frac{\partial \bar{u}_{j}}{\partial x_{i}}\right)-\frac{2}{3} \frac{\partial \bar{u}_{k}}{\partial x_{k}} \delta_{i j}\right]-\frac{\partial \tau_{i j}^{s s s}}{\partial x_{i}} \\
\frac{\partial \bar{\rho} \widetilde{n}_{\alpha}}{\partial t}+\frac{\partial \bar{\rho} \widetilde{u}_{i} \widetilde{n}_{\alpha}}{\partial x_{i}}=\overline{\rho \dot{\omega}}-\frac{\partial}{\partial x_{i}}\left[\bar{J}_{\alpha, i}+\bar{\rho}\left(\widetilde{u_{i} n_{\alpha}}-\widetilde{u}_{i} \widetilde{n}_{\alpha}\right)\right] \\
\frac{\partial \bar{\rho} \widetilde{h}_{t}}{\partial t}+\frac{\partial \bar{\rho} \widetilde{u}_{i} \widetilde{h}_{t}}{\partial x_{i}}=\frac{\partial \bar{p}}{\partial t}-\frac{\partial}{\partial x_{i}}\left[\bar{J}_{h, i}+\bar{\rho}\left(\widetilde{u_{i} h_{t}}-\widetilde{u}_{i} \widetilde{h}_{t}\right)\right]
\end{gathered}
$$

where $\rho$ represents the density, $u_{i}$ and $x_{i}$ are, respectively, the velocity and spatial coordinate in $i$-direction, $t$ is the time, $p$ the pressure, $\mu$ the viscosity and $\delta_{i j}$ denotes the Kronecker delta. The deviatoric part of the sgs stress tensor $\tau_{i j}^{s g s}=\bar{\rho}\left(\widetilde{u_{i} u_{j}}-\widetilde{u_{i}} \widetilde{u}_{j}\right)$ is determined with the Smagorinsky model ${ }^{64}$ :

$$
\tau_{i j}^{s g s}-\frac{\delta_{i j}}{3} \tau_{k k}^{s g s}=-2 \mu_{s g s}\left(\widetilde{e}_{i j}-\widetilde{e}_{k k} \frac{\delta_{i j}}{3}\right)
$$

using the sgs viscosity:

$$
\mu_{s g s}=\bar{\rho}\left(C_{S} \Delta\right)^{2}\left\|\widetilde{e}_{i j}\right\|
$$

where $\widetilde{e}_{i j}$ is the resolved rate of strain tensor and $\left\|\widetilde{e}_{i j}\right\| \equiv\left(2 \widetilde{e}_{i j} \widetilde{e}_{i j}\right)^{1 / 2}$ is its Frobenius norm. The filter width $\Delta$ is taken as the cube root of the local grid cell volume and the parameter $C_{S}$ is obtained through the dynamic procedure of Piomelli \& Liu ${ }^{65}$, while the isotropic parts of the viscous and sgs stresses are absorbed into the pressure. Eq. (3) represents the filtered conservation equation for the specific mole number of each of the $N_{s}$ chemical species, $n_{\alpha}=\left[n_{1}, \ldots, n_{N_{s}}\right]$ and where $\overline{\rho \dot{\omega}_{\alpha}}$ is the net formation rate per unit volume of species $\alpha$ through chemical reaction. The energy equation (4) is written in terms of the total enthalpy 
$h_{t}=h+\frac{1}{2} u_{i} u_{i}$, so that there are $N_{s}+1$ scalar conservation equations. Radiative heat loss has been neglected in the enthalpy equation. Fourier and Fick's law of diffusion are utilised for the heat and species diffusion fluxes, $J_{h, j}$ and $J_{\alpha, j}$, together with the assumptions of equal diffusivities for all chemical species and unity Lewis number ${ }^{66}$ resulting in:

$$
J_{h, i}=-\frac{\mu}{\sigma} \frac{\partial h}{\partial x_{i}}, \quad J_{\alpha, i}=-\frac{\mu}{\sigma} \frac{\partial n_{\alpha}}{\partial x_{i}}
$$

respectively, where $\sigma$ is the Prandtl/Schmidt number. However, due to the difficulties encountered in evaluating the filtered values of the chemical source terms, the combined scalar transport equations (3) and (4) are not solved directly in the present work.

\section{B. Probability density function approach}

Following Gao \& O'Brien ${ }^{67}$, an evolution equation for the spatially filtered joint finegrained $p d f$ is introduced - here termed the sgs-pdf - to account for the influence of $s g s$ fluctuations on the species formation rates. Thus, $\widetilde{P}_{s g s}$ represents the one-point joint $p d f$ for each of the $N_{s}$ scalars representing the specific mole number of each species and the enthalpy. If the unknown terms are modelled following the procedure of Brauner et al. ${ }^{68}$, the sgs-pdf equation appears in the closed form:

$$
\begin{array}{r}
\bar{\rho} \frac{\partial \widetilde{P}_{s g s}(\underline{\psi})}{\partial t}+\bar{\rho} \widetilde{u}_{i} \frac{\partial \widetilde{P}_{s g s}(\underline{\psi})}{\partial x_{i}}+\sum_{\alpha=1}^{N_{s}+1} \frac{\partial J_{i, \alpha}(\underline{\bar{\phi}})}{\partial x_{i}} \frac{\partial \bar{P}_{s g s}(\underline{\psi})}{\partial \psi_{\alpha}} \\
+\sum_{\alpha=1}^{N_{s}+1} \frac{\partial}{\partial \psi_{\alpha}}\left[\bar{\rho} \dot{\omega}_{\alpha}(\underline{\psi}) \widetilde{P}_{s g s}(\underline{\psi})\right] \\
=\frac{\partial}{\partial x_{i}}\left[\left(\frac{\mu_{s g s}}{\sigma_{s g s}}\right) \frac{\partial \widetilde{P}_{s g s}(\underline{\psi})}{\partial x_{i}}\right] \\
-\frac{C_{d}}{2 \tau_{s g s}} \sum_{\alpha=1}^{N_{s}+1} \frac{\partial}{\partial \psi_{\alpha}}\left[\bar{\rho}\left(\psi_{\alpha}-\widetilde{\phi}_{\alpha}(\mathbf{x}, t)\right) \widetilde{P}_{s g s}(\underline{\psi})\right]
\end{array}
$$

where $J_{j, \alpha}$ is given by Eq. 5 , as appropriate. $\psi_{\alpha}$ denotes the sample space for the scalar $\phi_{\alpha}$ with $1 \leq \alpha \leq N_{s}+1$. The terms on the left-hand side of Eq. (6), including $\dot{\omega}_{\alpha}$, which for chemical species includes the reaction rate source and for the total enthalpy the pressure derivative $\partial p / \partial t$, appear in closed form and do not require modelling - this being the main advantage of the $p d f$ formulation. In contrast, the terms on the right-hand side of Eq. (6) are modelled, representing turbulent transport of the $p d f$ and sgs-mixing due to the action 
of viscosity. Both terms are identically zero in the event that the sgs variations are zero. For turbulent transport, a gradient closure directly analogous to the Smagorinsky model has been adopted, while the linear mean square estimation (LMSE) closure ${ }^{69}$ has been applied for sgs-mixing. The sgs-mixing time scale is given by $\tau_{s g s}=\bar{\rho} \Delta^{2} / \mu_{s g s}$ and the $s g s$ Prandtl/Schmidt number $\sigma_{s g s}$ and $s g s$-mixing constant $C_{d}$ are assigned the values 0.7 and 2.0 , respectively, ${ }^{70}$.

\section{Stochastic fields method}

It is not feasible to numerically solve the $p d f$ equation (6) directly, due to the large number of independent variables, but also because $\widetilde{P}_{s g s}(\underline{\psi})$ is a generalised function, defined only in terms of its integral properties. As a consequence, a stochastic solution method is adopted instead, where the $p d f$ equation is represented by an ensemble of stochastic fields ${ }^{71}$, corresponding to a system of stochastic differential equations $(s d e s)^{72}$ equivalent to Eq. (6). The stochastic fields method has been previously applied in LES, e.g. by Jones \& Navarro-Martinez ${ }^{73}$, with the fields $\xi_{\alpha}^{n}(\mathbf{x}, t)$ being given by:

$$
\begin{array}{r}
\bar{\rho} \mathrm{d} \xi_{\alpha}^{n}+\bar{\rho} \widetilde{u}_{i} \frac{\partial \xi_{\alpha}^{n}}{\partial x_{i}} \mathrm{~d} t-\bar{\rho} \dot{\omega}_{\alpha}^{n}\left(\underline{\xi}^{n}\right) \mathrm{d} t+\frac{\partial J_{i, \alpha}^{n}}{\partial x_{i}} \mathrm{~d} t=\frac{\partial}{\partial x_{i}}\left(\frac{\mu_{s g s}}{\sigma_{s g s}} \frac{\partial \xi_{\alpha}^{n}}{\partial x_{i}}\right) \mathrm{d} t \\
-\frac{\bar{\rho} C_{d}}{2 \tau_{s g s}}\left(\xi_{\alpha}^{n}-\widetilde{\phi}_{\alpha}\right) \mathrm{d} t+\left(2 \bar{\rho} \frac{\mu_{s g s}}{\sigma_{s g s}}\right)^{1 / 2} \frac{\partial \xi_{\alpha}^{n}}{\partial x_{i}} \mathrm{~d} W_{i}^{n}(t)
\end{array}
$$

where $1 \leqslant n \leqslant N$, with $N=8$ being the number of fields ${ }^{73}$, and $1 \leqslant \alpha \leqslant N_{s}+1$. The Wiener process $\mathrm{d} W_{i}^{n}(t)$, different for each field but independent of the spatial location $\mathbf{x}$, is approximated by time-step increments $\eta_{i}^{n}(\mathrm{~d} t)^{1 / 2}$ containing the $\{-1,1\}$ dichotomic random vector $\eta_{i}^{n}$. The solutions of the stochastic fields equation (7) are continuous, differentiable and smooth on the scale of the filter width in space and continuous but not differentiable in time. Further details of the solution method can be found in Jones \& $\operatorname{Prasad}^{74}$. The filtered value of each scalar is finally obtained by averaging over the stochastic fields.

\section{Computational set-up}

The in-house, fully compressible, block-structured, parallel, boundary conforming coordinate LES code, BOFFIN-LESc has been used for the computations to be presented in the following sections. The code employs Cartesian velocity components with a co-located 


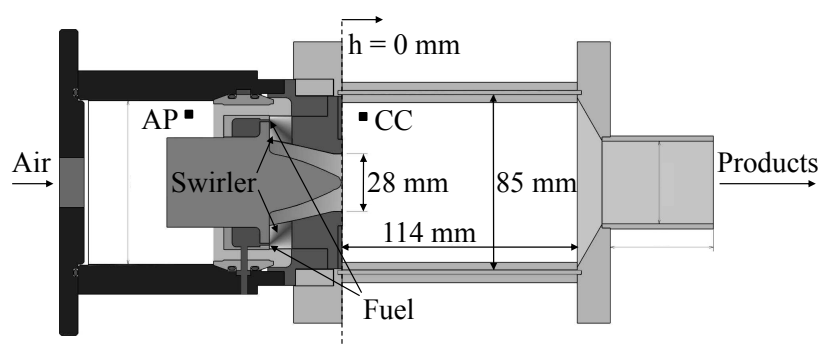

FIG. 1. Schematic combustor geometry indicating the locations of the pressure probes AP (air plenum) and CC (combustion chamber). The burner nozzle exit / combustion chamber entry plane is defined as $h=0 \mathrm{~mm}$.

variable storage arrangement. It is based on an implicit pressure based formulation and is second order accurate in space and time. All spatial derivatives are represented by standard central differences and the advancement in time is performed with a time-centered Crank-Nicholson scheme. Further details of the algorithm can be found in ${ }^{75}$. The stochastic fields equations are solved using an approximately factored Euler-Maruyama scheme ${ }^{76}$, details of which are given in Jones et al. ${ }^{70}$. For the chemistry, a 15-step / 19 species reduced mechanism for methane oxidation ${ }^{77}$ is included. An alternative and potentially less time consuming description of the reaction kinetics based on chemistry tabulation using artificial neural networks (ANNs) was recently presented by Readshaw et al. ${ }^{78}$. Standard non-reflective boundary conditions ${ }^{79,80}$ are implemented at the domain outlet to minimise pressure wave reflection. An approximate near wall model based on the semi-logarithm law of the wall ${ }^{81}$ is applied at all solid boundaries.

The solution domain comprises all of the main features of the test rig geometry shown in Fig. 1, including the upstream plenum and short downstream duct. The operating conditions of case (1) are applied with the air and fuel inlet velocities and density being fixed based on the respective mass flow rates, $\dot{m}_{\text {air }}=12.237 \mathrm{~g} / \mathrm{s}$ and $\dot{m}_{\text {fuel }}=0.5 \mathrm{~g} / \mathrm{s}$, together with a target temperature of $320 \mathrm{~K}$. Pure air is injected into the plenum through a large orifice, whereas pure methane is injected through a single hole in each of the 12 swirler vanes. A target pressure of 1 bar is specified at the domain outlet. The Kolmogorov length and time scales are estimated ${ }^{48}$ to be $0.1 \mathrm{~mm}$ and $35 \mu$ s respectively. Typical values of the turbulence intensities can be obtained from Fredrich et al. ${ }^{82}$. The computational mesh consisting of 2.7 
million grid points is refined in the combustion zone and fuel-air mixing regions ${ }^{82}$. Isothermal temperatures of $1400 \mathrm{~K}$ and $700 \mathrm{~K}$ are prescribed at the combustion chamber side and base plate walls, respectively, to include wall heat transfer ${ }^{55}$. Previous numerical studies ${ }^{82-84}$ have shown that an application of non-adiabatic combustion chamber walls noticeably improves the near-wall scalar fields and can induce local extinction of the outer flame base in line with experimental observations. None of the solver's default model parameters are adjusted in this work and no artificial forcing of the inlet velocities is imposed, to ensure that any potential oscillatory flow behaviour observed in the simulation is fully self-excited and selfsustained.

\section{RESULTS AND DISCUSSION}

After initialisation of the non-reacting flow field and its subsequent ignition via isothermal wall temperatures, a pronounced self-excited flame limit-cycle oscillation developed inside the combustor. A single period of this oscillation is visualised in Fig. 2 using iso-surfaces of the heat release rate (HRR) and fuel mass fraction. Eight different equally spaced phase angles $\left(P h_{1-8}\right)$ have been defined to enable analysis of the phase-resolved and phase-averaged fields throughout the rest of this work. The observed oscillation involves periodic flame liftoff and flashback events, as well as significant variations in the flame's 'surface area' and local mixture fraction (evaluated based on the carbon element). A similar flame behaviour was also reported in a recent experimental study, Temme et al. ${ }^{85}$ in which a swirl-stabilised, liquid fuel gas turbine combustor featured strong self-excited combustion instabilities driven by an equivalence ratio oscillation mechanism. While the potential impact of hydrodynamic phenomena was not explicitly investigated, the results did underline the importance of flame lift-off in these types of instabilities. In the present work, both thermo-acoustic and hydrodynamic instability mechanisms are identified. Their respective interactions and coupling are examined in the following sections, yielding a comprehensive description of the combined oscillation cycle. 

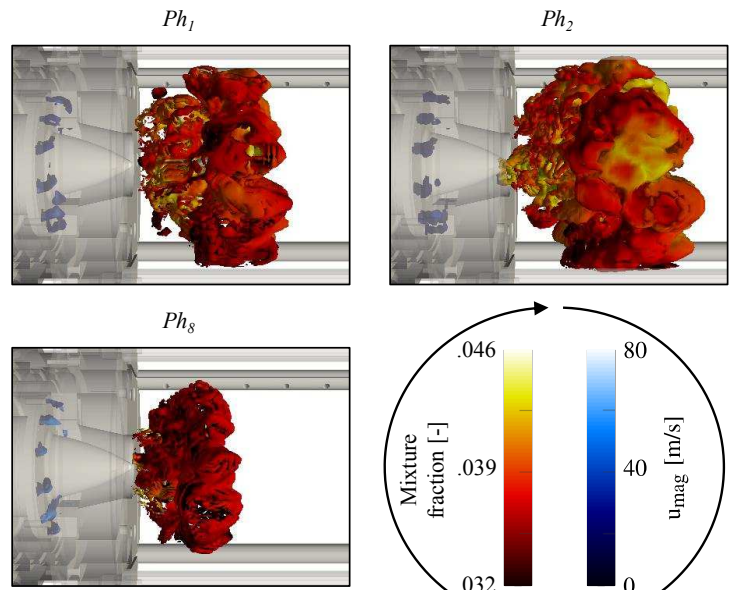

$\mathrm{Ph}_{7}$

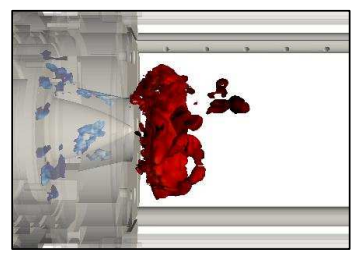

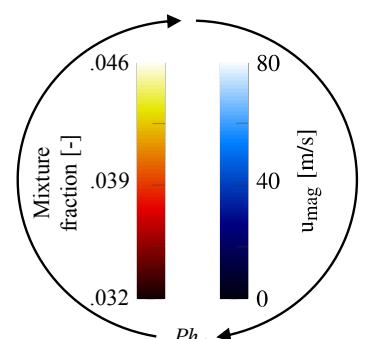

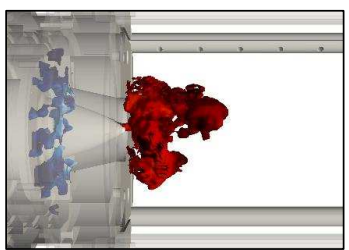

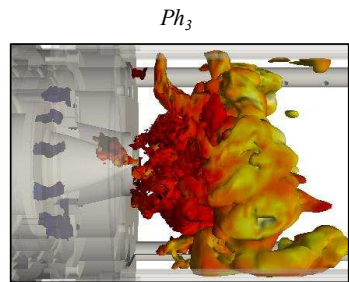

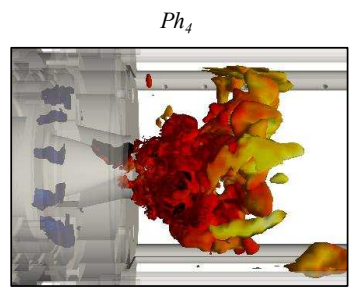

$\mathrm{Ph}_{5}$

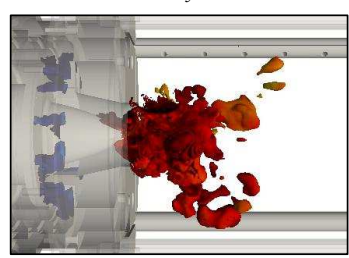

FIG. 2. Periodic evolution of the instantaneous flame topology and fuel injection represented by iso-surfaces of a constant local heat release rate of $150 \mathrm{MW} / \mathrm{m}^{3}$ and $\mathrm{CH}_{4}$ mass fraction of 0.1 , respectively. The former is coloured by mixture fraction, the latter by velocity magnitude.

\section{A. Limit-cycle quantification}

Figure 3 presents the fluctuation signals of the spatially integrated - or global - HRR and the local pressure recorded at probes AP (air plenum) and CC (combustion chamber). The HRR and chamber pressure both visibly oscillate in phase indicating a positive feedback loop, therefore globally satisfying the Rayleigh criterion $^{3}$ defined as:

$$
\int_{\Omega} \int_{T} p^{\prime} \dot{q}^{\prime} d \Omega d t>0
$$

where $p^{\prime}$ and $\dot{q}^{\prime}$ are the local pressure and HRR, $\Omega$ is the flow domain and $T$ denotes the oscillation period. Constant mean amplitudes further reveal a state of zero growth rate - typical of a limit-cycle oscillation. The existing modes are retrieved via PSD analysis 

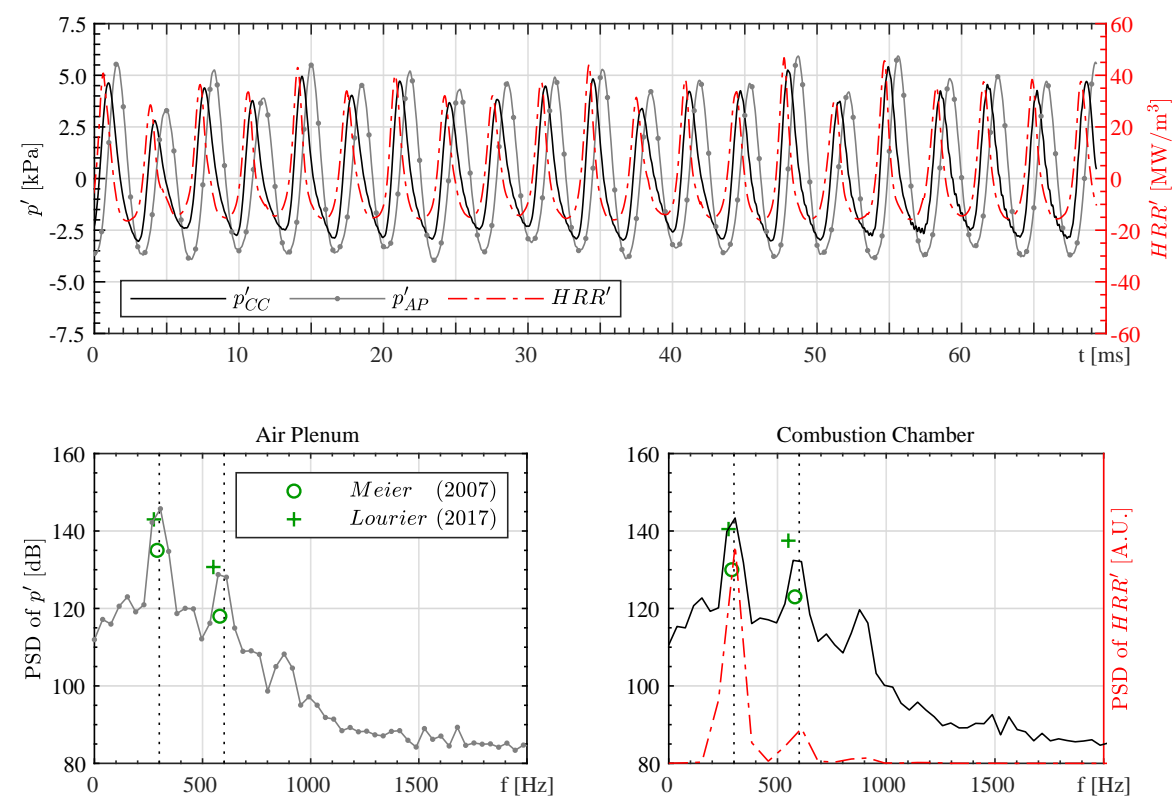

FIG. 3. Top: Time signals of the global heat release rate and local pressure fluctuations recorded at AP and CC. Bottom: Their respective power spectral densities. PSD peaks from the experimental pressure signals are plotted for measurements with vibrating quartz windows ${ }^{48}$ and rigid metal walls ${ }^{43}$

showing two pronounced peaks in the air plenum and combustion chamber pressures as well as in the global HRR. These peaks represent the dominant thermo-acoustic mode at $300 \mathrm{~Hz}$ and its first harmonic at $600 \mathrm{~Hz}$, closely matching the experimentally measured counterparts of approximately $290 \mathrm{~Hz}$ and $580 \mathrm{~Hz}^{48}$. The minor discrepancy of about $10 \mathrm{~Hz}$ may be related to a combination of small mismatches in the simulated test rig geometry (i.e. shorter inflow or exhaust pipe lengths), and the operating conditions, e.g. slightly higher air inflow temperatures increasing the local sound speed. Correct trends are also evident for the computed power densities. However, the peak amplitudes of $145 \mathrm{~dB}$ and $128 \mathrm{~dB}$ in the air plenum as well as $143 \mathrm{~dB}$ and $133 \mathrm{~dB}$ in the combustion chamber are each overestimated by about 10-13 dB when compared to the initial experiments by Meier et al. $^{48}$. A similar observation was reported in a recent simulation by Lourier et al. ${ }^{43}$ who 
associated the over-prediction with acoustic damping incurred at the loosely-fitted quartz glass windows used in the experiments. Additional measurements performed with tightlyfitted rigid metal walls subsequently showed an increase of the peak pressure amplitudes by more than $10 \mathrm{~dB}^{43}$. This significant increase ultimately yields excellent agreement for the two dominant modes predicted in the current work, which are respectively within $2 \mathrm{~dB}$ and $5 \mathrm{~dB}$ of these additional measurements. Note that the fuel composition may also have an impact on the combustor's limit-cycle behaviour. A recent study by Gong et al. ${ }^{86}$ has shown that even low levels of hydrogen enrichment can affect the frequency of the thermo-acoustic modes in the PRECCINSTA test case.

\section{B. Phase-averaged statistics}

The oscillating flow behaviour is quantified in Figs. 4, 5 and 6 via phase- and timeaveraged radial profiles of the velocity, temperature and species mass fractions at five different downstream locations. The statistics have been collected for a duration of $130 \mathrm{~ms}$ of physical time - equivalent to about 40 oscillation cycles - and are compared with measurement data from the initial experiments by Meier et al. ${ }^{48}$. As mentioned previously, these experiments were influenced by acoustic damping effects in the combustion chamber, which are neglected in the current simulation. The computed flame oscillation is therefore expected to be amplified as a result of the increased limit-cycle amplitude. Unfortunately, similar detailed measurement data from the latter campaign of Lourier et al. ${ }^{43}$ is not available since the utilised rigid metal walls provided no optical access for laser-based measurements. Nevertheless, both the axial and radial velocity profiles shown in Fig. 4 are in good agreement with the measurements and accurately reproduce the flow's oscillating behaviour. This includes reproducing the varying location and strength of the inner recirculation zone (IRZ), outer recirculation zone (ORZ) and incoming annular jet of fresh reactants, represented by two pronounced velocity maxima. Globally, these maxima display a minor inwards shift with downstream position, which, in combination with underestimated peak values in the radial velocity, indicate a smaller spreading angle. The jet's expansion into the combustion chamber is furthermore slightly over- and under-predicted between $P h_{8-2}$ and $P h_{4-6}$, respectively, exemplifying the amplified oscillation amplitude.

Figure 5 illustrates the corresponding flame oscillation, which is characterised by an al- 

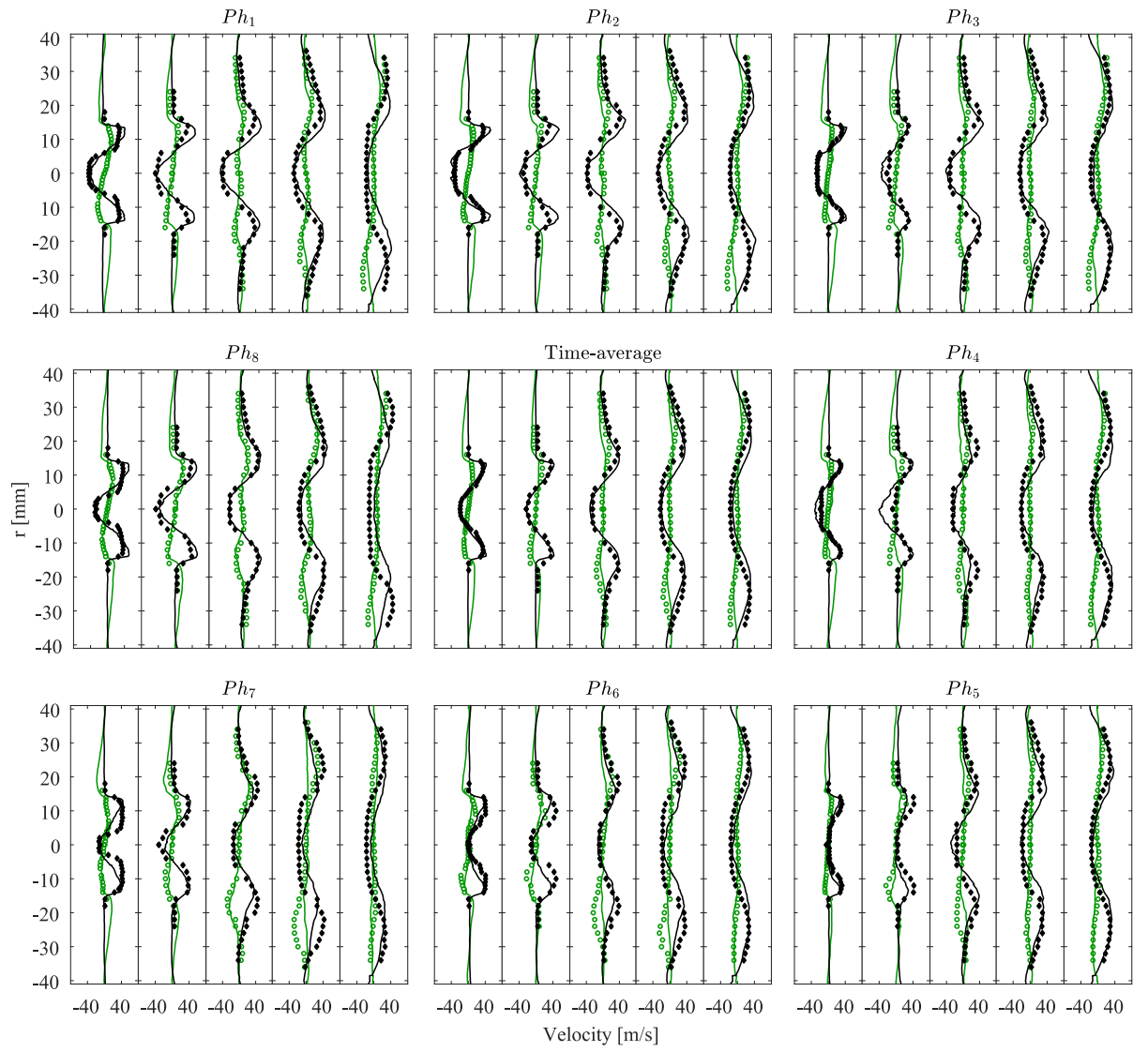

FIG. 4. Phase-averaged radial profiles of the axial (black) and radial (green) velocity at downstream positions $h=1.5,5,15,25,35 \mathrm{~mm}$ - lines: simulation; symbols: experiment. The corresponding time-averaged results are shown in the centre.

ternating down- $\left(P h_{8-2}\right)$ and upstream $\left(P h_{4-6}\right)$ movement of the flame front. The overall flame position and shape are qualitatively captured, while the temperature distribution in the ORZ is reproduced, suggesting the applied wall heat transfer treatment is realistic. Within the IRZ, the flame lift-off height is almost universally over-predicted, implying a longer duration of flame detachment ${ }^{23}$ from the burner nozzle cone compared to the measurements. In accordance with the obtained velocity field, an underestimation of the flame's 

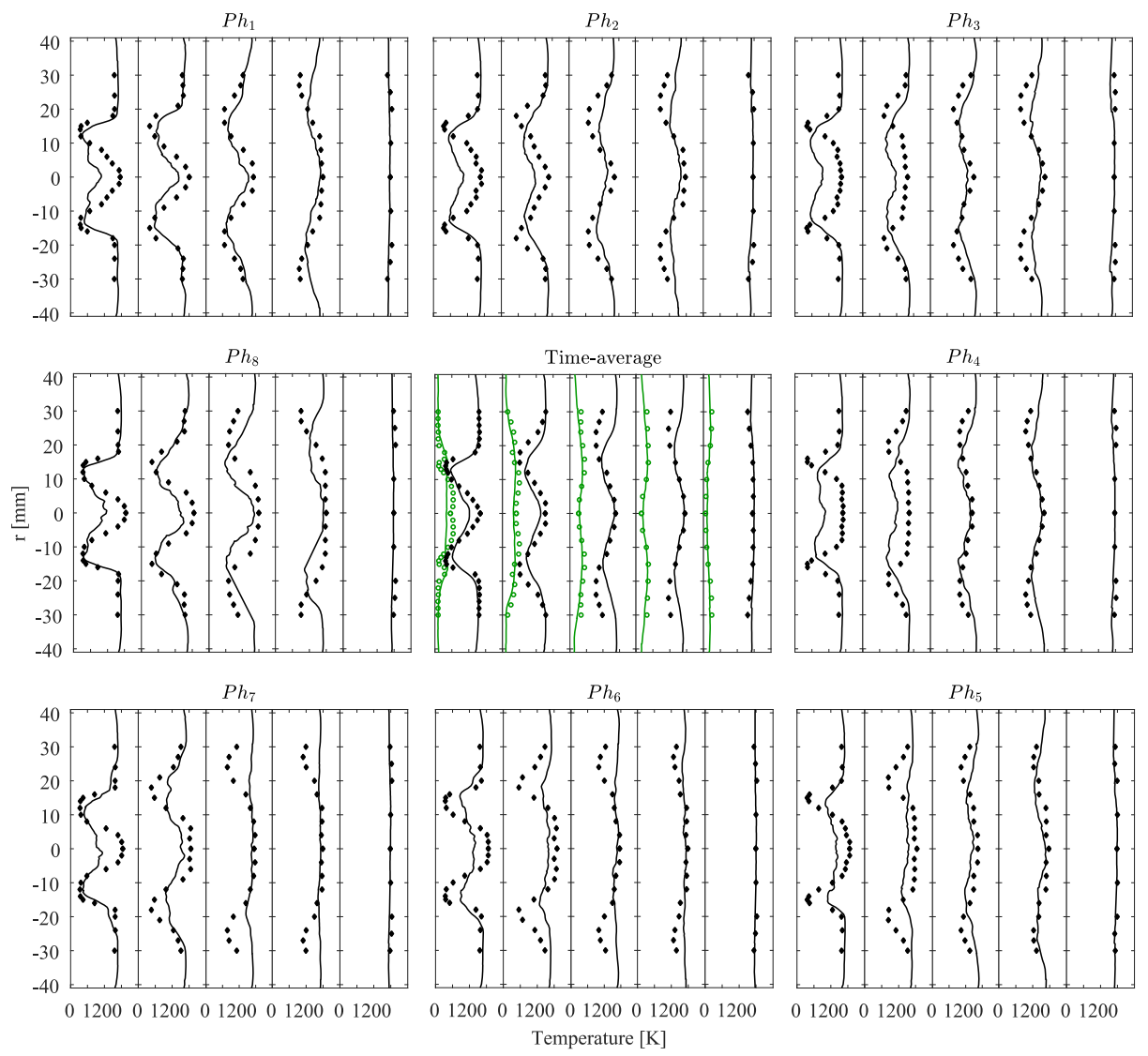

FIG. 5. Phase-averaged radial profiles of the mean (black) and RMS (green) temperature at downstream positions $h=6,15,25,35,60 \mathrm{~mm}$ - lines: simulation; symbols: experiment. The corresponding time-averaged results are shown in the centre.

global spreading angle can be determined from the time-averaged temperature profiles in the centre of Fig. 5. Moreover, global temperature fluctuations of up to $500 \mathrm{~K}$ - indicated by the RMS profiles - are expectedly high in both the simulation and experiments due to the oscillating nature of the flame. However, while the computed results show an almost constant level of fluctuation inside of the inner shear layer (ISL), $|r| \leqslant 16 \mathrm{~mm}$, experimental data reveals an additional dip of RMS values in between the ISL and outer shear layer (OSL), 10 

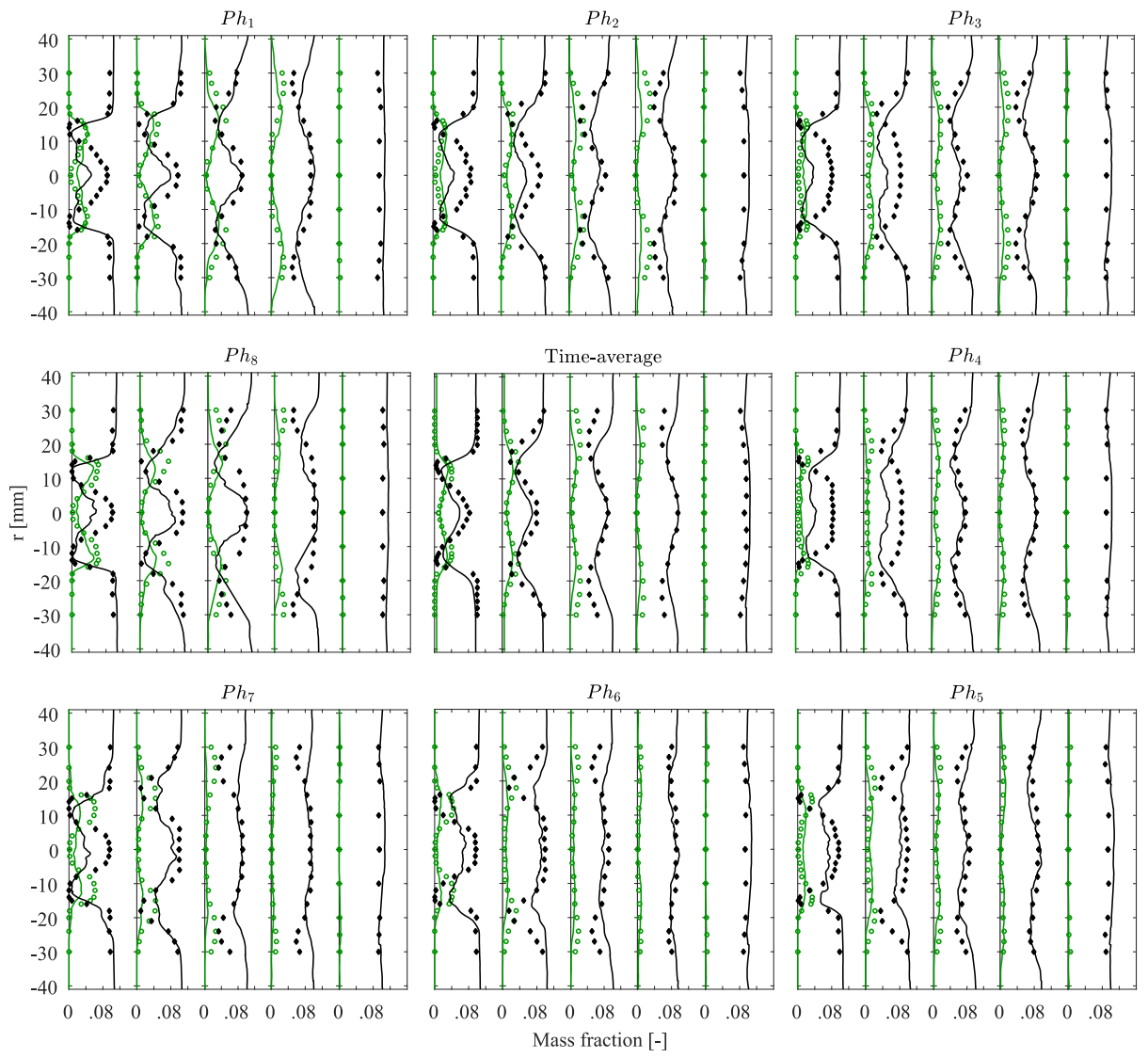

FIG. 6. Phase-averaged radial profiles of the $\mathrm{CO}_{2}$ (black) and $\mathrm{CH}_{4}$ (green) mass fraction at downstream positions $h=6,15,25,35,60 \mathrm{~mm}$ - lines: simulation; symbols: experiment. The corresponding time-averaged results are shown in the centre.

$\leqslant|r| \leqslant 16 \mathrm{~mm}$, not captured by the LES. The simulated radial location of the inner and outer flame front is therefore presumably less steady over the thermo-acoustic cycle. This conclusion is further supported by radial fluctuations of the ISL and OSL that are displayed in Fig. 4 and accompanied by a widening and narrowing of the IRZ. A distinct oscillation of the flame angle with respect to the axial direction - also known as flame flapping - can be inferred, which likely contributes to the under-predicted global spreading angle observed 
in the annular jet and flame. The increased flapping motion is potentially induced by swirl number fluctuations ${ }^{10,13}$ associated with stronger acoustic perturbations. These may again be attributed to a higher amplitude limit-cycle oscillation in the simulations. A detailed description of the swirl number fluctuation mechanism and the resulting flame angle oscillation is provided in Sections III E and III D, respectively.

Finally, radial profiles of the $\mathrm{CO}_{2}$ and $\mathrm{CH}_{4}$ mass fraction are shown in Fig. 6 to complement the velocity and temperature results. Although the overall trends between the different scalars are consistent, the deviations from the measurements are of larger magnitude compared to those of the velocity profiles. This is probably related to the large scalar gradients, for example in temperature, that occur across a thin flame front. Minor spatial differences of the flame location - manifested by small velocity deviations - will naturally result in more pronounced deviations in the composition and temperature profiles.

\section{Mass flow rate oscillation}

One full period of the global HRR, pressure and mass flow rate oscillations is presented in Fig. 7, including the different phase angles $\left(P h_{1-8}\right)$. The observed initial steep rise in heat release is expected to produce a pressure disturbance at the flame front that propagates up- and downstream within the domain. The local pressure, recorded by probes located in the combustion chamber and air plenum, increases accordingly with a specific time delay governed by the propagation speed of the acoustic wave. This time delay introduces a phase shift between both signals with a maximum phase difference of about $80^{\circ}$, similar to the experimental findings reported $\mathrm{in}^{48}$. The resulting pressure drop between the combustion chamber and air plenum fluctuates periodically inducing total mixture and $\mathrm{CH}_{4}$ mass flow rate oscillations inside the swirler and burner nozzle. These oscillations - evaluated in the burner nozzle exit plane $(h=0 \mathrm{~mm})$ - lead to a fluctuating supply of fresh reactants into the main flame zone, which in turn contributes to the unsteady heat release response. A minor phase lag between the two mass flow rates becomes evident and represents the temporal variation of the local mixture equivalence ratio. This phase lag is caused by an additional mechanism involving fuel accumulation inside the swirler unit combined with a convective time delay further discussed below.

The spatial and temporal evolution of the flow oscillation is shown in Fig. 8 for a full 

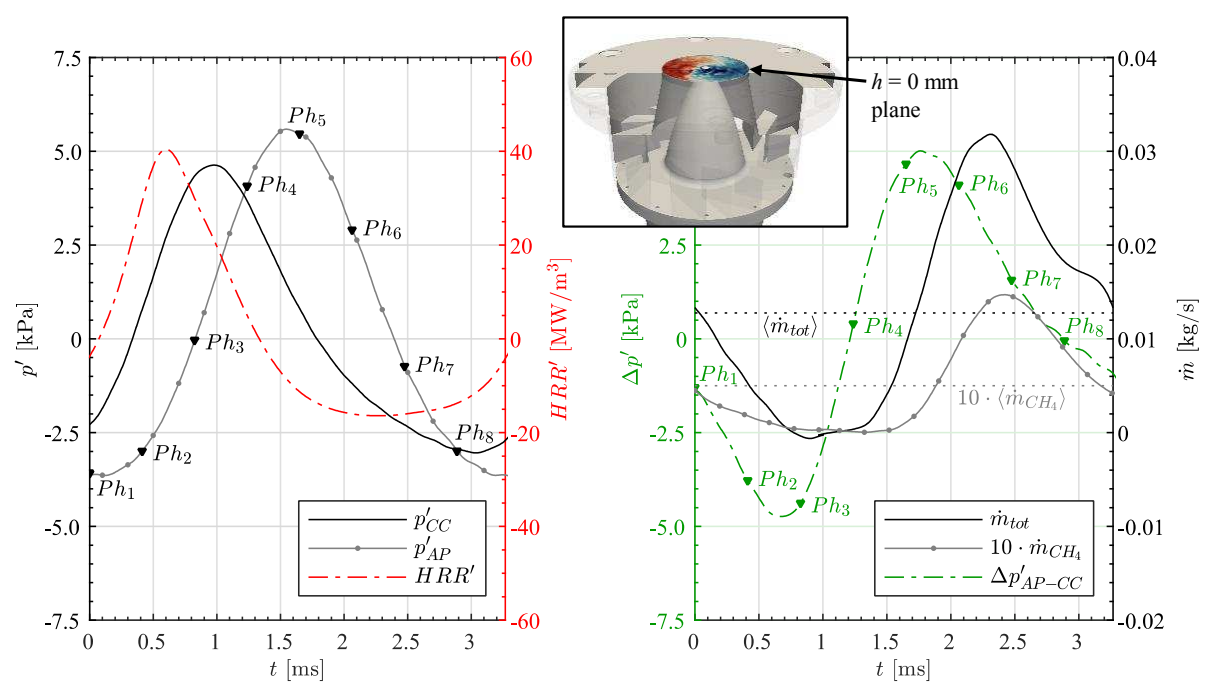

FIG. 7. Left: Temporal evolution of the global heat release rate and local pressure fluctuations at AP and CC for a full period of the oscillation cycle. Eight different phase angles are introduced: $P h_{1}=0^{\circ}, P h_{2}=45^{\circ}, P h_{3}=90^{\circ}, P h_{4}=135^{\circ}, P h_{5}=180^{\circ}, P h_{6}=225^{\circ}, P h_{7}=270^{\circ}, P h_{8}=315^{\circ}$. Right: Pressure drop between AP and $\mathrm{CC}$ as well as the resulting mass flow rate fluctuations of the fuel (scaled by a factor of 10) and total mixture in the burner nozzle exit plane $(h=0 \mathrm{~mm})$.

period of the thermo-acoustic cycle. Starting from $P h_{1}$, an increased mass flow rate of fresh reactants with elevated local equivalence ratio enters the combustion chamber - as characterised by the high velocity magnitudes and fuel concentration in this region. Large quantities of premixed fuel and oxidiser are thereby transported into the main flame zone enhancing combustion. The global HRR reaches its maximum value around $P h_{2}$, successively elevating the pressure inside the combustion chamber. Consequently, the pressure drop between the combustion chamber and air plenum becomes negative, decreasing the mass flow and hence supply of fresh reactants into the combustion zone. At $P h_{3}$, the bulk flow inside the swirler unit becomes almost stagnant, whereas the fuel injection rate remains essentially constant. This is a result of the high acoustic impedance of the fuel jets, making them only weakly susceptible to incident pressure disturbances. A significant amount of fuel accumulates within the swirler unit during $P h_{4-5}$, raising the equivalence ratio of the 


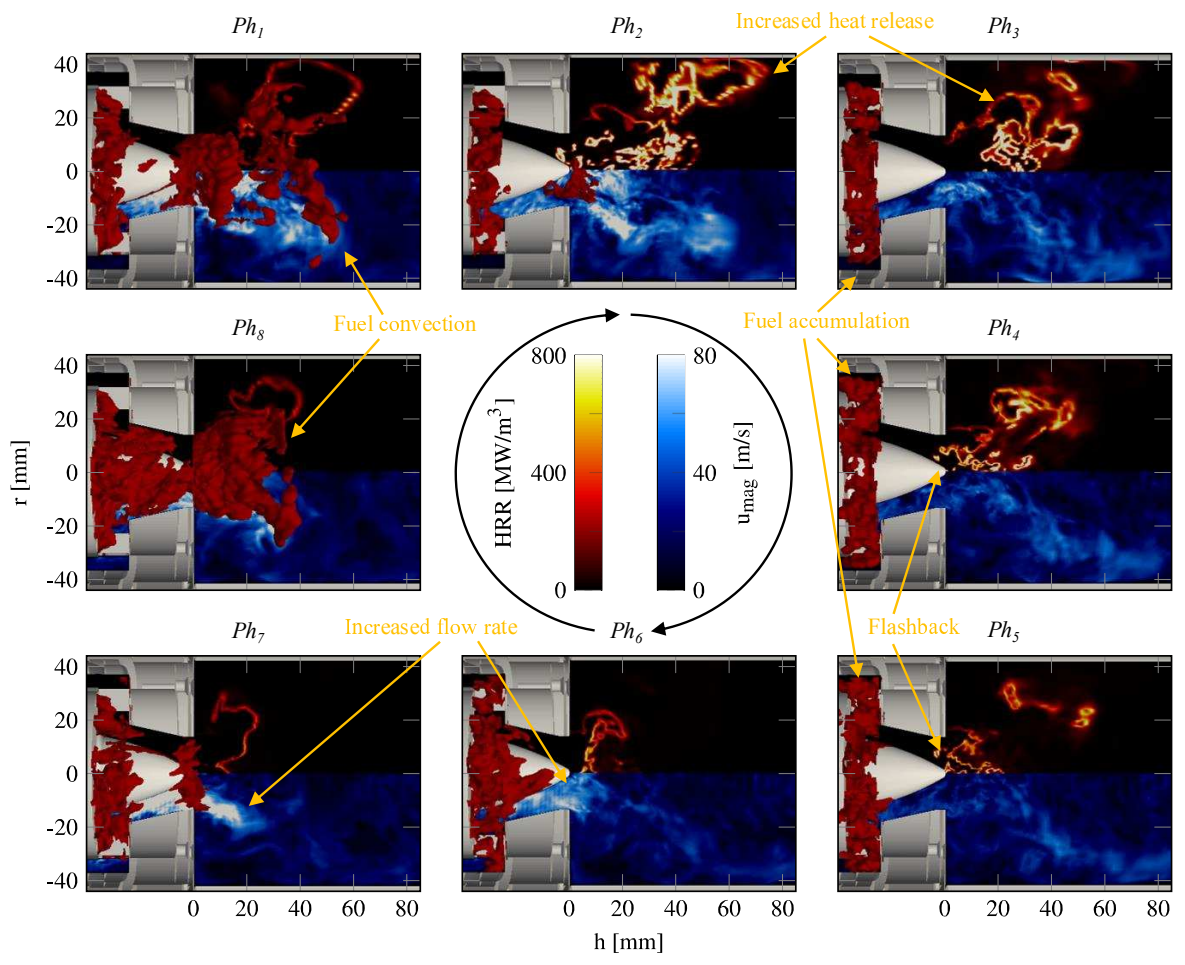

FIG. 8. Periodic evolution of the local heat release rate, velocity magnitude and reactant mixture composition. Red iso-surfaces of a constant $\mathrm{CH}_{4}$ mass fraction equal to 0.035 delineate the instantaneous fuel distribution.

local mixture. Scattered occurrences of flame flashback can be observed as the IRZ moves further upstream, extending the reverse flow far into the burner nozzle (cf. Fig. 14). Based on the lack of fresh reactants located within the combustion zone, the heat release rapidly decreases followed by a reduction of the local pressure. The pressure drop between the combustion chamber and air plenum is subsequently reversed and becomes positive. From $P h_{6}$, the mass flow rate within the swirler begins to increase again as the global HRR in the combustion chamber approaches a minimum. The accumulated high-equivalence-ratio mixture exits the burner nozzle with high momentum around $P h_{\gamma}$. Incidentally, the flame base begins to lift-off from the burner nozzle cone and outer rim while the flame front is 
convected downstream. Similar lift-off events have previously been reported in the literature, e.g. by Prasad et al. ${ }^{87}$ who investigated the propagation of lifted flames after a controlled mass flow pulse of fuel. The feedback loop is finally closed at $P h_{8}$ as the heat release begins to increase again. A superposition of mass flow rate and reactant mixture composition (i.e. local equivalence ratio) oscillations, can therefore be identified as the thermo-acoustic mechanism underlying the governing feedback loop. These oscillations are sustained by a periodically varying pressure drop between the combustion chamber and air plenum due to acoustic wave propagation.

\section{Unsteady flame dynamics}

The unsteady flame behaviour identified in the previous sections is further analysed using phase-averaged snapshots of the local OH concentration shown in Fig. 9. Some corresponding parameters, i.e. the axial jet extension into the combustion chamber, flame lift-off height and spreading angle at each phase are quantified in Fig. 10 by tracking the flame front based on a threshold value for the local HRR and $\mathrm{OH}$ concentration. The flame visibly lifts off (up to approximately $26 \mathrm{~mm}$ ) from the burner nozzle cone between $P h_{7-8}$ and reattaches about half of a period later at $P h_{3-4}$. Within each cycle, the overall flame topology transitions from an attached $\mathbf{V}$-shape $\left(P h_{4-6}\right)$ to a lifted $\mathbf{M}$-shape $\left(P h_{8-2}\right)$ and vice versa. This flame shape transition can be compared to the one observed experimentally by Oberleithner et al. ${ }^{53}$ and Stöhr et al. ${ }^{56}$ for a slightly different PRECCINSTA operating condition with reduced thermal power. In these experimental studies however, the transition was not periodic and instead involved a bi-stable flame undergoing bifurcation with significantly longer time scales (about once per second). Furthermore, a radial compression and expansion of the flame spreading angle can be identified and comes with a widening and narrowing of the flame base (and annular jet, cf. Fig. 4), which was also reported in recent experiments by Stöhr et al. ${ }^{9}$. The minimum and maximum flame angles of $62^{\circ}$ and $82^{\circ}$ are observed around $P h_{3}$ and $P h_{7}$, respectively. 

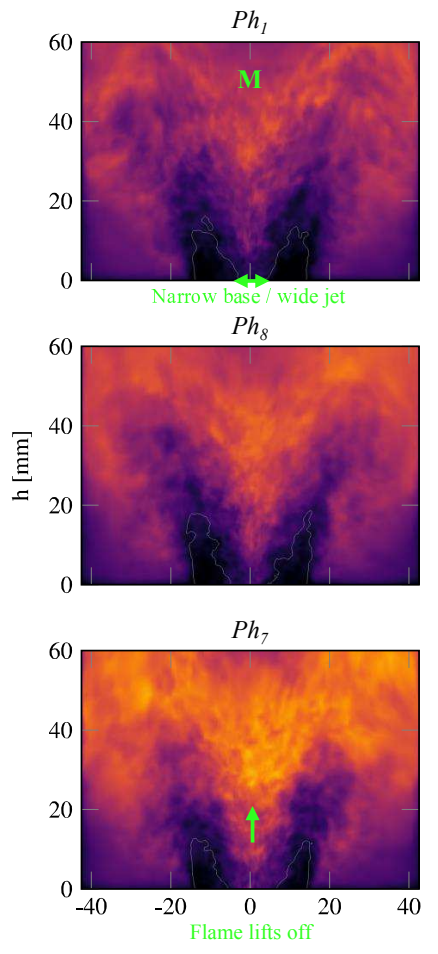

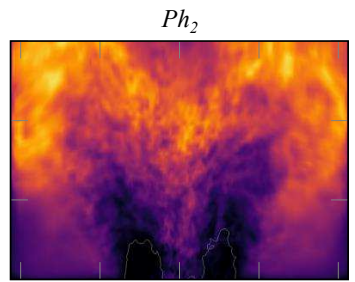

Time-average

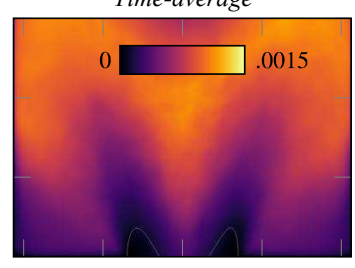

$P h_{6}$

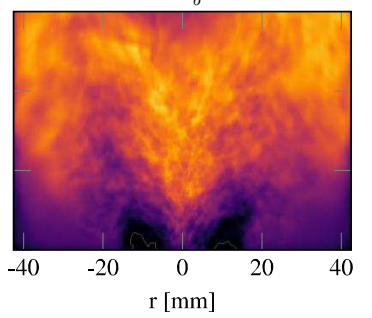

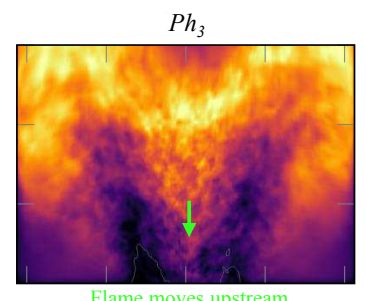

$\mathrm{Ph}_{4}$

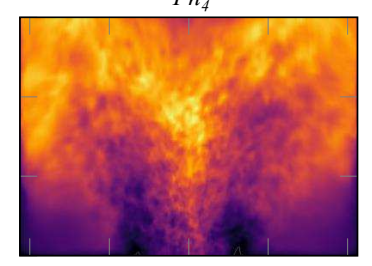

$P h_{5}$

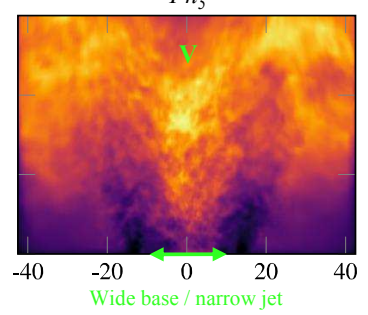

FIG. 9. Phase-averaged snapshots of the $\mathrm{OH}$ concentration with arrows qualitatively indicating the periodic evolution of the flame motion, spreading angle and overall topology (i.e. V-shape or M-shape). White iso-contours of a constant $\mathrm{CH}_{4}$ mass fraction equal to 0.025 indicate the extension of the incoming annular jet.

\section{E. Swirl number oscillation}

Previous works have shown that periodic fluctuations of the flame spreading angle may arise due to unsteady interactions between a swirler unit and incident acoustic perturbations. Under low-Mach number flow conditions, pressure waves that impinge on a swirler unit are mostly transmitted and subject to a mode conversion process giving rise to both axial and azimuthal velocity perturbations ${ }^{88}$. The former are propagated downstream (at the sound speed) in the form of acoustic waves. They may induce a shedding of toroidal vortices from 


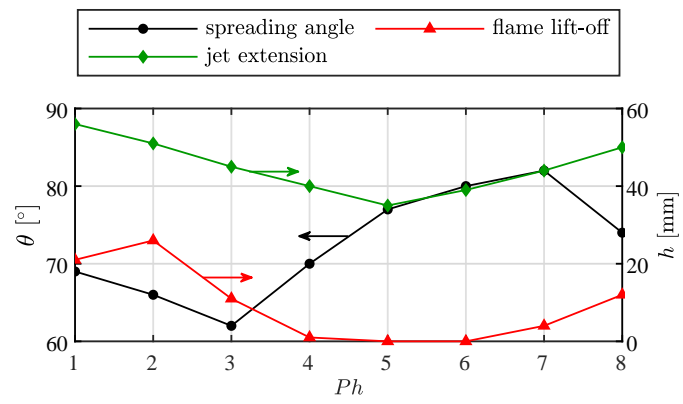

FIG. 10. The axial jet extension into the combustion chamber, flame lift-off height and spreading angle (evaluated at $h=20 \mathrm{~mm}$ ) for each phase.

the burner nozzle rim, which in turn can roll up the flame front. The latter are caused by vorticity waves generated at the trailing edge of the swirler blades ${ }^{89-92}$ and have an amplitude of the same order of magnitude as the incident acoustic disturbance ${ }^{88}$. These vorticity waves are convected downstream by the bulk flow and effectively perturb the swirl number, which controls the motion and strength of the IRZ ${ }^{13,93}$. The resulting angular oscillation of the flame root may then lead to fluctuations of the turbulent burning velocity, thereby promoting an unsteady heat release response ${ }^{94}$. The exact mechanisms underlying the mode conversion process are still not well understood and remain a major focus of ongoing research efforts.

The swirl number $S^{95}$ essentially compares the axial flux of azimuthal momentum with the axial flux of axial momentum and can be defined as:

$$
S=\frac{\int_{0}^{R} \rho u_{x} u_{\theta} r^{2} d r}{R \int_{0}^{R}\left(\rho u_{x}^{2}+p\right) r d r}
$$

where $r$ and $R$ represent the radius and burner nozzle equivalent radius, while $u_{x}$ and $u_{\theta}$ are the axial and azimuthal velocity components, respectively. The temporal evolution of the instantaneous swirl number is shown in Fig. 11 for a full period of the thermoacoustic cycle. It can also be deduced from the ratio of the axial and azimuthal momentum flow rates spatially integrated over the burner nozzle exit plane. The fluctuation of these momentum flow rates originates from the above-described mode conversion process induced by the periodically varying pressure drop between the air plenum and combustion chamber. The observed flame angle oscillation can therefore be related to a periodic fluctuation of the instantaneous swirl number in accordance with previous studies. Other hydrodynamic 


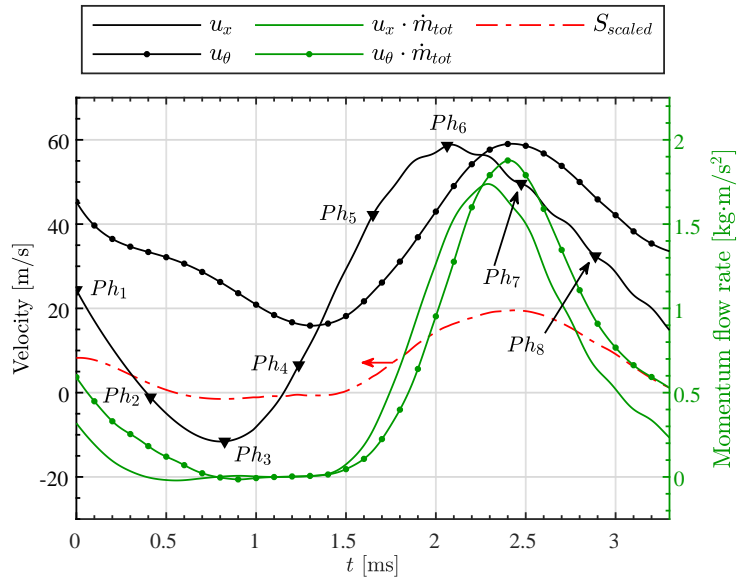

FIG. 11. Temporal evolution of the axial and azimuthal components of the velocity $\left(u_{x}, u_{\theta}\right)$ and momentum flow rates, spatially integrated over the burner nozzle exit plane $(h=0 \mathrm{~mm})$ for a full period of the oscillation cycle. The red dash-dotted line indicates the resulting oscillation of the instantaneous swirl number (scaled up for visualisation purposes).

phenomena such as a PVC or periodic vortex shedding have also been associated to the swirl-induced axial and azimuthal momentum in the past. These can have a significant impact on the local heat release, viz. by directly modulating the flame's 'surface area' or enhancing the mixing of fuel and oxidiser. A direct link, however, has yet to be established between variations of the instantaneous swirl number and the excitation or suppression of a PVC. The potential formation of coherent vortical structures - physically characterised by low pressure regions - in the current test case is investigated below. Despite introducing some ambiguity with respect to the size of these structures, it is common practice to visualise them via iso-surfaces of the pressure ${ }^{66}$.

\section{F. Coherent hydrodynamic structures}

Figure 12 shows the local excitation and suppression of large-scale vortical structures in the combustion chamber for each phase. At $P h_{3}$, the axial and azimuthal momentum flow rates entering the combustion chamber reach a minimum (cf. Fig. 11) and no distinct hydrodynamic structure can be detected in the region of interest. A central vortex core (CVC) 

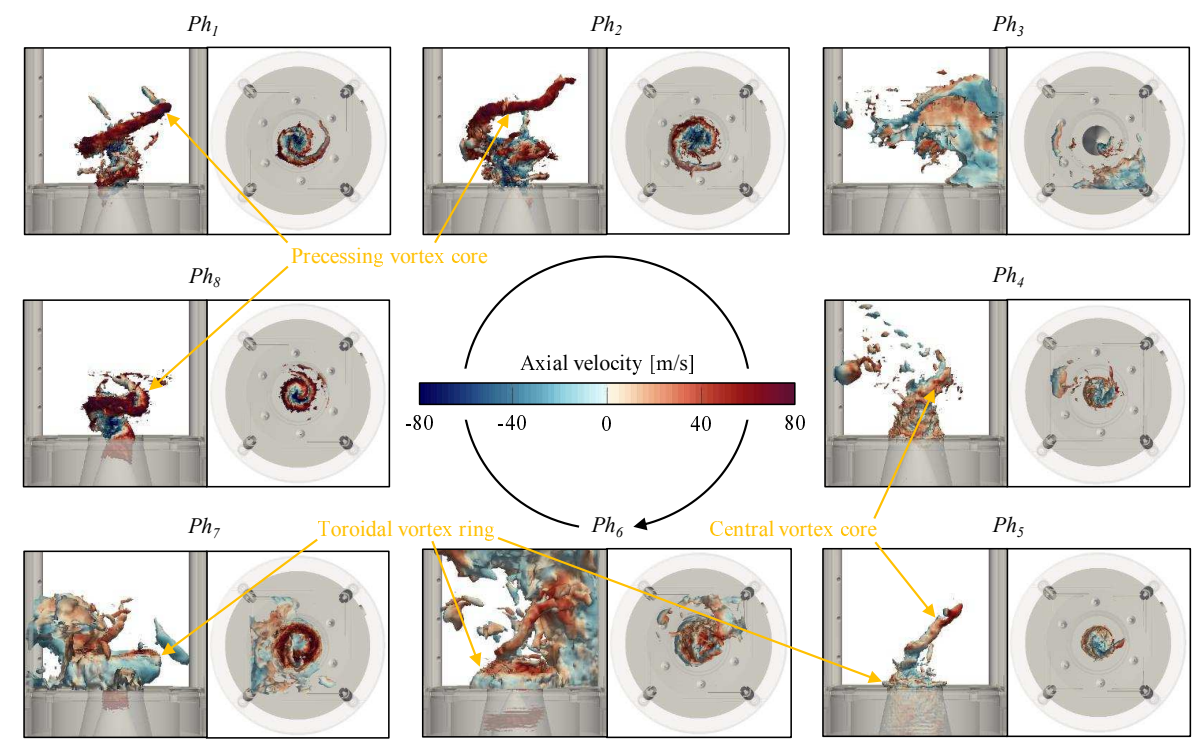

FIG. 12. Periodic formation and suppression of large-scale coherent vortex structures inside the combustion chamber (top and side view) visualised by local low pressure iso-surfaces and coloured by axial velocity.

is observed throughout the rest of the oscillation cycle $\left(P h_{4-2}\right)$ sustained by the azimuthal momentum induced in the swirler unit. The steadily increasing axial momentum from $P h_{5}$ leads to the development of a vortex ring adjacent to the outer rim of the burner nozzle. This vortex ring continues to gain in size until it is eventually shed from the rim after $P h_{6}$ and convected downstream in the shape of a toroid. Between $P h_{8-2}$, a helical flow motion is excited by the rapidly increasing azimuthal velocity generated from the vorticity wave exiting the burner nozzle. As seen in Fig. 11, the ratio between azimuthal and axial momentum flow rate, i.e. the instantaneous swirl number inside the burner nozzle, becomes largest leading up to this point. The observed helical structure is usually referred to as PVC and its existence has been confirmed for a number of swirl-stabilised combustor configurations, see e.g. Syred ${ }^{19}$ (note that very few previous numerical works have successfully detected a PVC under reacting flow conditions: $:^{20,23,44,96}$ ). As the instantaneous swirl number decreases again, the PVC performs an almost $180^{\circ}$ rotation while increasing its axial and radial extension 
into the combustion chamber until it finally breaks down and vanishes before $P h_{3}$. Hence, a direct link appears to be prevalent between the formation of the PVC and the strength of the instantaneous swirl number. To the best of the authors' knowledge, up to now this relationship had not been confirmed in the open literature, and brings forth important implications for the design of lean swirl-stabilised combustion systems. The above-described loop of hydrodynamic interactions is repeated at each oscillation cycle showing its strong coupling with the predominant thermo-acoustic mode.

To further demonstrate the periodic nature and excitation of the observed coherent vortex structures, a spectral proper orthogonal decomposition (SPOD) method $^{97}$ was applied to the pressure field in both a vertical and axial plane inside the combustion chamber. The analysis provides the SPOD energy spectrum at discrete frequencies, namely the limit-cycle oscillation frequency, $f=300 \mathrm{~Hz}$ and its higher harmonics, as well as the respective spatial mode shapes. The results indicate that at $f=300 \mathrm{~Hz}$, most of the energy is contained within the first SPOD mode (not shown here) with a relative modal energy, $\lambda$ of about $99 \%$. This first mode represents the afore-mentioned longitudinal thermo-acoustic instability, whereas all subsequent higher modes can be associated to the different hydrodynamic phenomena. For example, the previously identified PVC can be found in SPOD mode 3, which is shown in Fig. 13 for the discrete frequency range of $f=300,600,900,1200,1500 \mathrm{~Hz}$. The characteristic 'zig-zag' shape observed in the vertical plane (analogous to an axisymmetric shape when considering the transversal velocity component instead of the pressure) is typical for an asymmetric hydrodynamic instability mode. Snapshots of the axial plane further show a 'yin and yang'-type pattern at $f=600$ and $900 \mathrm{~Hz}$, similar to the one reported in Moeck et al. ${ }^{21}$ for a global helical mode. Overall, the obtained spatial mode shapes coincide well with previous observations of PVC structures in swirl-stabilised combustors. Note that while the relative modal energies of the hydrodynamic modes increase with higher frequencies, the total SPOD energy content of these modes is significantly lower compared to that of the predominant longitudinal thermo-acoustic mode. The observed coherent vortex structures are therefore also expected to have a relatively minor effect on the unsteady global heat release rate. Nevertheless, the specific roles and importance (i.e. local effects) of the PVC and toroidal vortex shedding mechanism within the combined oscillation cycle are further discussed in the following sections. 

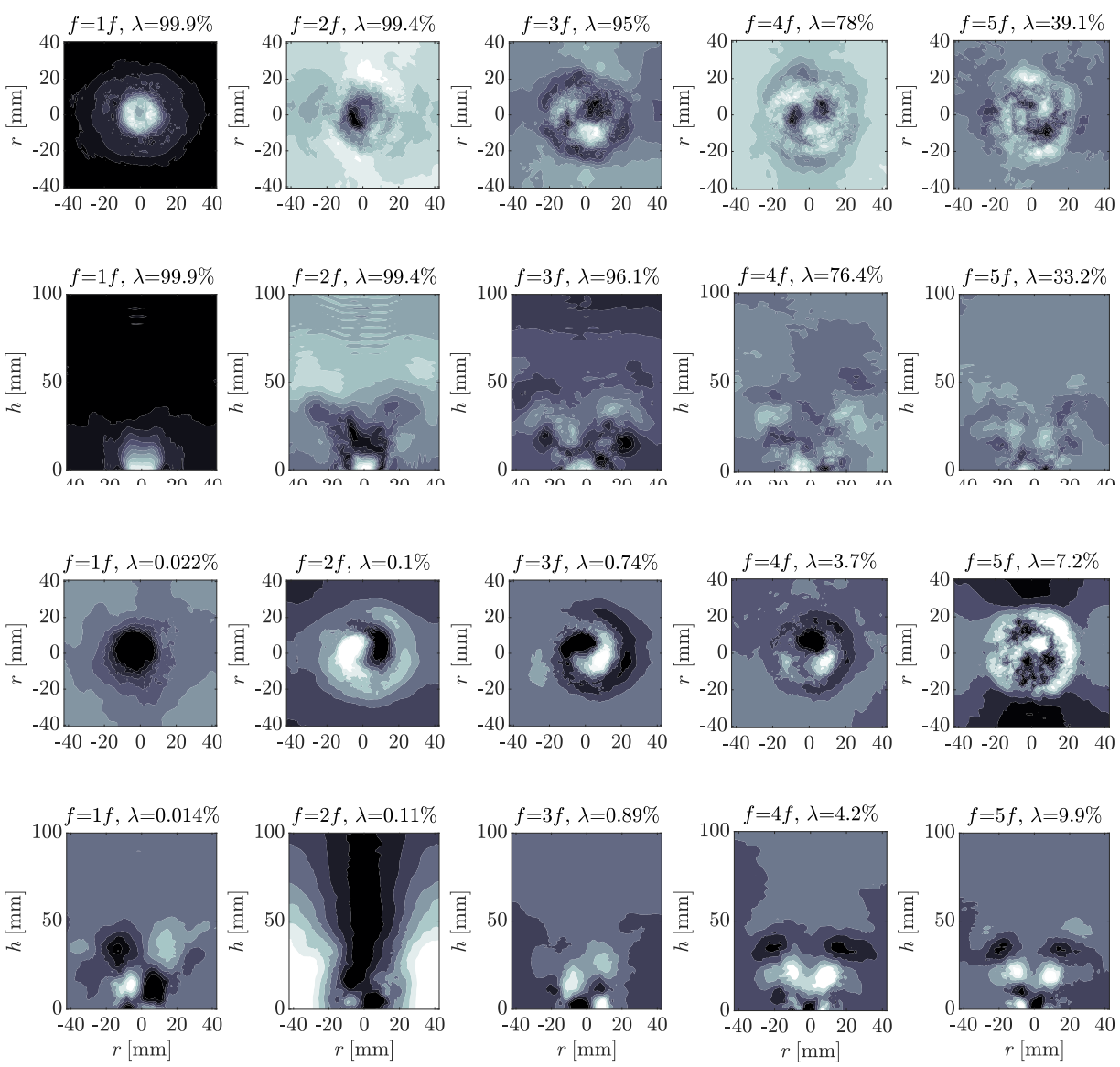

FIG. 13. Spatial mode shapes corresponding to the first (top two rows) and third (bottom two rows) SPOD mode of the pressure field evaluated in an axial plane at $h=20 \mathrm{~mm}$ and a vertical plane through the central burner axis. Left to right: modal frequencies, i.e. harmonics of the limit-cycle oscillation frequency, $f=300 \mathrm{~Hz}$, along with the respective relative modal energies, $\lambda$ expressed as a percentage of the discrete SPOD energy spectrum.

\section{G. Precessing vortex core}

Figure 14 shows the periodic evolution of the PVC and its interaction with the flame front. As expected, the PVC is situated along the zero velocity line separating the annular 


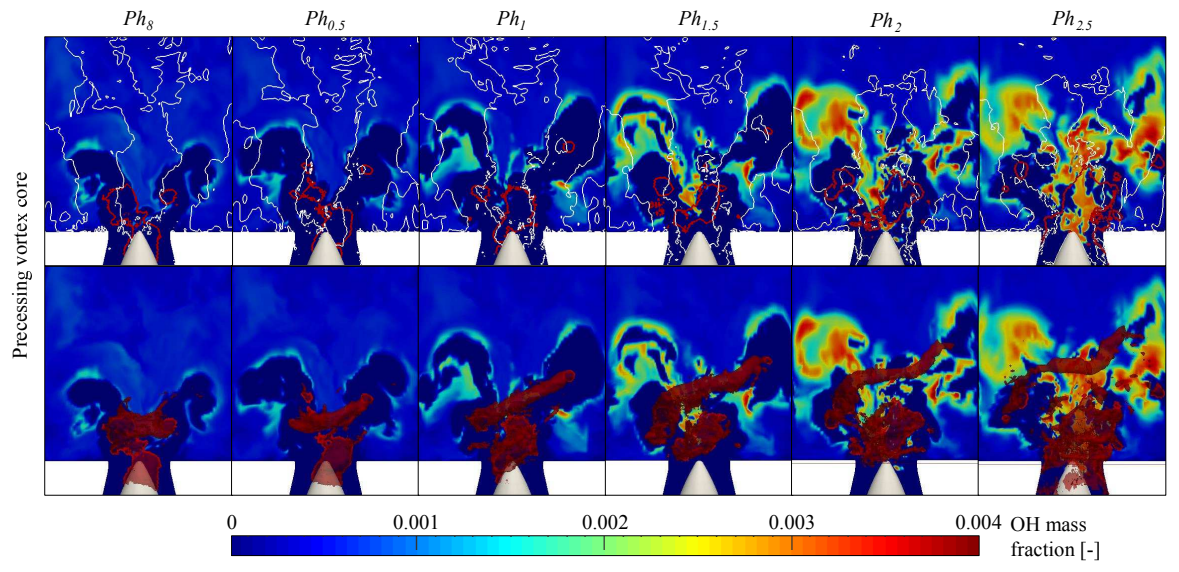

FIG. 14. Periodic evolution $\left(P h_{8-2.5}\right)$ of the PVC and its interaction with the flame front visualised by $\mathrm{OH}$ concentration. Bottom half: three-dimensional vortex visualisation; top half: vertical slice through the vortex. Red iso-contours delineate the vortical structures via low isobaric values whereas white iso-contours indicate the zero velocity line.

jet of reactants and the IRZ ${ }^{19}$, while causing the characteristic asymmetric deformation / displacement of the latter. This deformation is a result of the swirl-induced asymmetric vortex breakdown underlying the vortex core precession around the central burner axis ${ }^{98}$. The flame generally stabilises in between the ISL and OSL, except for instances when it is locally displaced by the PVC (visible at $P h_{2}$ ), consequently generating asymmetric combustion. It becomes clear that the vortex core precession also coincides with flame detachment and lift-off from the burner nozzle cone as previously observed, e.g. in Refs. ${ }^{23,99,100}$. The PVC appears to play a role in this detachment mechanism by increasing the fluid strain rate leading to local flame extinction ${ }^{57}$. The resulting region of unburnt, low viscosity gases with a fairly uniform density distribution serves as a favourable environment for PVC formation. Schönborn et al. ${ }^{24}$ have recently suggested that flame eccentricity caused by vortex core precession can be detrimental to upstream flame propagation and thus effective in inhibiting flame flashback. However, as a result of the increasing reverse flow from $P h_{2}$, the inner flame front eventually surges back upstream towards the burner nozzle cone. Enhanced fuel-air mixing induced by the PVC possibly accelerates this process by promoting upstream flame 


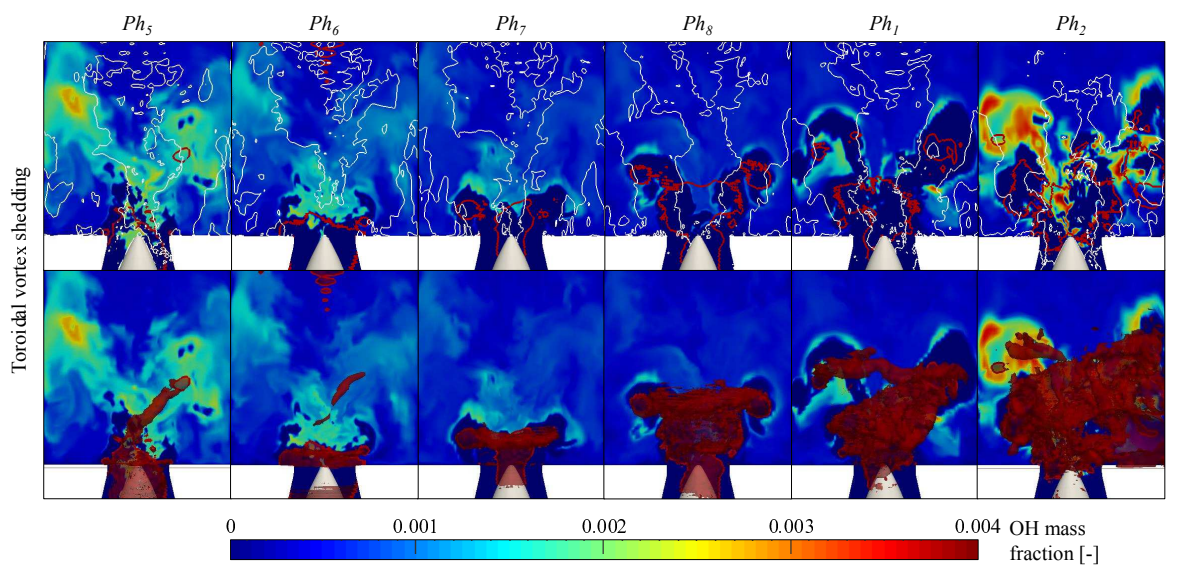

FIG. 15. Periodic evolution $\left(P h_{5-2}\right)$ of the toroidal vortex and its interaction with the flame front visualised by $\mathrm{OH}$ concentration. Bottom half: three-dimensional vortex visualisation; top half: vertical slice through the vortex. Red iso-contours delineate the vortical structures via low isobaric values whereas white iso-contours indicate the zero velocity line.

propagation in the region ${ }^{101}$. The PVC base is incidentally 'blown off' the burner nozzle cone and pushed outward allowing the inner flame front to reattach. This in turn generates strong radial density/temperature gradients, which have proven to be critical in suppressing vortex core precession ${ }^{53,102}$. The entire PVC structure ultimately vanishes after $P h_{2.5}$ as the flame begins to flash back into the burner nozzle.

\section{H. Toroidal vortex shedding}

A description of the periodic vortex shedding mechanism is carried out based on Fig. 15 . As mentioned above, from $P h_{5}$ a low pressure ring vortex develops on the outer rim of the burner nozzle due to flow separation stemming from an increased mass flow rate entering the combustion chamber. This ring vortex consistently grows in size and appears to assist local quenching of the flame base around the OSL, which eventually leads to complete flame detachment from the outer rim. The ring structure itself is shed between $P h_{6-7}$, forming a toroidal vortex that is convected downstream along the OSL at the bulk flow velocity. It continues to grow under the influence of vortex stretching, exothermicity and volumetric 
expansion ${ }^{103}$ while interacting with the flame's reaction layers. At $P h_{1}$, the toroidal vortex collides with the helical PVC structure resulting in its partial destruction before it eventually breaks down and dissipates after $P h_{2}$.

Prior to the present work, Steinberg et al. ${ }^{50}$ experimentally detected a PVC and symmetric vortex shedding in a parametric study of the PRECCINSTA combustor. Both phenomena however, did not co-exist at the same combustor operating condition. The symmetric shedding of toroidal vortices was obtained at lower thermal powers, whereas higher thermal powers led to the formation of the PVC. In the current work, periods of reduced heat release during the oscillation cycle $\left(P h_{5-8}\right.$; cf. Fig. 7$)$ can be associated with lower thermal powers promoting vortex shedding. As the global HRR increases $\left(P h_{8-2}\right)$ - corresponding to an operating condition with higher thermal power - the PVC is excited instead. A higher limitcycle amplitude compared to the experiments conducted with the loosely-fitted quartz glass combustion chamber walls further contributes to this effect by enhancing the fluctuation intensity. The simultaneous existence of both phenomena at the same operating condition can thereby be explained.

\section{Flame-vortex interaction}

A detailed representation of the typical instantaneous interaction between the flame, flow and large-scale vortices is provided in Fig. 16. The PVC and toroidal vortex both visibly roll up the flame front, thereby directly modulating its overall 'surface area' and generating unsteady heat release in an asymmetric or axisymmetric manner, respectively. However, Balachandran et al. ${ }^{104}$ have shown that vortices induced by inlet velocity fluctuations may not only generate 'surface area' when the flame front wraps around them, but can also lead to its destruction through flame annihilation events. A distinct hole in the flame sheet is created by the toroidal vortex enclosing unburnt reactants within its core. These trapped reactants are convected downstream and consumed after a time delay governed by the vortex break-up time. Steinberg et al. ${ }^{105}$ investigated the re-ignition of flame holes due to edge-flame propagation around the perimeter of such vortical structures, indicating well-mixed pockets of fuel and oxidiser. A rapid growth in heat release once these pockets of trapped reactants are consumed by flame fronts converging towards their centres was reported by Ghoniem et al. ${ }^{106}$. Flame-wall interaction in the present case appears to form additional reactant 


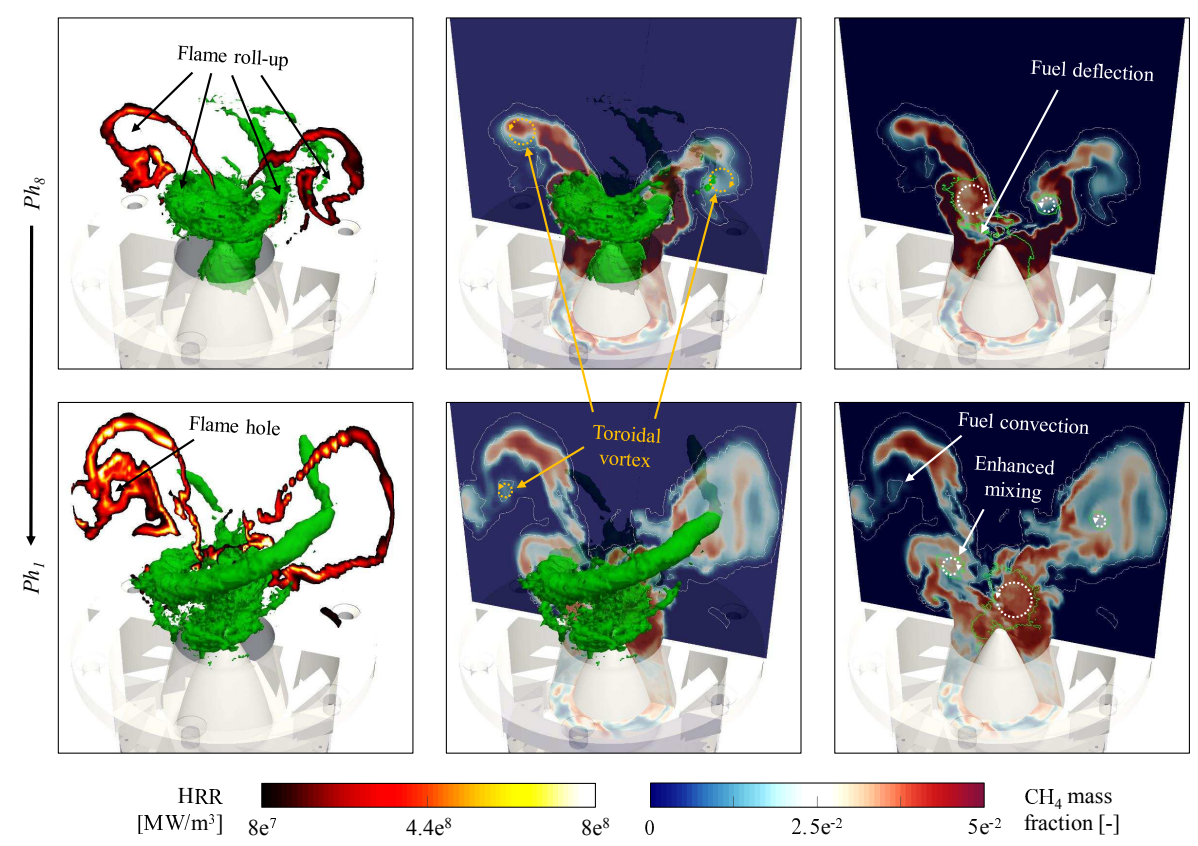

FIG. 16. Typical instantaneous interaction of the PVC and toroidal vortex with the flame front and the incoming annular jet at $P h_{8}$ (top) and $P h_{1}$ (bottom). Green iso-contours delineate the vortical structures via low isobaric values. White iso-contours indicate a constant local heat release rate of $80 \mathrm{MW} / \mathrm{m}^{3}$ corresponding to about $5 \%$ of its maximum value during this period of the oscillation cycle.

pockets that are entrained between the flame front and combustion chamber side walls and therefore also subject to delayed consumption. The PVC may further influence combustion by enhancing the local mixing of fuel and oxidiser ${ }^{25}$ and, due to its positioning along the ISL, supplying heat and radicals from the recirculating burnt gases to the incoming stream of unburnt reactants ${ }^{107}$. The incoming stream is moreover radially deflected by the $\mathrm{PVC}^{108}$ in proximity of the burner nozzle resulting in asymmetric heat release downstream. This deflection can assist upstream flame propagation by reducing the flame-normal approach speed of the incoming annular jet ${ }^{109}$.

Prior studies have also suggested that helical PVC structures conventionally do not con- 
tribute to fluctuations of the global HRR, implying that any local influence cannot be detected in the spatially integrated signal. This is related to their non-axisymmetric azimuthal mode shape - i.e. $m \neq 0$, when expressed as a flow disturbance in normal form using cylindrical coordinates:

$$
\Upsilon^{\prime}(x, r, \theta, t)=\sum_{m=0}^{\infty} \check{\Upsilon}_{m}(r) \exp [i(k x+m \theta-\omega t)]
$$

where $\Upsilon^{\prime}$ represents any disturbance quantity, $\check{\Upsilon}_{m}$ is the azimuthal disturbance amplitude, $k$ and $m$ are the axial and azimuthal wave number, respectively, and $\omega$ is the frequency of the disturbance, see e.g. ${ }^{110}$. In this formulation, the PVC corresponds to a helical mode of $m=1$, and thus exerts a non-axisymmetric modulation of the flame 'surface area'. The associated fluctuations in local heat release must therefore be strongly asymmetric and have previously been shown to cancel out in space over the oscillation period ${ }^{21,111}$. However, such a spatial cancellation can only occur for a consistently active disturbance that constantly rotates about the central burner axis. In the current case, the PVC is periodically excited and suppressed, resulting in a phase-specific influence on the global HRR. The toroidal vortex, on the other hand, represents an axisymmetric mode $(m=0)$. It therefore modulates the local heat release in an axisymmetric manner through flame quenching and roll-up, causing fluctuations of the global HRR at the vortex shedding frequency. Hence, the successive formation of toroidal vortices and helical PVCs induces flame 'surface' disturbances with an $m=0$ to $m=1$ mode shape transition. Due to their favourable timing within the oscillation cycle, both hydrodynamic phenomena amplify the global instability by increasing heat release in phase with the dominant thermo-acoustic mechanism, thereby feeding acoustic energy into the system.

\section{J. Combined oscillation cycle}

In a simple analysis investigating vortex shedding without swirl, Venkataraman et al. ${ }^{17}$ have revealed that heat release oscillations are associated with both flame 'area' and equivalence ratio fluctuations. This finding has since also been confirmed for configurations involving swirling flames, see e.g. a recent LES study by Tachibana et al. ${ }^{42}$. In order to quantify these two parameters, the instantaneous flame 'sheet' is extracted in the current work by introducing a local HRR threshold of $1.5 \times 10^{8} \mathrm{~W} / \mathrm{m}^{3}$. This value corresponds to about $5 \%$ 

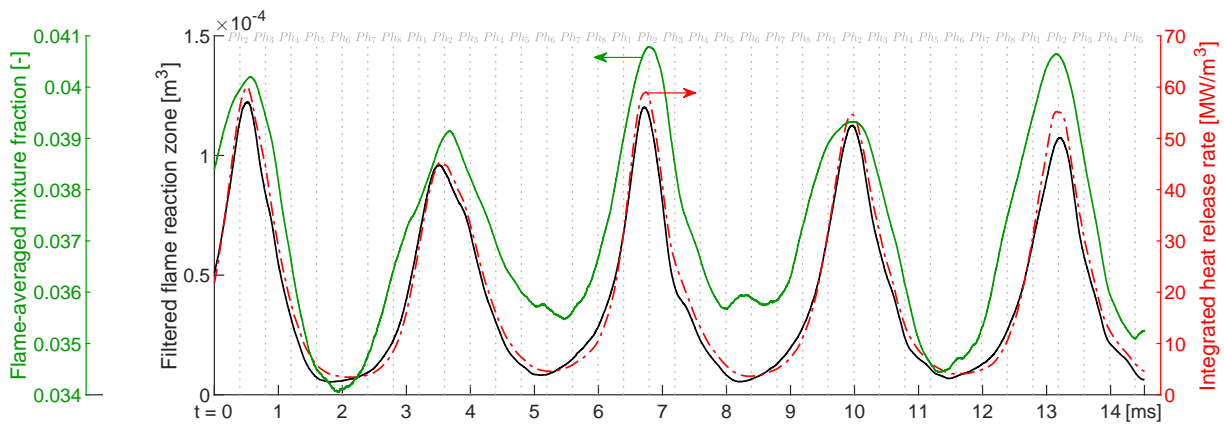

FIG. 17. Time signals of the filtered flame reaction zone (spatially integrated using a threshold of $150 \mathrm{MW} / \mathrm{m}^{3}$ on the local heat release rate), flame-averaged mixture fraction and global heat release rate.

of the maximum generated at any point in the chamber over the entire oscillation period. An approximation to the flame's 'surface area' is then obtained from the three-dimensional volume in which this threshold is exceeded - the filtered flame reaction zone volume. The mixture fraction can then be spatially integrated over this reaction zone volume leading to a measure of the flame-averaged mixture fraction (or equivalence ratio).

Figure 17 shows both the flame 'surface area' and flame-averaged mixture fraction quantified over multiple oscillation cycles alongside the global HRR (the corresponding periodic evolution of the instantaneous flame topology can be seen in Fig. 2). All three signals visibly fluctuate in phase, indicating that the heat release oscillation is indeed controlled by variations in the reaction rate per unit flame 'area' as well as the flame 'surface area' itself. The former are modulated by the equivalence ratio oscillation mechanism resulting from periodic fuel accumulation in the swirler unit. The latter can be related to the periodic generation and destruction of flame 'surface' due to large-scale elongation and corrugation of the flame front, ignition of unburnt reactants, and local flame extinction ${ }^{51}$. In the present work, these effects arise from hydrodynamic flame-flow-vortex interactions and the total mixture mass flow rate oscillation. Small-scale wrinkling of the reaction layers introduced by small-scale turbulence is an additional factor weighing into potential perturbations of the flame's 'surface area', though this may not be directly correlated with the prevailing limitcycle oscillation. Random turbulent events can however act as an initial trigger mechanism 


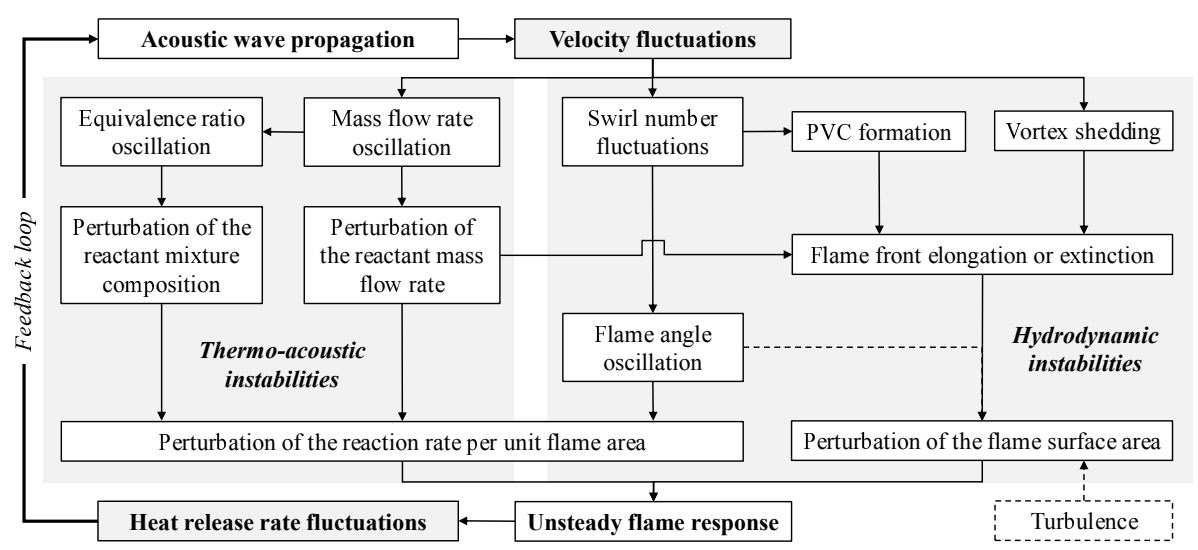

FIG. 18. Schematic overview of the different thermo-acoustic and hydrodynamic instability mechanisms and their role in sustaining the governing feedback loop underlying the limit-cycle oscillation.

for self-excited combustion instabilities.

The overall sequence of periodic amplification and attenuation can be summarised as follows: $P h_{4}$ - the global HRR, flame 'surface area' and flame-averaged mixture fraction all decrease rapidly; $P h_{5}$ - the flame approaches its minimum spreading angle, consequently reducing the reaction rate per unit flame 'area'; the developing vortex ring leads to additional flame 'surface' destruction by quenching the outer flame base; $P h_{6}$ - the growing mass flow rate of incoming reactants enhances combustion and elongates the flame front; the combination of low flame-averaged mixture fraction and increased stretch rates from higher velocities provokes partial extinction of the flame base ${ }^{112} ; P h_{7}$ - the subsequent rise in local mixture equivalence ratio reaches the flame front elevating its reaction rate; the shed toroidal vortex gains in size and strength as it is convected downstream generating flame 'surface' by rolling up the flame front; $P h_{8}$ - the helical PVC structure is formed inducing additional non-axisymmetric flame roll-up; $P h_{1}$ - the flame angle becomes maximum, further increasing the reaction rate per unit flame 'area'; $P h_{2}$ - the global HRR reaches its peak while the local mixture mass flow rate and equivalence ratio begin to decrease again; $P h_{3}$ - the toroidal vortex and PVC are both completely suppressed and the entire sequence of events repeats itself from $P h_{4}$. The full governing feedback loop including the various instability mechanisms identified in the above sections is summarised schematically in Fig. 18. 


\section{CONCLUSIONS}

Simulations of a self-excited flame oscillation cycle involving several thermo-acoustic and hydrodynamic instability mechanisms were performed using LES. The target test case, the PRECCINSTA combustor, consisted of a partially premixed, swirl-stabilised flame and has been previously shown to exhibit combustion-driven instabilities under the investigated operating conditions. The in-house BOFFIN-LESc code was applied in a fully compressible formulation. It employs a modelled transported $p d f$ equation, which is solved via the stochastic fields method to represent turbulence-chemistry interactions. The approach provides a burning regime independent description of turbulent flames and utilises a reduced 15-step / 19 species chemical mechanism.

First, the LES method's capability to reproduce complex, non-linear thermo-acoustic and hydrodynamic instabilities was demonstrated. The method successfully captured a fully selfexcited limit-cycle oscillation without requiring any external forcing. In line with previous experimental observations, the local heat release rate and combustion chamber pressure were found to fluctuate in phase, thereby globally satisfying the Rayleigh criterion. The frequency and amplitude of the predominant thermo-acoustic mode $(300 \mathrm{~Hz})$, as well as its first harmonic, were in excellent agreement with the available measured data. Phase-averaged radial profiles of the velocity, temperature and species mass fractions were furthermore acquired to quantify the dynamic behaviour of the flow field and showed reasonable agreement with the corresponding experimental results.

The observed oscillation cycle featured regular flame flashback and lift-off events as well as a periodic transition between an attached $\mathbf{V}$-shape and a lifted $\mathbf{M}$-shape flame. A superposition of mass flow rate and equivalence ratio oscillations - governed by a periodically varying pressure drop between the air plenum and combustion chamber - was identified as the main contributor to the underlying feedback loop. Moreover, an oscillation of the instantaneous swirl number was quantified and its role in the formation of various hydrodynamic flow phenomena, i.e. a flame angle oscillation, precessing vortex core and toroidal vortex shedding was studied. To the best of the authors' knowledge, this is the first time such a correlation between the instantaneous swirl number and the excitation and suppression of a PVC has been confirmed. Different coherent vortex structures arose and evolved

periodically, thus establishing a direct link to the predominant longitudinal thermo-acoustic 
mode.

Finally, the results of the simulations are consistent with existing knowledge in the field of combustion-driven instabilities. The visualisation, description and quantification of the physical mechanisms reported in this work provides further grounds for the confirmation of previous findings in the open literature. In particular, the identified PVC and toroidal vortex shedding mechanism were shown to play a role in local flame extinction and the mixing of fuel and oxidiser. Both were furthermore found to roll up the flame front, thereby modulating its instantaneous 'surface area' and local heat release; an in-phase relationship between the flame's 'surface area', flame-averaged mixture fraction and global heat release rate was determined.

\section{ACKNOWLEDGEMENTS}

The authors would like to thank SIEMENS Industrial Turbomachinery Ltd. for their financial support. W. Meier and M. Stöhr, DLR Stuttgart are gratefully acknowledged for providing the experimental data and detailed geometry of the combustor. This work was supported by the EPSRC [Grant no. EP/K026801/1 and EP/R029369/1] through the UK Consortium on Turbulent Reacting Flow (UKCTRF) and used the ARCHER UK National Supercomputing Service (http://www.archer.ac.uk).

\section{DATA AVAILABILITY STATEMENT}

The data that support the findings of this study are available from the corresponding author upon reasonable request.

\section{REFERENCES}

${ }^{1}$ A. H. Lefebvre, Gas Turbine Combustion, 2nd ed. (Taylor \& Francis, 1999).

${ }^{2}$ T. C. Lieuwen and V. Yang, Combustion instabilities in gas turbine engines: operational experience, fundamental mechanisms and modeling (American Institute of Aeronautics and Astronautics, 2005).

${ }^{3}$ J. W. Rayleigh, The Theory of Sound (Macmillan \& Co, London, 1877). 
${ }^{4}$ T. Lieuwen and B. T. Zinn, "The role of equivalence ratio oscillations in driving combustion instabilities in low NOx gas turbines," Symp. Combust. 27, 1809-1816 (1998).

${ }^{5}$ D. L. Straub and G. A. Richards, "Effect of Axial Swirl Vane Location on Combustion Dynamics," in Vol. 2 Coal, Biomass Altern. Fuels; Combust. Fuels; Oil Gas Appl. Cycle Innov. (ASME, 1999) p. V002T02A014.

${ }^{6}$ K. T. Kim, J. G. Lee, B. D. Quay, and D. A. Santavicca, "Response of partially premixed flames to acoustic velocity and equivalence ratio perturbations," Combust. Flame 157, 1731-1744 (2010).

${ }^{7}$ S. Hermeth, G. Staffelbach, L. Y. M. Gicquel, and T. J. Poinsot, "LES evaluation of the effects of equivalence ratio fluctuations on the dynamic flame response in a real gas turbine combustion chamber," Proc. Combust. Inst. 34, 3165-3173 (2013).

${ }^{8}$ B. Ćosić, S. Terhaar, J. P. Moeck, and C. O. Paschereit, "Response of a swirl-stabilized flame to simultaneous perturbations in equivalence ratio and velocity at high oscillation amplitudes," Combust. Flame 162, 1046-1062 (2015).

${ }^{9}$ M. Stöhr, Z. Yin, and W. Meier, "Interaction between velocity fluctuations and equivalence ratio fluctuations during thermoacoustic oscillations in a partially premixed swirl combustor," Proc. Combust. Inst. 36, 3907-3915 (2017).

${ }^{10}$ S. Candel, D. Durox, T. Schuller, J.-F. Bourgouin, and J. P. Moeck, "Dynamics of swirling flames," Annu. Rev. Fluid Mech. 46, 147-173 (2014).

${ }^{11}$ T. Schuller, D. Durox, and S. Candel, "A unified model for the prediction of laminar flame transfer functions: Comparisons between conical and V-flame dynamics," Combust. Flame 134, 21-34 (2003).

${ }^{12}$ K. T. Kim, J. G. Lee, H. J. Lee, B. D. Quay, and D. A. Santavicca, "Characterization of Forced Flame Response of Swirl-Stabilized Turbulent Lean-Premixed Flames in a Gas Turbine Combustor," J. Eng. Gas Turbines Power 132, 041502 (2010).

${ }^{13}$ P. Palies, D. Durox, T. Schuller, and S. Candel, "The combined dynamics of swirler and turbulent premixed swirling flames," Combust. Flame 157, 1698-1717 (2010).

${ }^{14}$ T. J. Poinsot, A. C. Trouve, D. P. Veynante, S. M. Candel, and E. J. Esposito, "Vortexdriven acoustically coupled combustion instabilities," J. Fluid Mech. 177, 265-292 (1987).

${ }^{15}$ J. D. Sterling and E. E. Zukoski, "Longitudinal mode combustion instabilities in a dump combustor," in 25th AIAA Aerosp. Sci. Meet. (American Institute of Aeronautics and Astronautics, 1987) p. 0220. 
${ }^{16}$ K. H. Yu, A. Trouvé, and J. W. Daily, "Low-frequency pressure oscillations in a model ramjet combustor," J. Fluid Mech. 232, 47-72 (1991).

${ }^{17}$ K. K. Venkataraman, L. H. Preston, D. W. Simons, B. J. Lee, J. G. Lee, and D. A. Santavicca, "Mechanism of Combustion Instability in a Lean Premixed Dump Combustor," J. Propuls. Power 15, 909-918 (1999).

${ }^{18}$ C. O. Paschereit, E. Gutmark, and W. Weisenstein, "Excitation of thermoacoustic instabilities by interaction of acoustics and unstable swirling flow," AIAA J. 38, 1025-1034 (2000).

${ }^{19} \mathrm{~N}$. Syred, "A review of oscillation mechanisms and the role of the precessing vortex core (PVC) in swirl combustion systems," Prog. Energy Combust. Sci. 32, 93-161 (2006).

${ }^{20}$ S. Wang, V. Yang, G. Hsiao, S.-Y. Hsieh, and H. C. Mongia, "Large-eddy simulations of gas-turbine swirl injector flow dynamics," J. Fluid Mech. 583, 99 (2007).

${ }^{21}$ J. P. Moeck, J. F. Bourgouin, D. Durox, T. Schuller, and S. Candel, "Nonlinear interaction between a precessing vortex core and acoustic oscillations in a turbulent swirling flame," Combust. Flame 159, 2650-2668 (2012).

${ }^{22}$ F. Lückoff, T. L. Kaiser, C. O. Paschereit, and K. Oberleithner, "Mean field coupling mechanisms explaining the impact of the precessing vortex core on the flame transfer function," Combustion and Flame 223, 254-266 (2021).

${ }^{23}$ S. Hermeth, G. Staffelbach, L. Y. M. Gicquel, V. Anisimov, C. Cirigliano, and T. J. Poinsot, "Bi-stable swirled flames and influence on flame transfer functions," Combust. Flame 161, 184-196 (2014).

${ }^{24}$ A. Schönborn, P. Sayad, and J. Klingmann, "Influence of precessing vortex core on flame flashback in swirling hydrogen flames," Int. J. Hydrogen Energy 39, 20233-20241 (2014).

${ }^{25}$ D. Froud, T. O'Doherty, and N. Syred, "Phase averaging of the precessing vortex core in a swirl burner under piloted and premixed combustion conditions," Combust. Flame 100, 2-11 (1995).

${ }^{26}$ A. P. Dowling and A. S. Morgans, "Feedback control of combustion oscillations," Annu. Rev. Fluid Mech. 37, 151-182 (2005).

${ }^{27}$ Y. Huang and V. Yang, "Dynamics and stability of lean-premixed swirl-stabilized combustion," Prog. Energy Combust. Sci. 35, 293-364 (2009).

${ }^{28}$ T. J. Poinsot, "Prediction and control of combustion instabilities in real engines," Proc. Combust. Inst. 36, 1-28 (2017). 
${ }^{29}$ C. Angelberger, D. Veynante, and F. Egolfopoulos, "LES of chemical and acoustic forcing of a premixed dump combustor," Flow, Turbul. Combust. 65, 205-222 (2000).

${ }^{30}$ W. Polifke, A. Poncet, C. O. Paschereit, and K. Döbbeling, "Reconstruction of acoustic transfer matrices by instationary computational fluid dynamics," J. Sound Vib. 245, 483-510 (2001).

${ }^{31}$ A. Giauque, L. Selle, L. Gicquel, T. J. Poinsot, H. Buechner, P. Kaufmann, and W. Krebs, "System identification of a large-scale swirled partially premixed combustor using LES and measurements," J. Turbul. 6, 1-20 (2005).

${ }^{32}$ N. Noiray, D. Durox, T. Schuller, and S. Candel, "A unified framework for nonlinear combustion instability analysis based on the flame describing function," J. Fluid Mech. 615, 139-167 (2008).

${ }^{33}$ X. Han, J. Li, and A. S. Morgans, "Prediction of combustion instability limit cycle oscillations by combining flame describing function simulations with a thermoacoustic network model," Combust. Flame 162, 3632-3647 (2015).

${ }^{34}$ G. J. Bloxsidge, A. P. Dowling, and P. J. Langhorne, "Reheat buzz: an acoustically coupled combustion instability. Part 2. Theory," J. Fluid Mech. 193, 445-473 (1988).

${ }^{35}$ A. P. Dowling, "Nonlinear self-excited oscillations of a ducted flame," J. Fluid Mech. 346, 271-290 (1997).

${ }^{36}$ G. Erlebacher, M. Y. Hussaini, C. G. Speziale, and T. A. Zang, "Toward the large-eddy simulation of compressible turbulent flows," J. Fluid Mech. 238, 155-185 (1992).

${ }^{37}$ C. Martin, L. Benoit, F. Nicoud, and T. Poinsot, "Analysis of acoustic energy and modes in a turbulent swirled combustor," in Cent. Turbul. Res. Proc. Summer Progr. (2004) pp. 377-394.

${ }^{38}$ S. Roux, G. Lartigue, T. J. Poinsot, U. Meier, and C. Bérat, "Studies of mean and unsteady flow in a swirled combustor using experiments, acoustic analysis, and large eddy simulations," Combust. Flame 141, 40-54 (2005).

${ }^{39}$ P. Schmitt, T. J. Poinsot, B. Schuermans, and K. P. Geigle, "Large-eddy simulation and experimental study of heat transfer, nitric oxide emissions and combustion instability in a swirled turbulent high-pressure burner," J. Fluid Mech. 570, 17-46 (2007).

${ }^{40}$ G. Staffelbach, L. Y. M. Gicquel, G. Boudier, and T. J. Poinsot, "Large Eddy Simulation of self excited azimuthal modes in annular combustors," Proc. Combust. Inst. 32, 29092916 (2009). 
${ }^{41}$ B. Franzelli, E. Riber, L. Y. M. Gicquel, and T. J. Poinsot, "Large Eddy Simulation of combustion instabilities in a lean partially premixed swirled flame," Combust. Flame 159, 621-637 (2012).

${ }^{42}$ S. Tachibana, K. Saito, T. Yamamoto, M. Makida, T. Kitano, and R. Kurose, "Experimental and numerical investigation of thermo-acoustic instability in a liquid-fuel aeroengine combustor at elevated pressure: Validity of large-eddy simulation of spray combustion," Combust. Flame 162, 2621-2637 (2015).

${ }^{43}$ J. M. Lourier, M. Stöhr, B. Noll, S. Werner, and A. Fiolitakis, "Scale Adaptive Simulation of a thermoacoustic instability in a partially premixed lean swirl combustor," Combust. Flame 183, 343-357 (2017).

${ }^{44}$ Z. X. Chen, I. Langella, N. Swaminathan, M. Stöhr, W. Meier, and H. Kolla, "Large Eddy Simulation of a dual swirl gas turbine combustor: Flame/flow structures and stabilisation under thermoacoustically stable and unstable conditions," Combust. Flame 203, 279-300 (2019).

${ }^{45}$ D. Noh, E. Karlis, S. Navarro-Martinez, Y. Hardalupas, A. M. K. P. Taylor, D. Fredrich, and W. P. Jones, "Azimuthally-driven subharmonic thermoacoustic instabilities in a swirlstabilised combustor," Proc. Combust. Inst. 37, 5333-5341 (2019).

${ }^{46}$ O. Schulz, U. Doll, D. Ebi, J. Droujko, C. Bourquard, and N. Noiray, "Thermoacoustic instability in a sequential combustor: Large eddy simulation and experiments," Proc. Combust. Inst. 37, 5325-5332 (2019).

${ }^{47}$ E. L. Schiavo, D. Laera, E. Riber, L. Gicquel, and T. Poinsot, "Effects of liquid fuel/wall interaction on thermoacoustic instabilities in swirling spray flames," Combust. Flame 219, 86-101 (2020).

${ }^{48}$ W. Meier, P. Weigand, X. R. Duan, and R. Giezendanner-Thoben, "Detailed characterization of the dynamics of thermoacoustic pulsations in a lean premixed swirl flame," Combust. Flame 150, 2-26 (2007).

${ }^{49}$ I. Boxx, C. M. Arndt, C. D. Carter, and W. Meier, "High-speed laser diagnostics for the study of flame dynamics in a lean premixed gas turbine model combustor," Exp. Fluids 52, 555-567 (2012).

${ }^{50}$ A. M. Steinberg, C. M. Arndt, and W. Meier, "Parametric study of vortex structures and their dynamics in swirl-stabilized combustion," Proc. Combust. Inst. 34, 3117-3125 (2013). 
${ }^{51}$ V. Caux-Brisebois, A. M. Steinberg, C. M. Arndt, and W. Meier, "Thermo-acoustic velocity coupling in a swirl stabilized gas turbine model combustor," Combust. Flame 161, 3166-3180 (2014).

${ }^{52}$ R. Zhang, I. Boxx, W. Meier, and C. D. Slabaugh, "Coupled interactions of a helical precessing vortex core and the central recirculation bubble in a swirl flame at elevated power density," Combust. Flame 202, 119-131 (2019).

${ }^{53}$ K. Oberleithner, M. Stöhr, S. H. Im, C. M. Arndt, and A. M. Steinberg, "Formation and flame-induced suppression of the precessing vortex core in a swirl combustor: Experiments and linear stability analysis," Combust. Flame 162, 3100-3114 (2015).

${ }^{54}$ Q. An, B. D. Kwong, W. Y.and Geraedts, and A. M. Steinberg, "Coupled dynamics of lift-off and precessing vortex core formation in swirl flames," Combust. Flame 168, 228-239 (2016).

${ }^{55}$ Z. Yin, P. Nau, and W. Meier, "Responses of combustor surface temperature to flame shape transitions in a turbulent bi-stable swirl flame," Exp. Therm. Fluid Sci. 82, 50-57 (2017).

${ }^{56}$ M. Stöhr, K. Oberleithner, M. Sieber, Z. Yin, and W. Meier, "Experimental Study of Transient Mechanisms of Bi-stable Flame Shape Transitions in a Swirl Combustor," J. Eng. Gas Turbines Power 140, 011503 (2018).

${ }^{57}$ Q. An and A. M. Steinberg, "The role of strain rate, local extinction, and hydrodynamic instability on transition between attached and lifted swirl flames," Combust. Flame 199, 267-278 (2019).

${ }^{58}$ G. Bulat, W. P. Jones, and A. J. Marquis, "NO and $\mathrm{CO}$ formation in an industrial gas-turbine combustion chamber using LES with the Eulerian sub-grid PDF method," Combust. Flame 161, 1804-1825 (2014).

${ }^{59}$ D. Fredrich, W. P. Jones, and A. J. Marquis, "Application of the Eulerian subgrid Probability Density Function method in the Large Eddy Simulation of a partially premixed swirl flame," Combust. Sci. Technol. 191, 137-150 (2019).

${ }^{60}$ Y. Gong, W. Jones, and A. Marquis, "Study of a premixed turbulent counter-flow flame with a large eddy simulation method," Flow, Turbulence and Combustion 106, 1379-1398 (2021).

${ }^{61}$ T. Pant, U. Jain, and H. Wang, "Transported pdf modeling of compressible turbulent reactive flows by using the eulerian monte carlo fields method," Journal of Computational 
Physics 425, 109899 (2021).

${ }^{62}$ C. Gong, M. Jangi, X.-S. Bai, J.-H. Liang, and M.-B. Sun, "Large eddy simulation of hydrogen combustion in supersonic flows using an eulerian stochastic fields method," international journal of hydrogen energy 42, 1264-1275 (2017).

${ }^{63}$ Y. P. D. Almeida and S. Navarro-Martinez, "Large Eddy Simulation of a supersonic lifted flame using the Eulerian stochastic fields method," Proc. Combust. Inst. 37, 3693-3701 (2019).

${ }^{64}$ J. Smagorinsky, "General Circulation Experiments with the Primitive Equations," Mon. Weather Rev. 91, 99-164 (1963).

${ }^{65} \mathrm{U}$. Piomelli and J. Liu, "Large-eddy simulation of rotating channel flows using a localized dynamic model," Phys. Fluids 7, 839-848 (1995).

${ }^{66}$ T. J. Poinsot and D. Veynante, Theoretical and Numerical Combustion, 2nd ed. (RT Edwards, Inc., 2005).

${ }^{67}$ F. Gao and E. E. O'Brien, "A large-eddy simulation scheme for turbulent reacting flows," Phys. Fluids A 5, 1282-1284 (1993).

${ }^{68}$ T. Brauner, W. P. Jones, and A. J. Marquis, "LES of the Cambridge Stratified Swirl Burner using a Sub-grid pdf Approach," Flow, Turbul. Combust. 96, 965-985 (2016).

${ }^{69}$ C. Dopazo and E. E. O'Brien, "Functional formulation of nonisothermal turbulent reactive flows," Phys. Fluids 17, 1968-1975 (1974).

${ }^{70}$ W. P. Jones, A. J. Marquis, and V. N. Prasad, "LES of a turbulent premixed swirl burner using the Eulerian stochastic field method," Combust. Flame 159, 3079-3095 (2012).

${ }^{71}$ V. Sabel'nikov and O. Soulard, "Rapidly decorrelating velocity-field model as a tool for solving one-point Fokker-Planck equations for probability density functions of turbulent reactive scalars," Phys. Rev. E 72, 1-22 (2005).

${ }^{72}$ C. Gardiner, Handbook of Stochastic Methods (Springer-Verlag, New York, 1985).

${ }^{73}$ W. P. Jones and S. Navarro-Martinez, "Large eddy simulation of autoignition with a subgrid probability density function method," Combust. Flame 150, 170-187 (2007).

${ }^{74}$ W. P. Jones and V. N. Prasad, "Large Eddy Simulation of the Sandia Flame Series (D-F) using the Eulerian stochastic field method," Combust. Flame 157, 1621-1636 (2010).

${ }^{75}$ D. Fredrich, W. P. Jones, and A. J. Marquis, "Thermo-acoustic instabilities in the preccinsta combustor investigated using a compressible les-pdf approach," Flow, Turbulence and Combustion 106, 1399-1415 (2021). 
${ }^{76}$ P. E. Kloeden and E. Platen, Numerical Solution of Stochastic Differential Equations (Springer-Verlag, 1999).

${ }^{77} \mathrm{~T}$. Lu and C. K. Law, "A criterion based on computational singular perturbation for the identification of quasi steady state species: A reduced mechanism for methane oxidation with NO chemistry," Combust. Flame 154, 761-774 (2008).

${ }^{78}$ T. Readshaw, T. Ding, S. Rigopoulos, and W. Jones, "Modeling of turbulent flames with the large eddy simulation-probability density function (les-pdf) approach, stochastic fields, and artificial neural networks," Physics of Fluids 33, 035154 (2021).

${ }^{79}$ T. J. Poinsot and S. K. Lele, "Boundary conditions for direct simulations of compressible viscous flows," J. Comput. Phys. 101, 104-129 (1992).

${ }^{80}$ C. S. Yoo, Y. Wang, A. Trouvé, and H. G. Im, "Characteristic boundary conditions for direct simulations of turbulent counterflow flames," Combust. Theory Model. 9, 617-646 (2005).

${ }^{81}$ U. Piomelli and E. Balaras, "Wall layer models for large eddy simulation," Annu. Rev. Fluid Mech. 34, 349-374 (2002).

${ }^{82}$ D. Fredrich, W. P. Jones, and A. J. Marquis, "The stochastic fields method applied to a partially premixed swirl flame with wall heat transfer," Combust. Flame 205, 446-456 (2019).

${ }^{83}$ S. Gövert, D. Mira, J. B. W. Kok, M. Vázquez, and G. Houzeaux, "The Effect of Partial Premixing and Heat Loss on the Reacting Flow Field Prediction of a Swirl Stabilized Gas Turbine Model Combustor," Flow, Turbul. Combust. 100, 503-534 (2018).

${ }^{84}$ P. Benard, G. Lartigue, V. Moureau, and R. Mercier, "Large-Eddy Simulation of the leanpremixed PRECCINSTA burner with wall heat loss," Proc. Combust. Inst. 37, 5233-5243 (2019).

${ }^{85}$ J. E. Temme, P. M. Allison, and J. F. Driscoll, "Combustion instability of a lean premixed prevaporized gas turbine combustor studied using phase-averaged PIV," Combust. Flame 161, 958-970 (2014).

${ }^{86}$ Y. Gong, D. Fredrich, A. Marquis, W. Jones, and I. Boxx, "Thermoacoustic instabilities of hydrogen-enriched partially premixed flames in a swirl combustor," in Proc. ASME Turbo Expo 2021 Turbomach. Tech. Conf. Expo. (2021) pp. GT2021-60009.

${ }^{87}$ V. N. Prasad, M. Juddoo, A. Kourmatzis, and A. R. Masri, "Investigation of lifted flame propagation under pulsing conditions using high-speed OH-LIF and LES," Flow, Turbul. 
Combust. 93, 425-437 (2014).

${ }^{88}$ P. Palies, D. Durox, T. Schuller, and S. Candel, "Acoustic-convective mode conversion in an aerofoil cascade," J. Fluid Mech. 672, 545-569 (2011).

${ }^{89}$ N. A. Cumpsty and F. E. Marble, "The interaction of entropy fluctuations with turbine blade rows; a mechanism of turbojet engine noise," Proc. R. Soc. Lond. Ser. A. 357, 323-344 (1977).

${ }^{90}$ M. Sajben and H. Said, "Acoustic-Wave/Blade-Row Interactions Establish Boundary Conditions for Unsteady Inlet Flows," J. Propuls. Power 17, 1090-1099 (2001).

${ }^{91}$ S. Wang and V. Yang, "Unsteady flow evolution in swirl injectors with radial entry. II. External excitations," Phys. Fluids 17 (2005).

${ }^{92}$ T. Komarek and W. Polifke, "Impact of Swirl Fluctuations on the Flame Response of a Perfectly Premixed Swirl Burner," J. Eng. Gas Turbines Power 132, 061503 (2010).

${ }^{93}$ P. Palies, T. Schuller, D. Durox, L. Y. M. Gicquel, and S. Candel, "Acoustically perturbed turbulent premixed swirling flames," Phys. Fluids 23, 037101 (2011).

${ }^{94} \mathrm{P}$. Palies, M. Ilak, and R. Cheng, "Transient and limit cycle combustion dynamics analysis of turbulent premixed swirling flames," J. Fluid Mech. 830, 681-707 (2017).

${ }^{95}$ J. M. Beér and N. A. Chigier, Combustion Aerodynamics, reprint ed. (Krieger Publishing Company, 1983).

${ }^{96}$ C. Duwig and L. Fuchs, "Large eddy simulation of vortex breakdown/flame interaction," Phys. Fluids 19 (2007).

${ }^{97}$ M. Sieber, C. O. Paschereit, and K. Oberleithner, "Spectral proper orthogonal decomposition,” J. Fluid Mech. 792, 798-828 (2016).

${ }^{98}$ O. Lucca-Negro and T. O'Doherty, "Vortex breakdown: A review," Prog. Energy Combust. Sci. 27, 431-481 (2001).

${ }^{99}$ S. Terhaar, K. Oberleithner, and C. O. Paschereit, "Key parameters governing the precessing vortex core in reacting flows: An experimental and analytical study," Proc. Combust. Inst. 35, 3347-3354 (2015).

${ }^{100}$ E. Karlis, Y. Hardalupas, and A. M. Taylor, "Effects of inert fuel diluents on the dynamic state of a thermoacosutically unstable gas turbine combustor," in AIAA Scitech 2019 Forum (2019) pp. 1-20.

${ }^{101}$ M. A. Ahmed and M. Birouk, "Effect of fuel nozzle geometry and airflow swirl on the coherent structures of partially premixed methane flame under flashback conditions," Exp. 
Therm. Fluid Sci. 99, 304-314 (2018).

${ }^{102}$ E. Karlis, Y. Liu, Y. Hardalupas, and A. M. Taylor, "Extinction strain rate suppression of the precessing vortex core in a swirl stabilised combustor and consequences for thermoacoustic oscillations," Combust. Flame 211, 229-252 (2020).

${ }^{103}$ C. Fureby, "A computational study of combustion instabilities due to vortex shedding," Proc. Combust. Inst. 28, 783-791 (2000).

${ }^{104}$ R. Balachandran, B. O. Ayoola, C. F. Kaminski, A. P. Dowling, and E. Mastorakos, "Experimental investigation of the nonlinear response of turbulent premixed flames to imposed inlet velocity oscillations," Combust. Flame 143, 37-55 (2005).

${ }^{105}$ A. M. Steinberg, I. Boxx, C. M. Arndt, J. H. Frank, and W. Meier, "Experimental study of flame-hole reignition mechanisms in a turbulent non-premixed jet flame using sustained multi-kHz PIV and crossed-plane OH PLIF," Proc. Combust. Inst. 33, 1663-1672 (2011).

${ }^{106}$ A. F. Ghoniem, S. Park, A. Wachsman, A. Annaswamy, D. Wee, and H. Murat Altay, "Mechanism of combustion dynamics in a backward-facing step stabilized premixed flame," Proc. Combust. Inst. 30, 1783-1790 (2005).

${ }^{107}$ M. Stöhr, I. Boxx, C. Carter, and W. Meier, "Dynamics of lean blowout of a swirlstabilized flame in a gas turbine model combustor," Proc. Combust. Inst. 33, 2953-2960 (2011).

${ }^{108}$ A. M. Steinberg, I. Boxx, M. Stöhr, C. D. Carter, and W. Meier, "Flow-flame interactions causing acoustically coupled heat release fluctuations in a thermo-acoustically unstable gas turbine model combustor," Combust. Flame 157, 2250-2266 (2010).

${ }^{109}$ R. Ranjan, D. F. Ebi, and N. T. Clemens, "Role of inertial forces in flame-flow interaction during premixed swirl flame flashback," Proc. Combust. Inst. 37, 5155-5162 (2019).

${ }^{110}$ P. A. Monkewitz, "A note on vortex shedding from axisymmetric bluff bodies," J. Fluid Mech. 192, 561-575 (1988).

${ }^{111}$ V. S. Acharya, D.-H. Shin, and T. Lieuwen, "Premixed flames excited by helical disturbances: Flame wrinkling and heat release oscillations," Journal of Propulsion and Power 29, 1282-1291 (2013).

${ }^{112}$ Y. Ju, H. Guo, K. Maruta, and F. Liu, "On the extinction limit and flammability limit of non-adiabatic stretched methane-air premixed flames," J. Fluid Mech. 342, 315-334 (1997). 


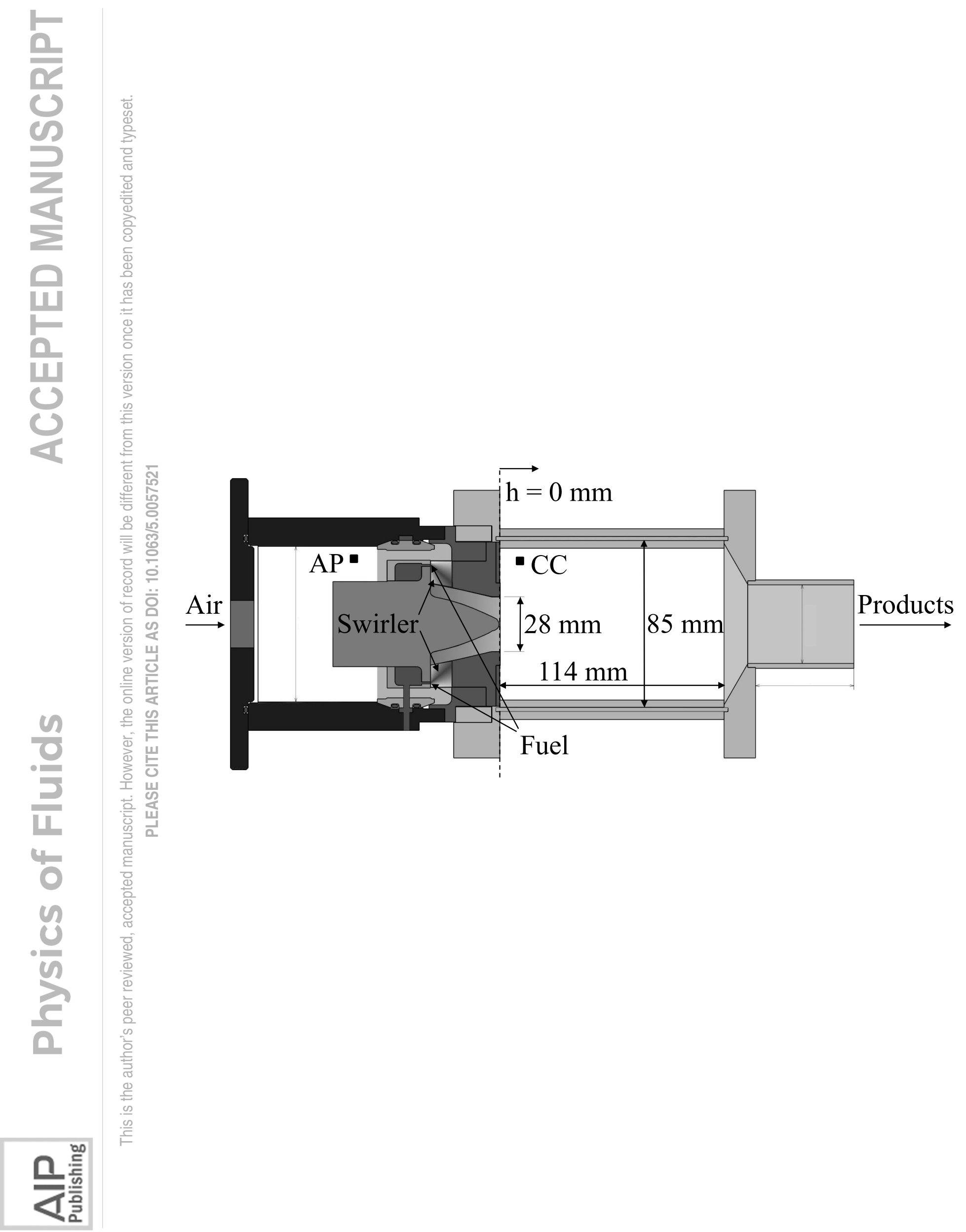




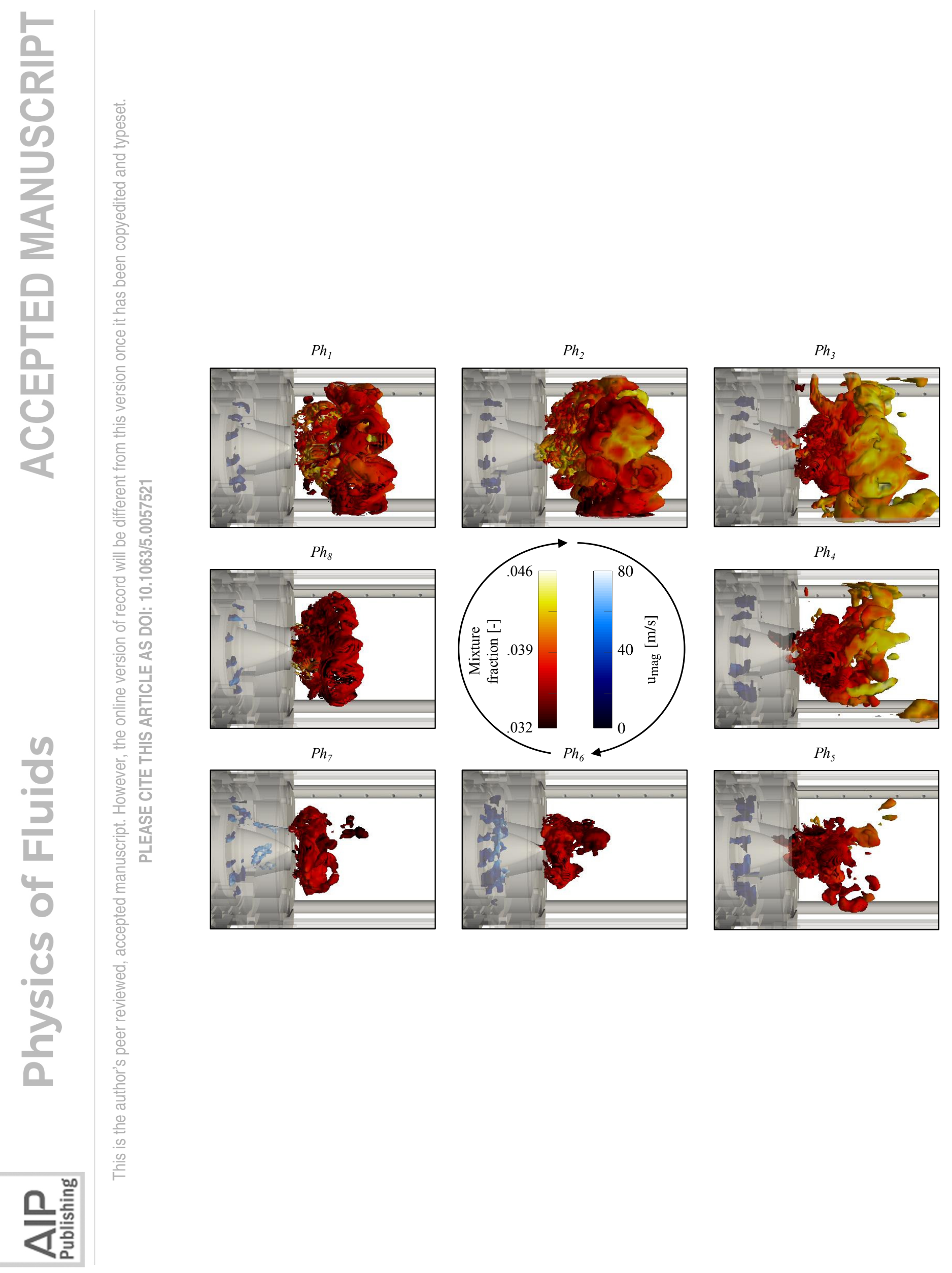




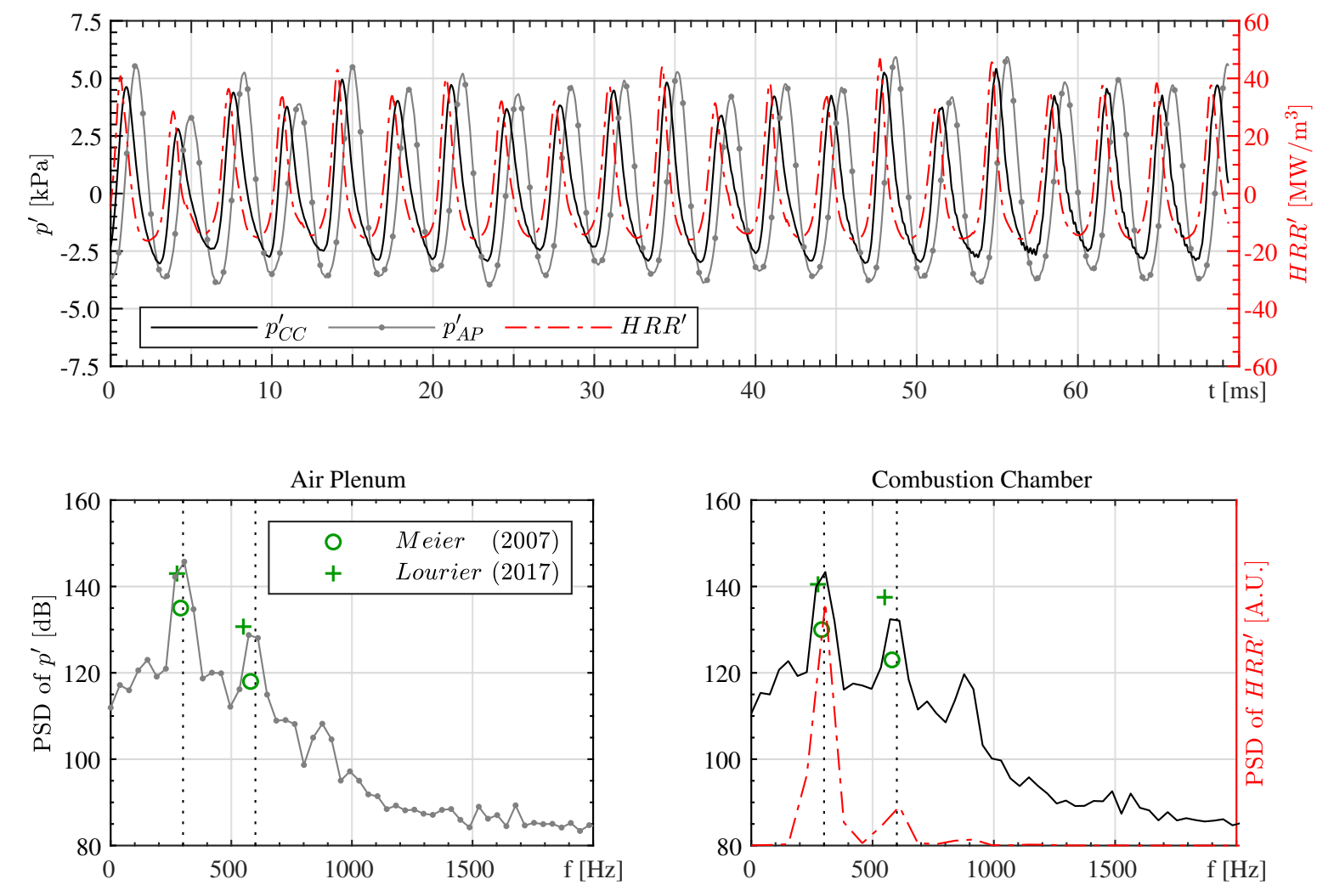




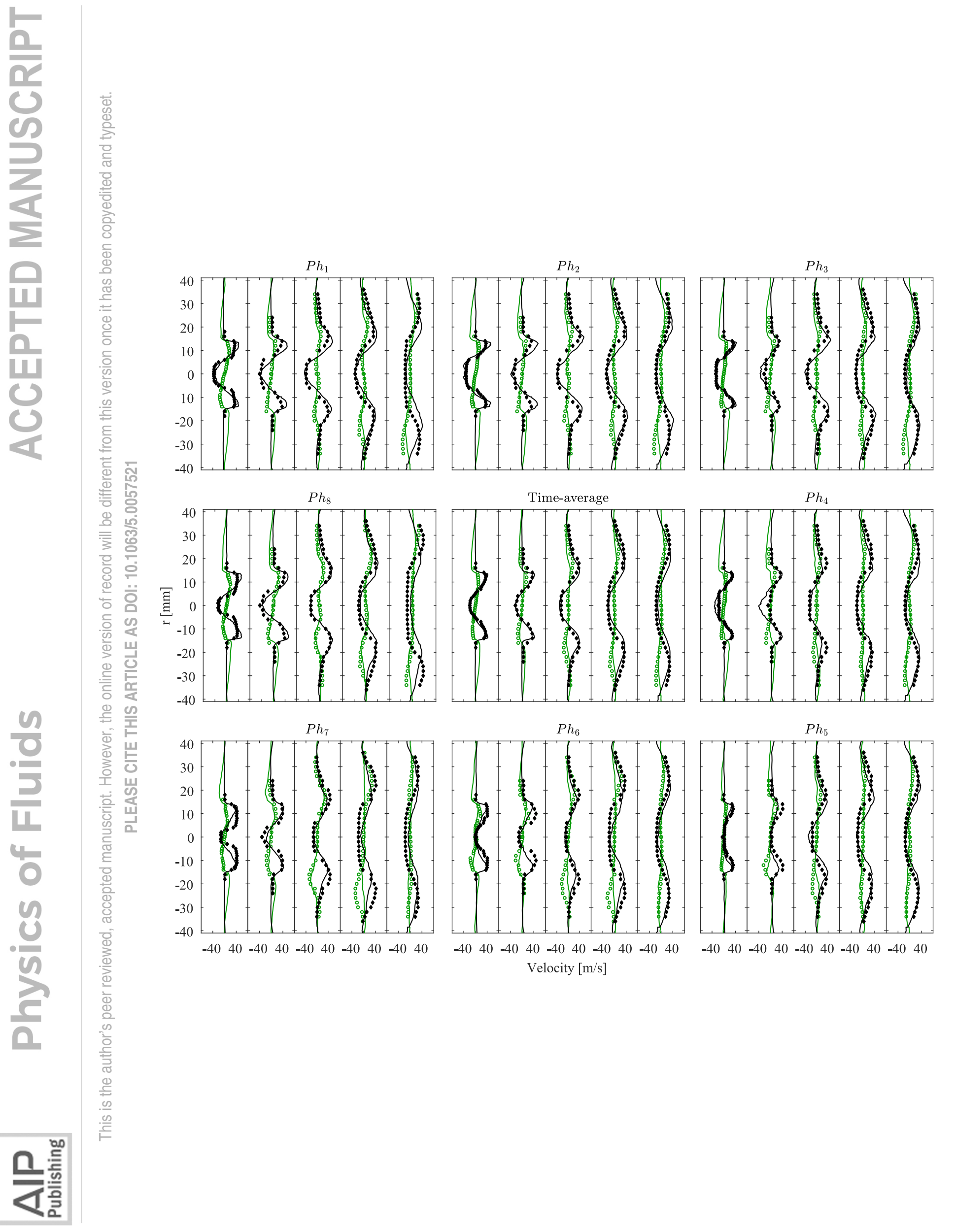



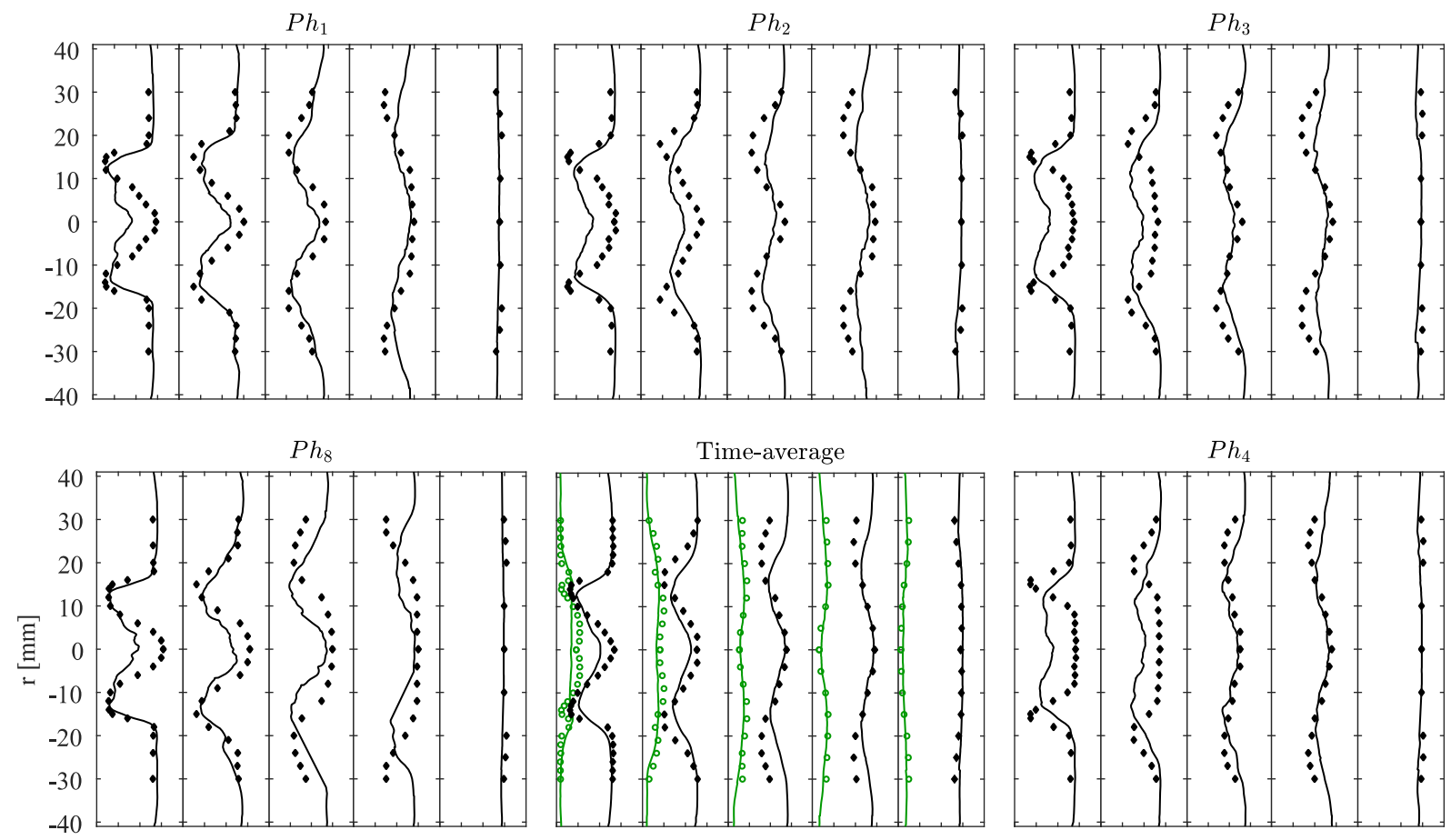

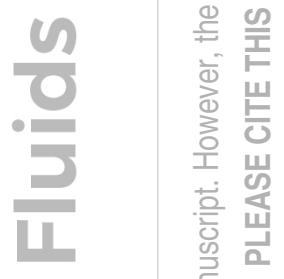
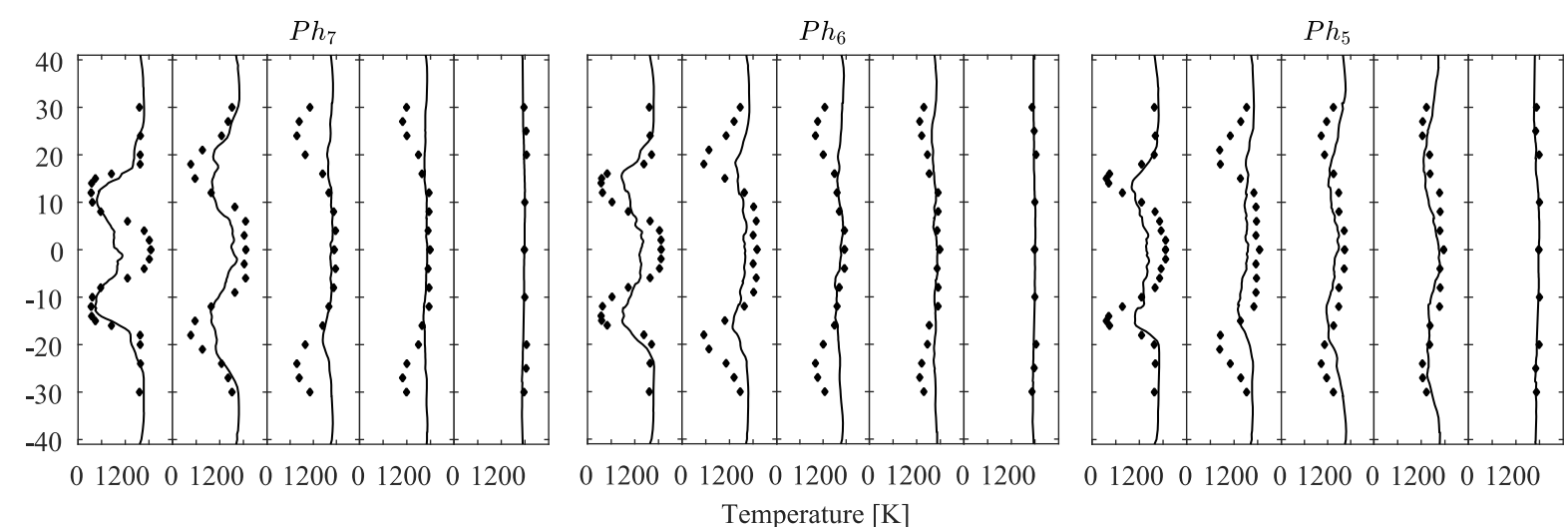

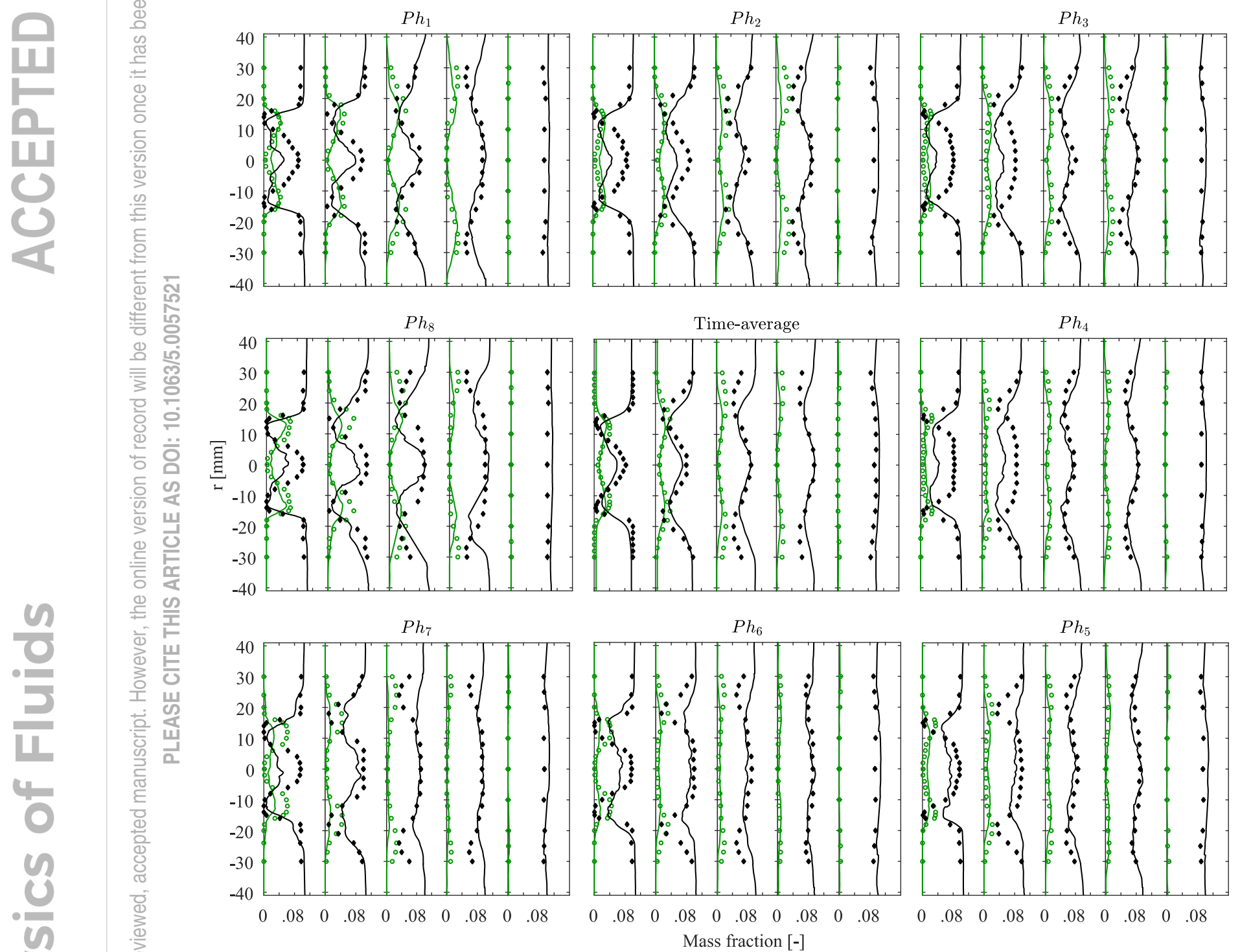


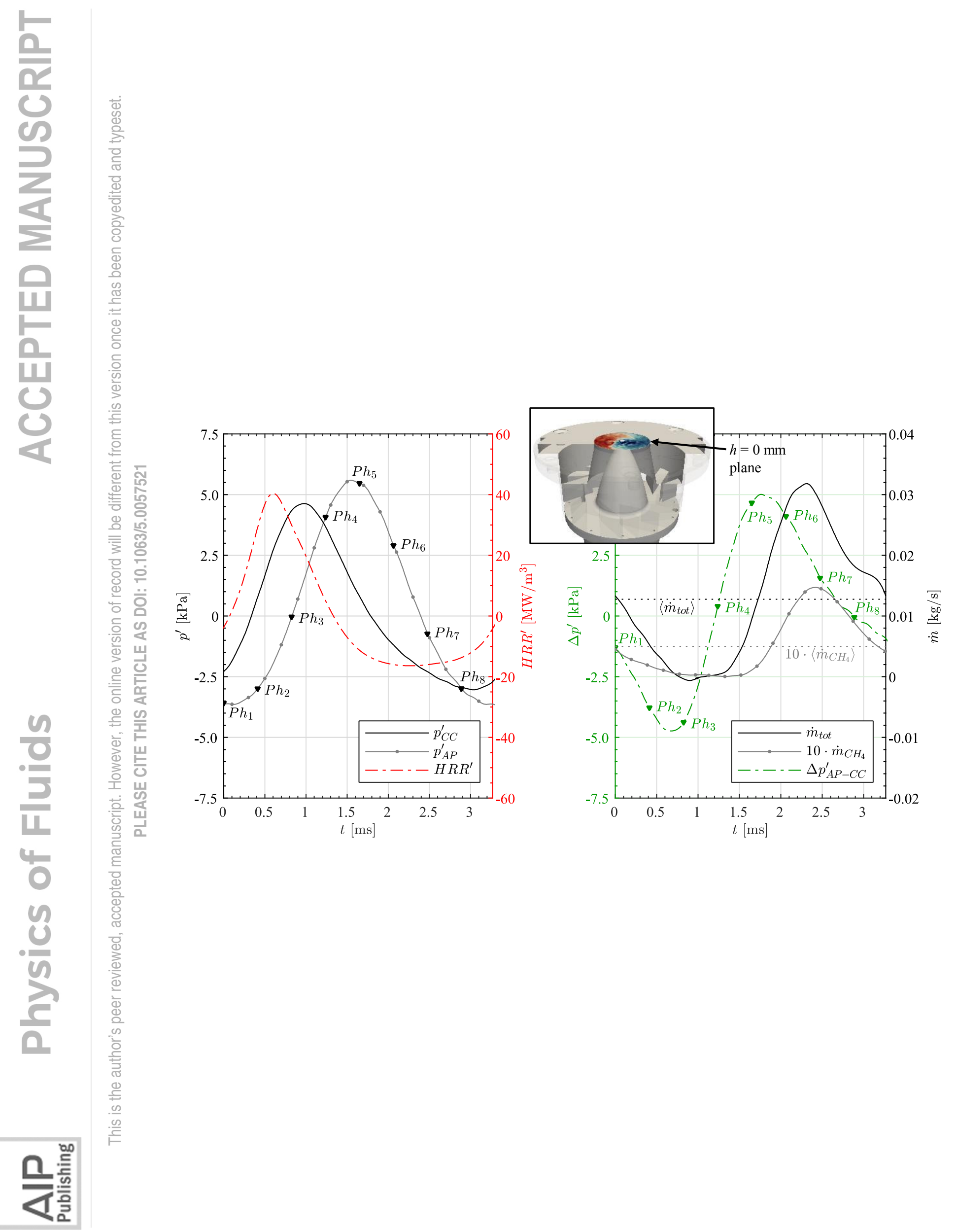




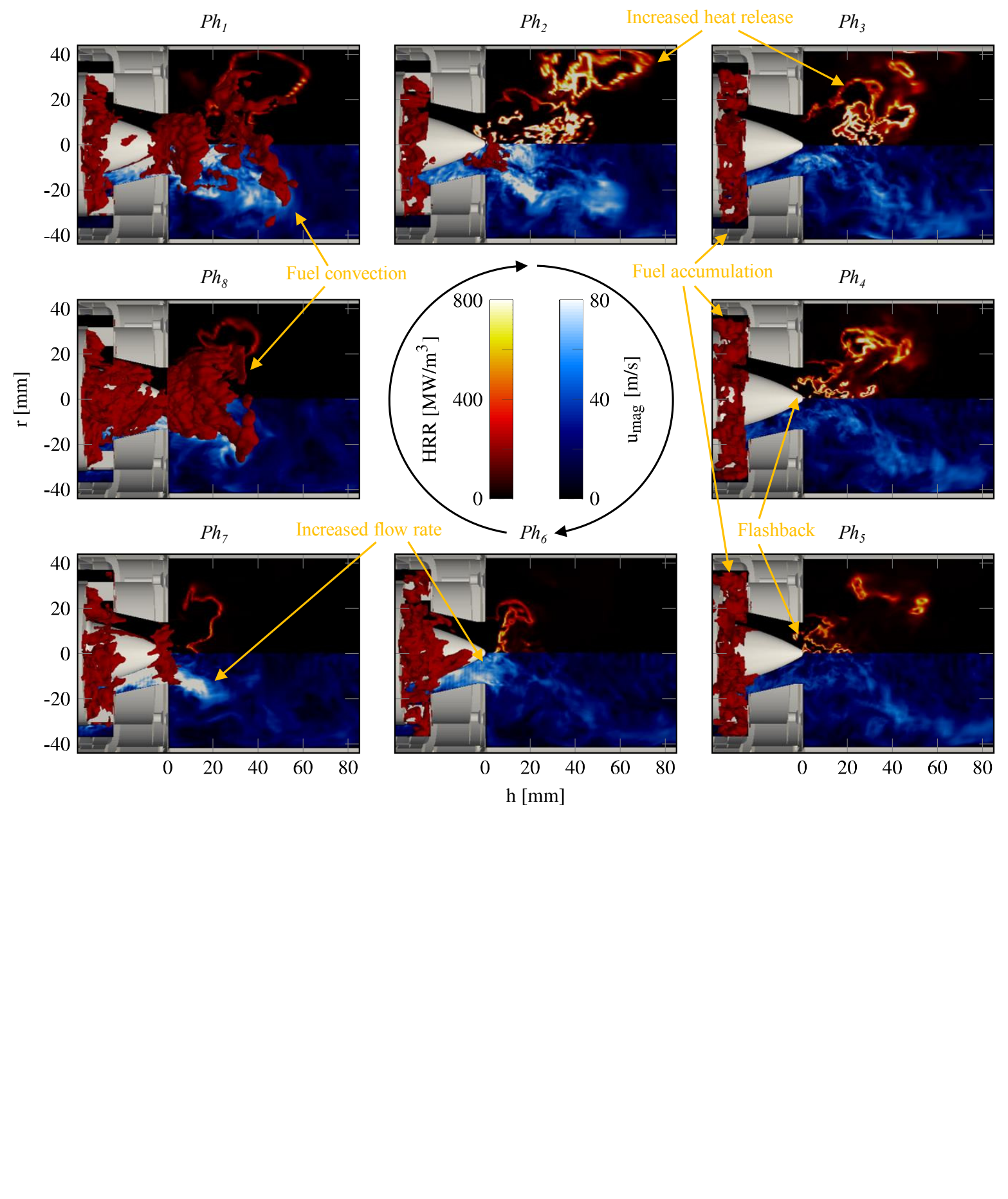




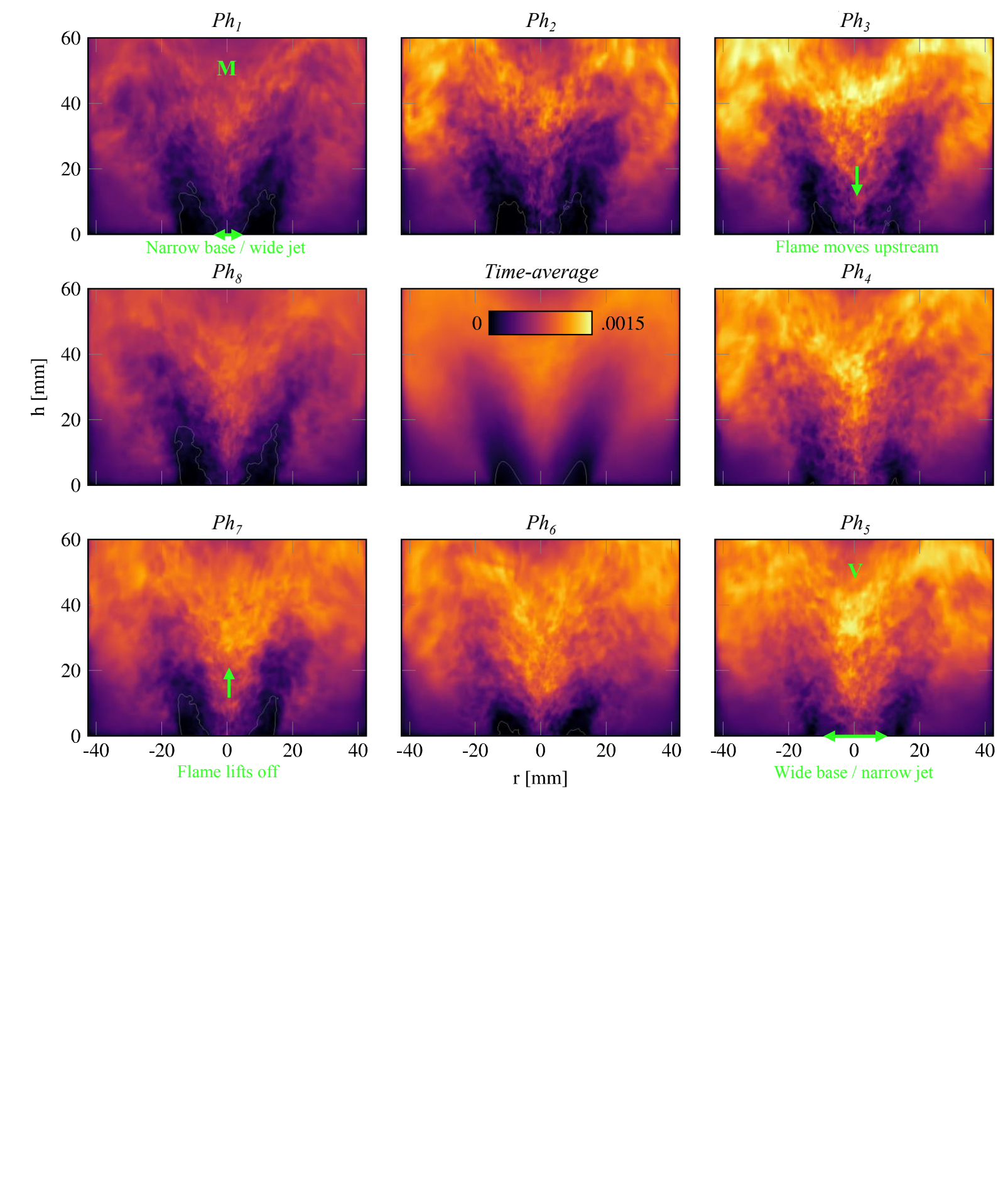




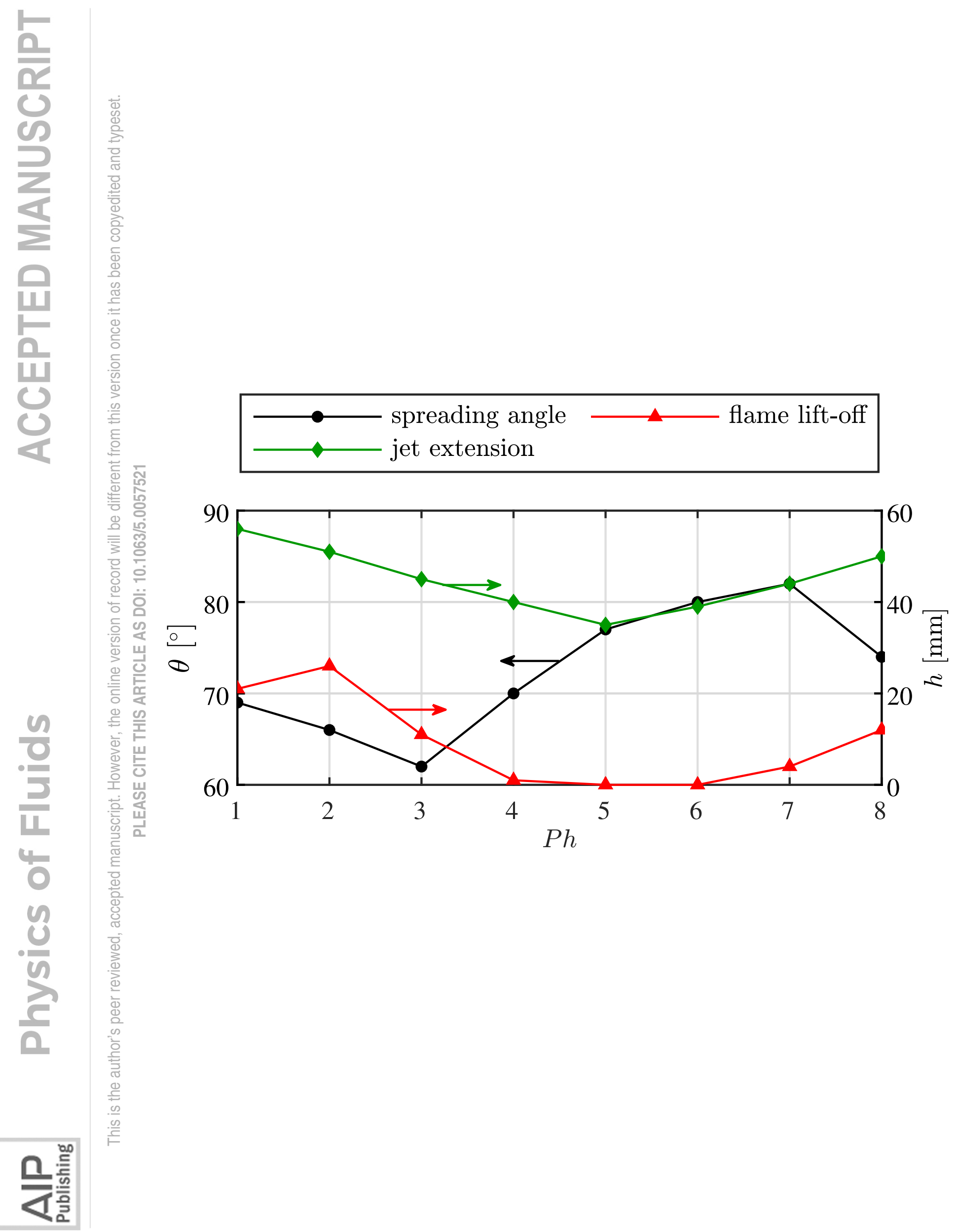




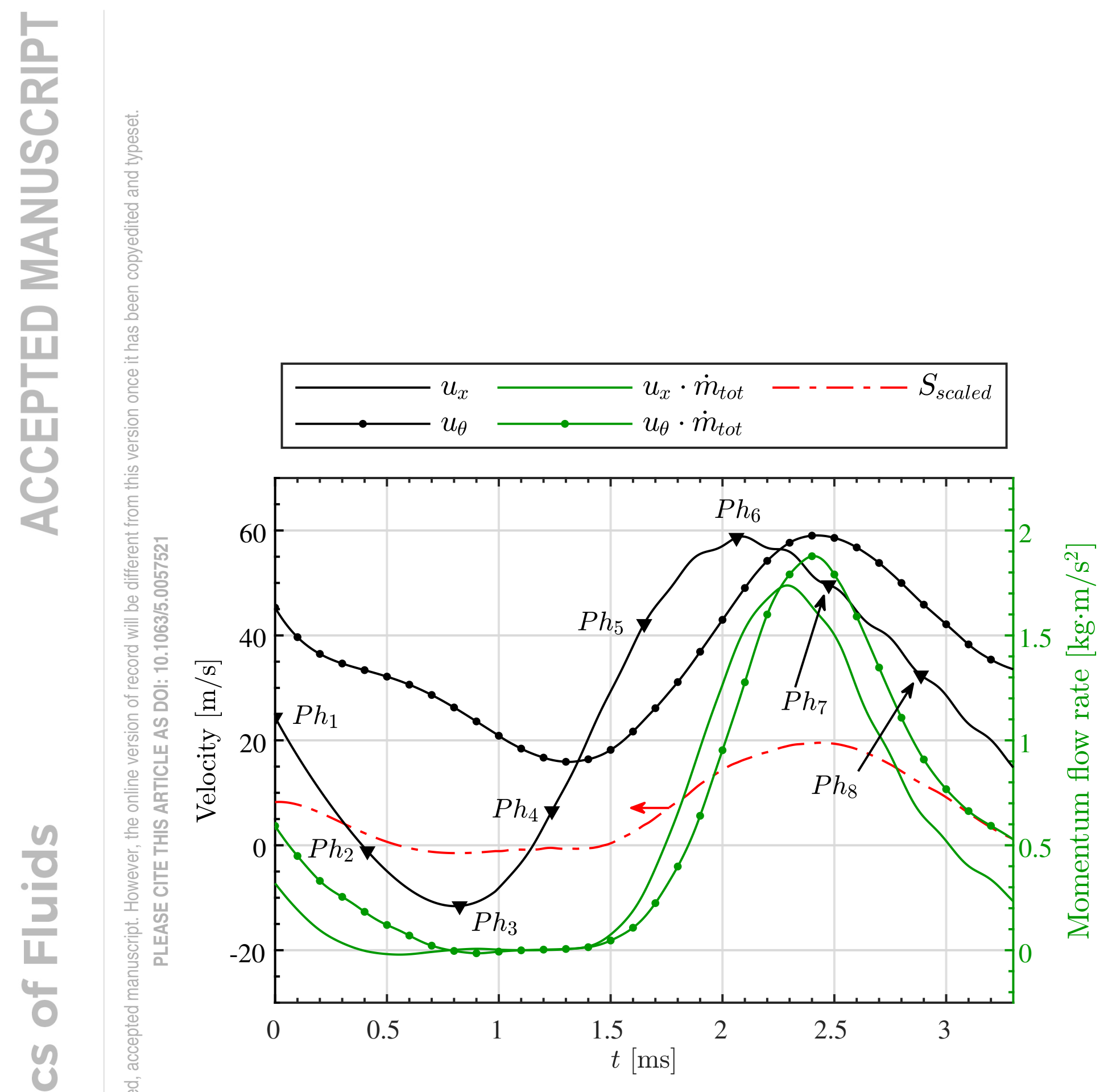




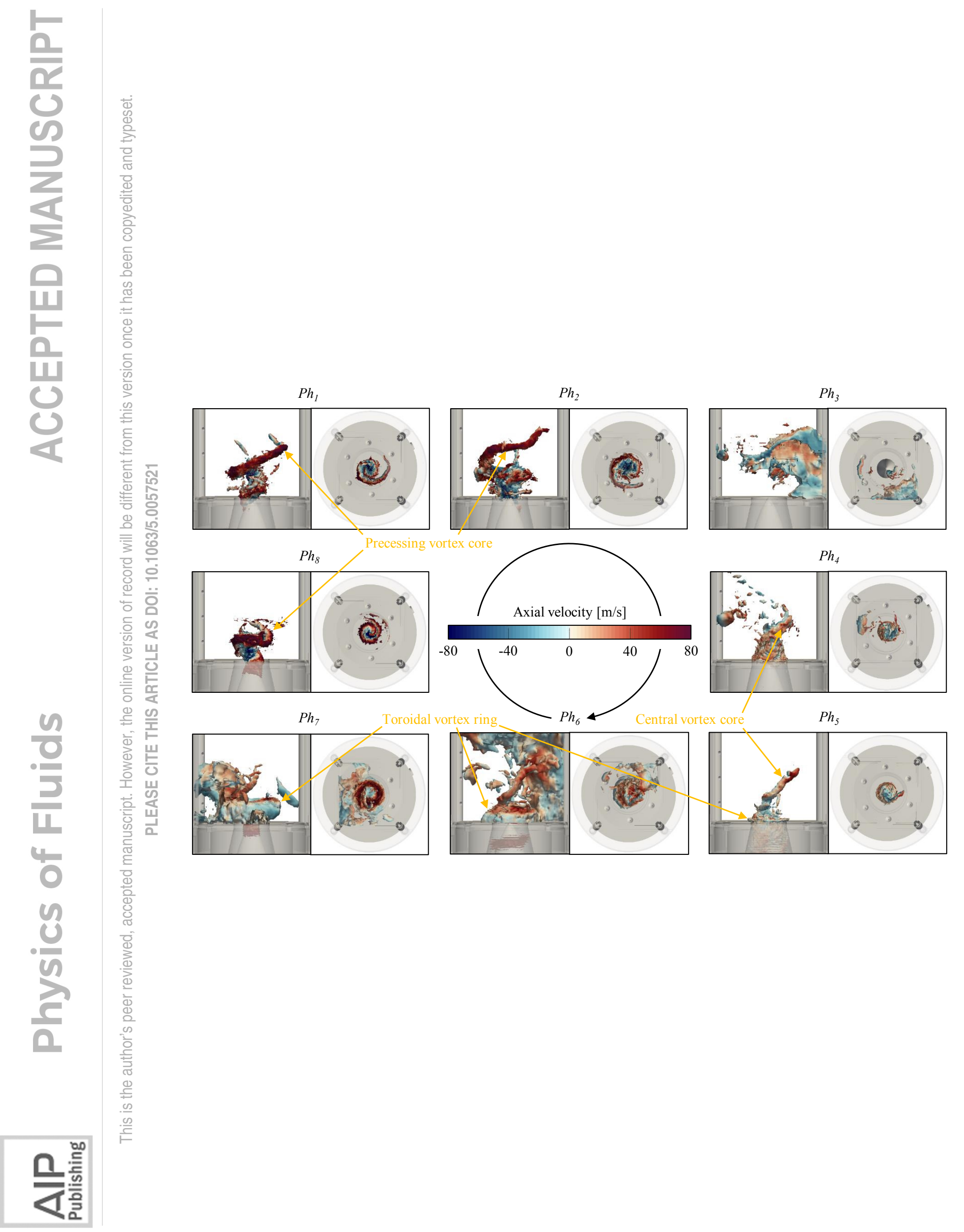




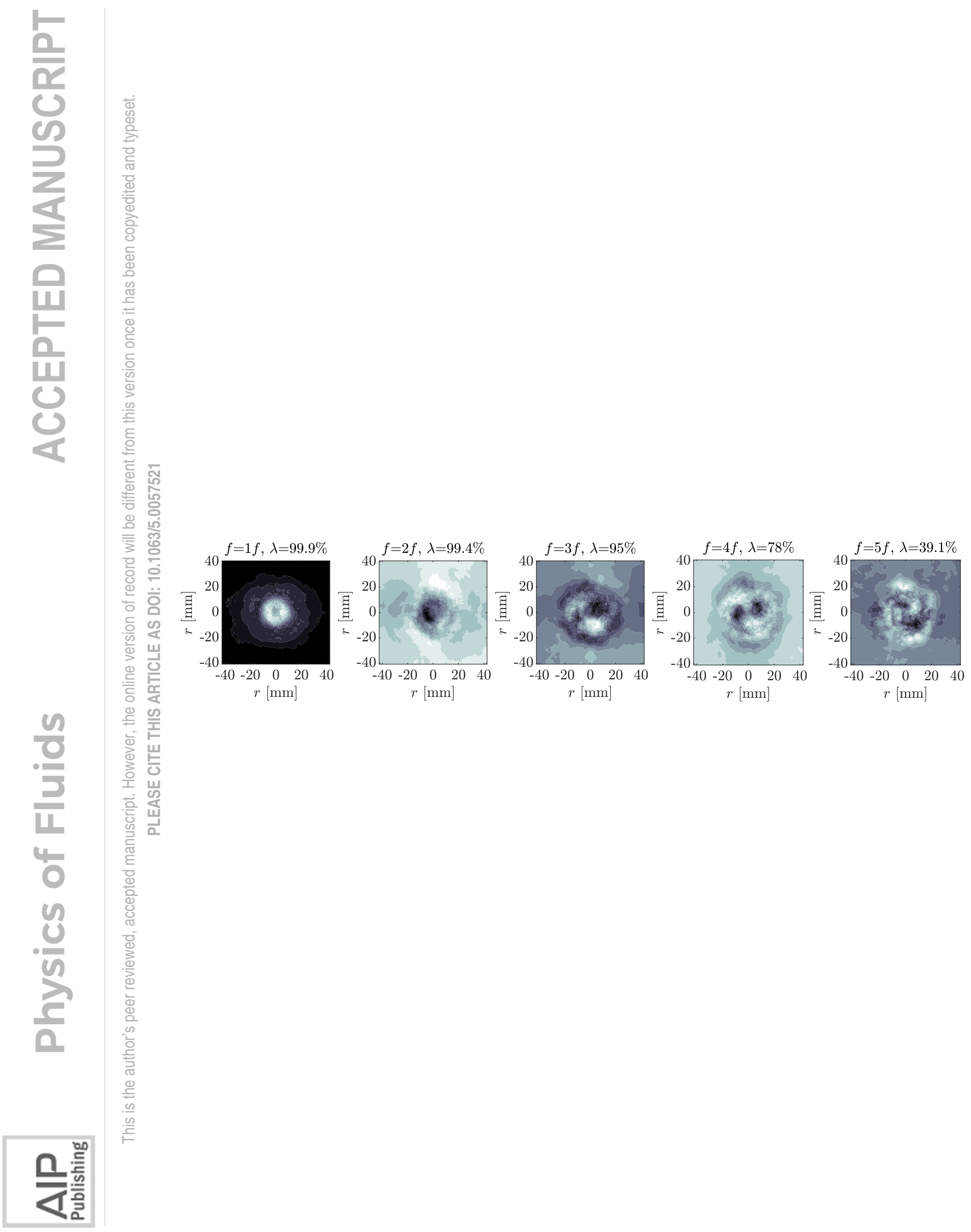




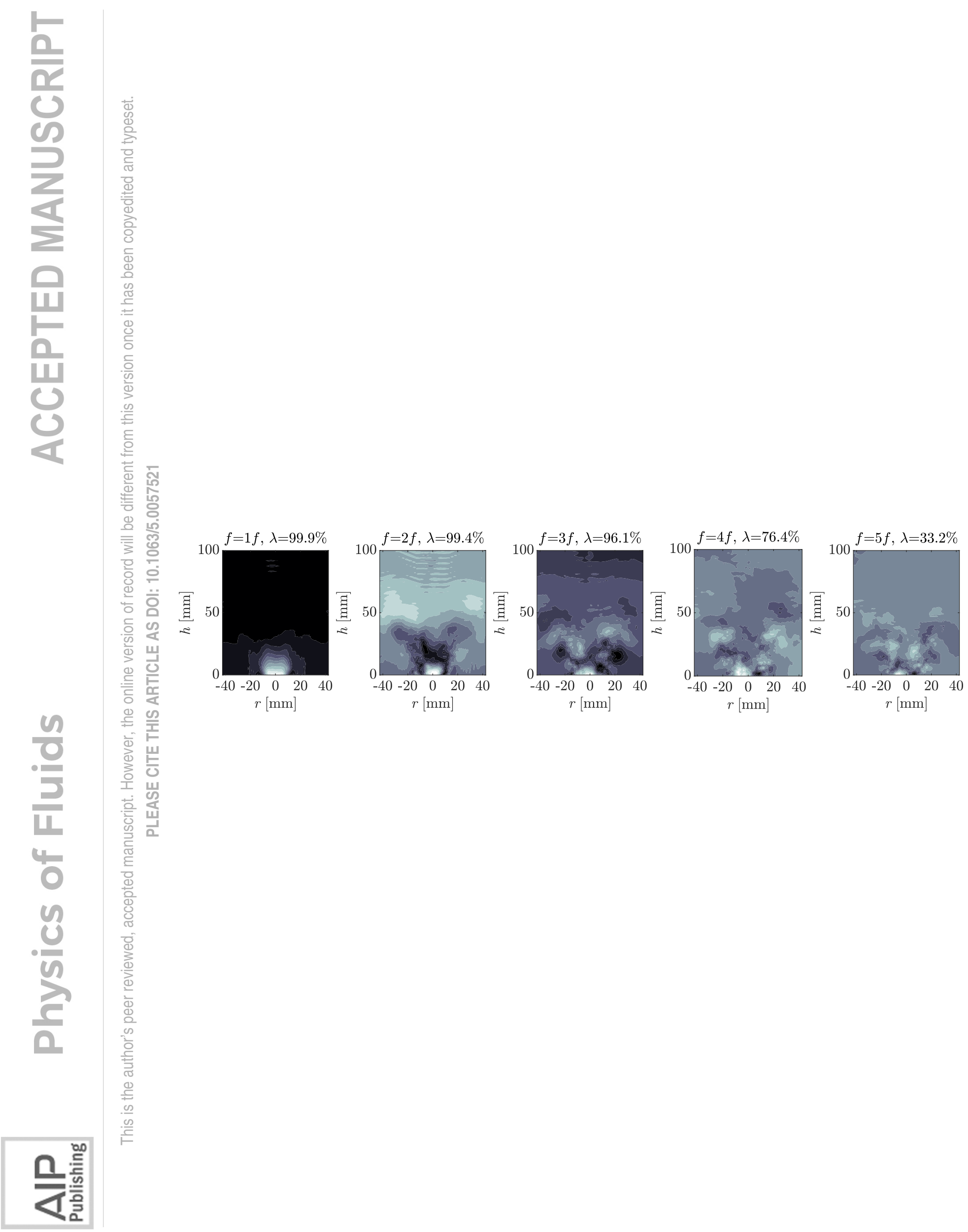




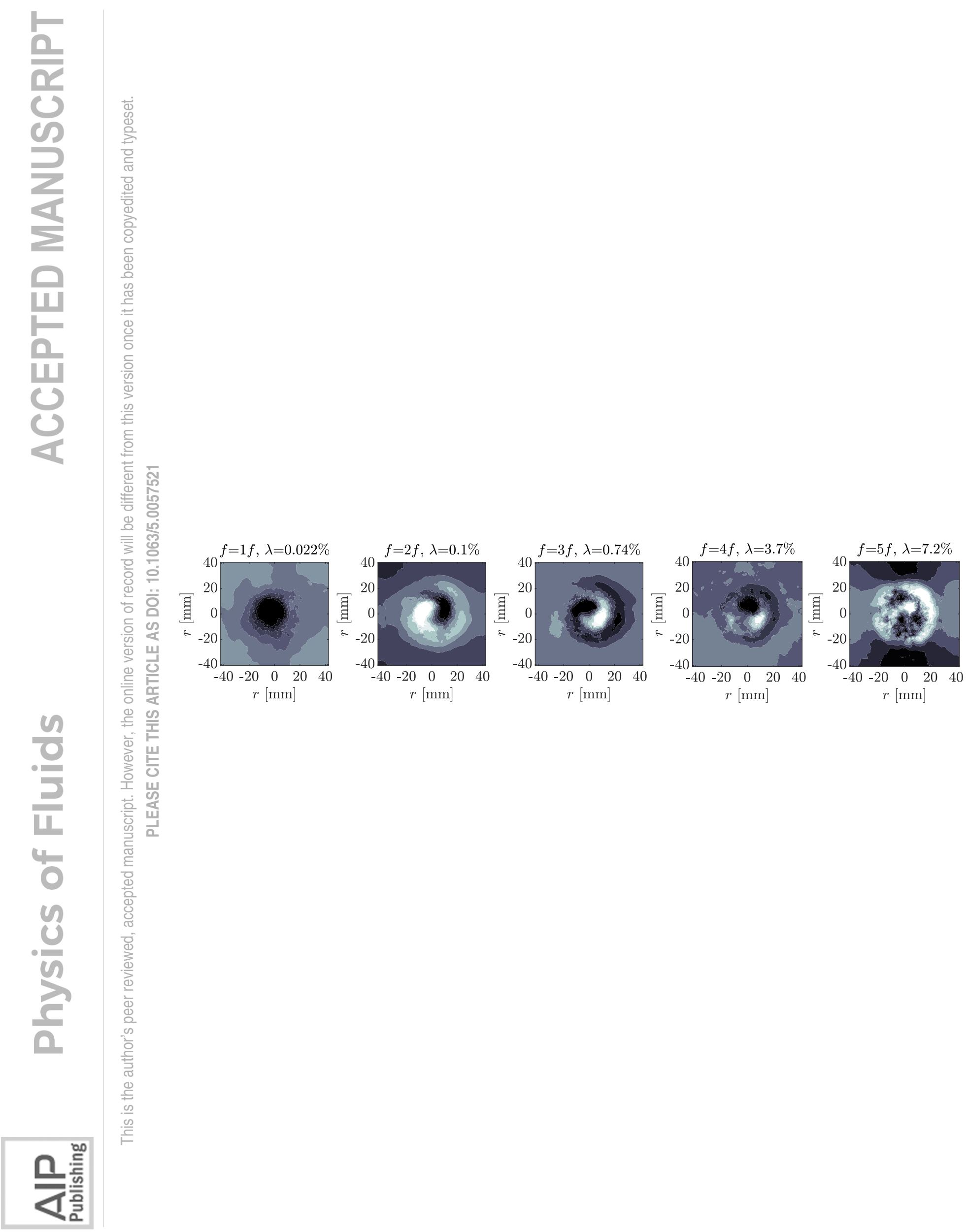




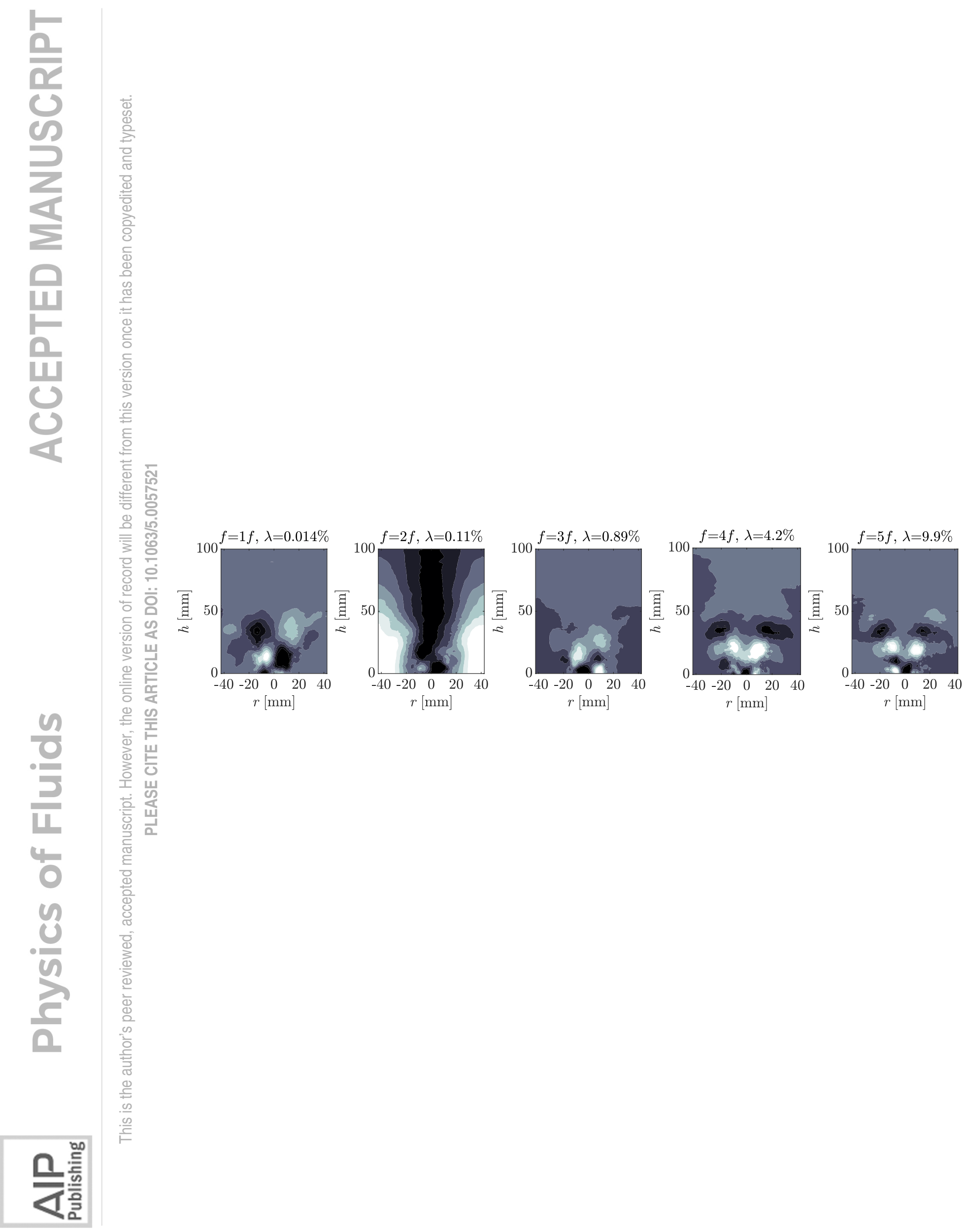




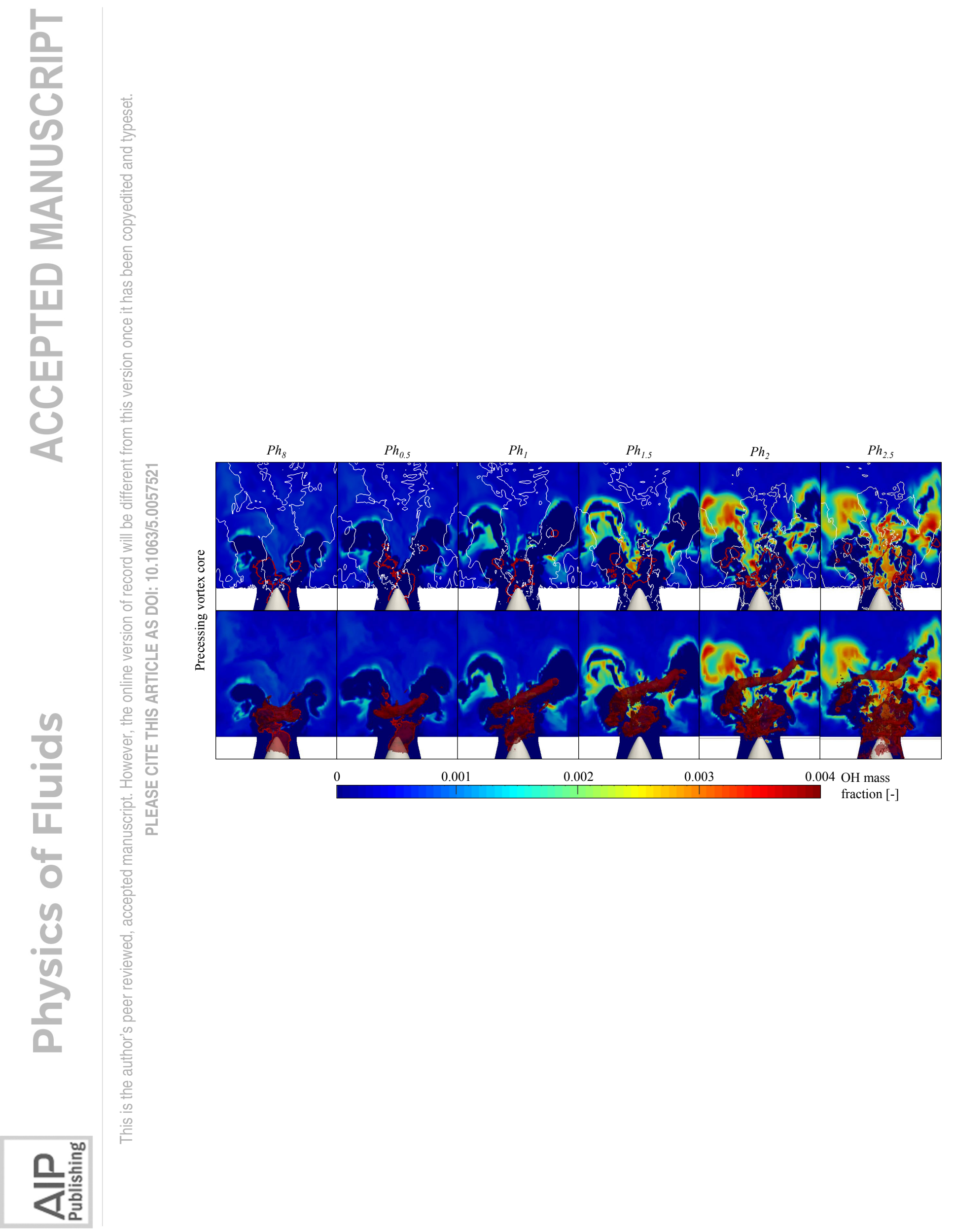




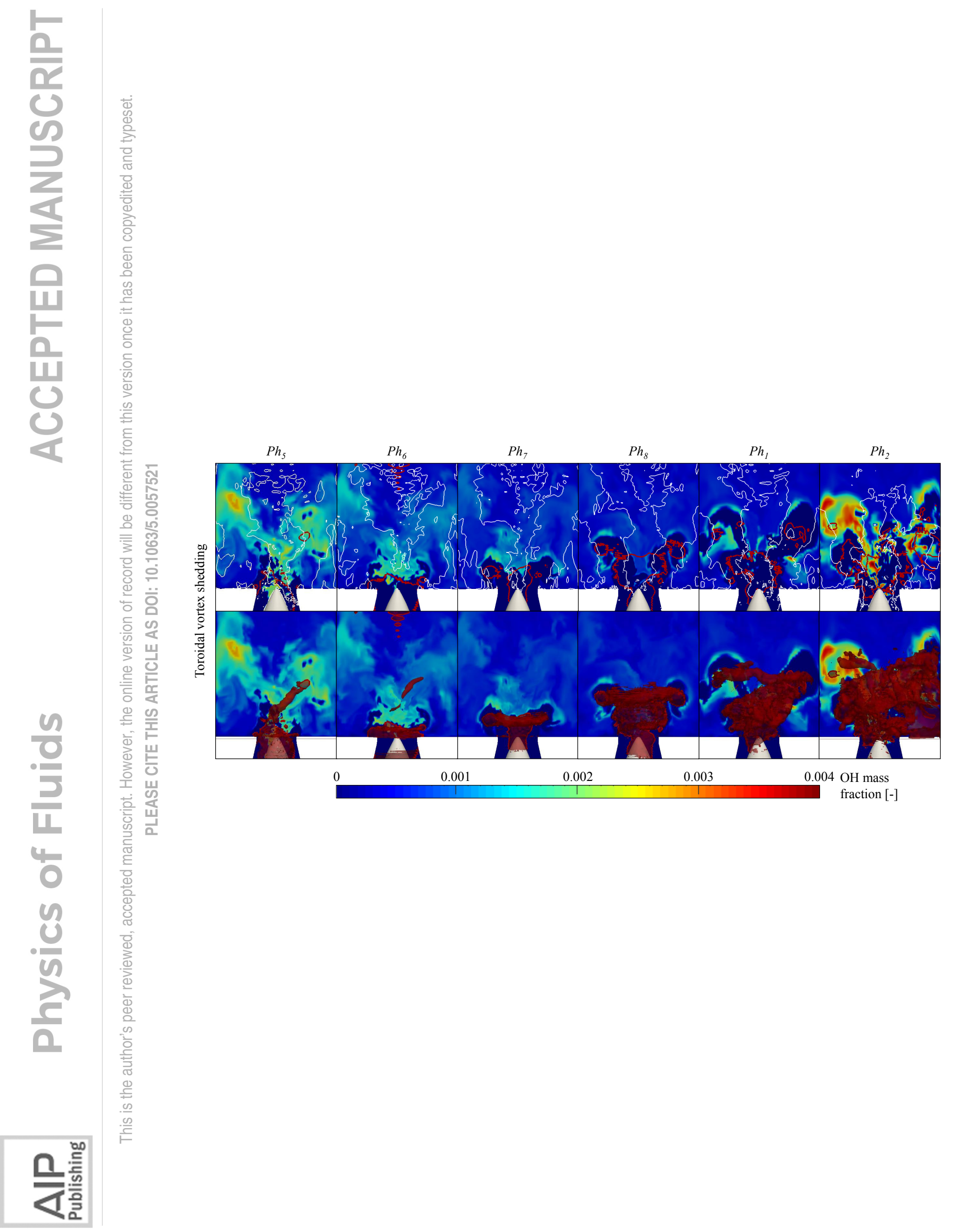




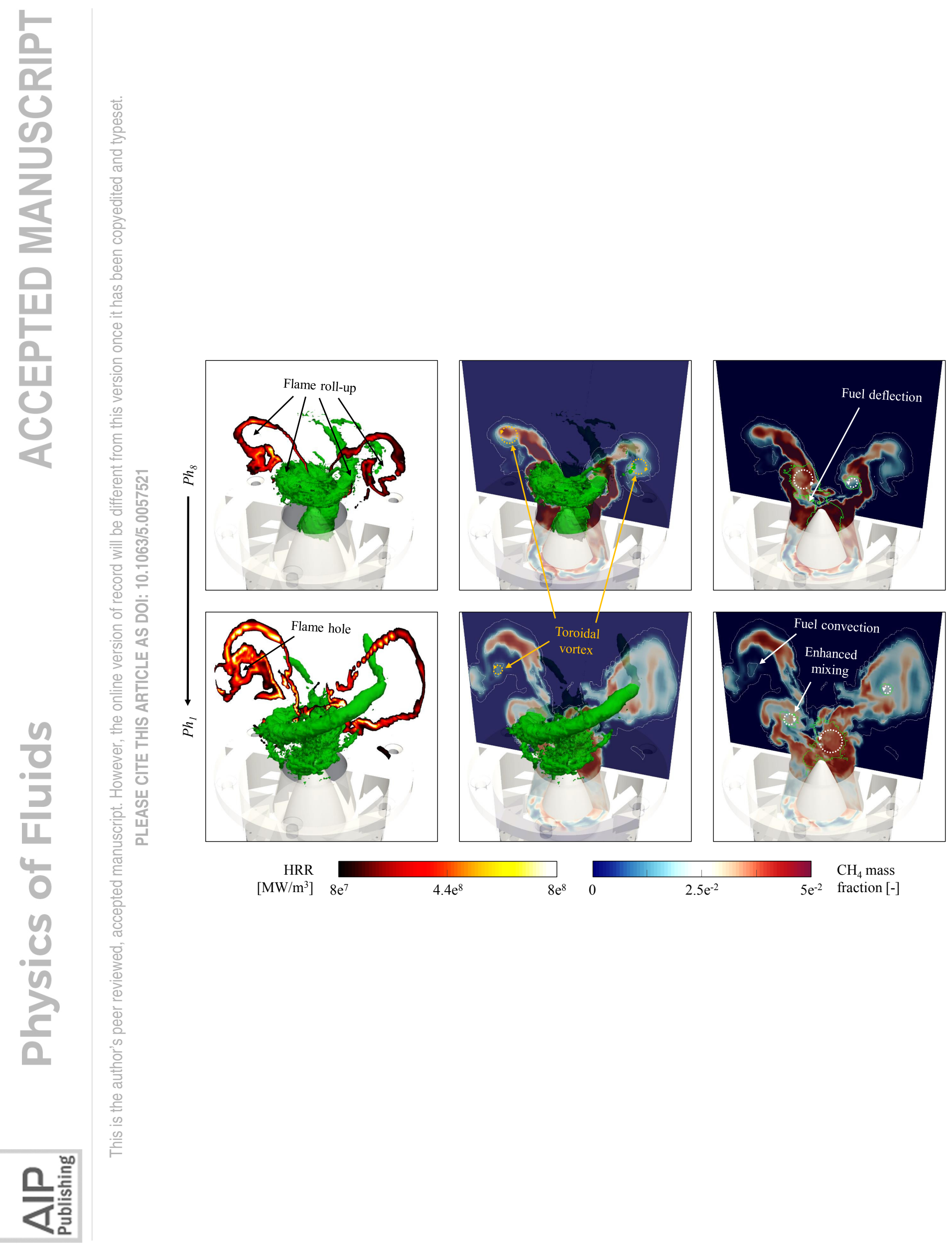



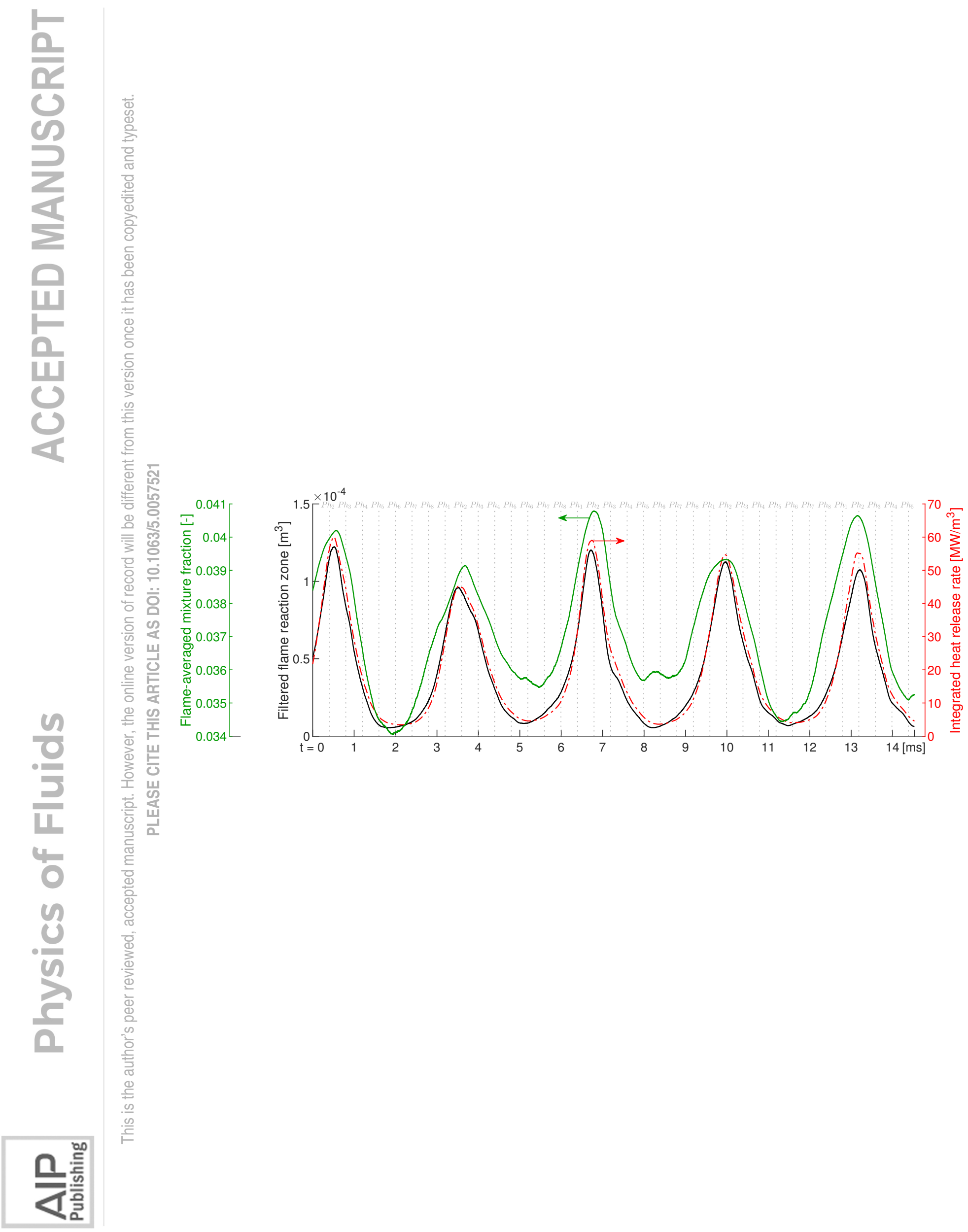


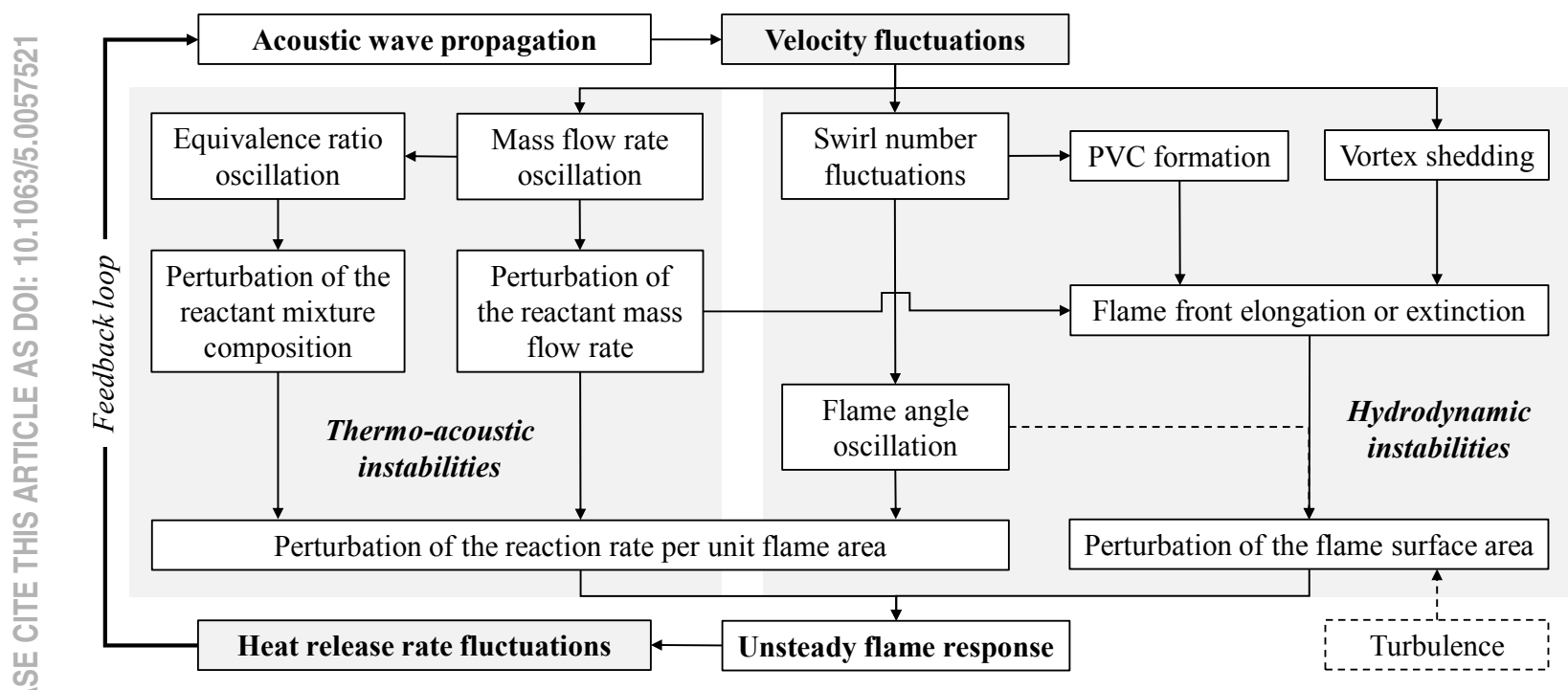

